\title{
Cardy Condition for Open-Closed Field Algebras
}

\author{
Liang Kong ${ }^{1,2,3}$ \\ ${ }^{1}$ Max-Planck-Institute for Mathematics in the Sciences, Inselstrasse 22, D-04103 Leipzig, Germany \\ 2 Institut Des Hautes Études Scientifiques, Le Bois-Marie, 35, Route De Chartres, \\ F-91440 Bures-sur-Yvette, France \\ 3 Max-Planck-Institute for Mathematics, Vivatsgasse 7, D-23111 Bonn, Germany. \\ E-mail: kong@mpim-bonn.mpg.de
}

Received: 10 April 2007 / Accepted: 1 April 2008

Published online: 24 July 2008 - (C) Springer-Verlag 2008

\begin{abstract}
Let $V$ be a vertex operator algebra satisfying certain reductivity and finiteness conditions such that $\mathcal{C}_{V}$, the category of $V$-modules, is a modular tensor category. We study open-closed field algebras over $V$ equipped with nondegenerate invariant bilinear forms for both open and closed sectors. We show that they give algebras over a certain $\mathbb{C}$-extension of the so-called Swiss-cheese partial dioperad, and we can obtain Ishibashi states easily in such algebras. The Cardy condition can be formulated as an additional condition on such open-closed field algebras in terms of the action of the modular transformation $S: \tau \mapsto-\frac{1}{\tau}$ on the space of intertwining operators of $V$. We then derive a graphical representation of $S$ in the modular tensor category $\mathcal{C}_{V}$. This result enables us to give a categorical formulation of the Cardy condition and the modular invariance condition for 1-point correlation functions on the torus. Then we incorporate these two conditions and the axioms of the open-closed field algebra over $V$ equipped with nondegenerate invariant bilinear forms into a tensor-categorical notion called the Cardy $\mathcal{C}_{V} \mid \mathcal{C}_{V \otimes V}$-algebra. In the end, we give a categorical construction of the Cardy $\mathcal{C}_{V} \mid \mathcal{C}_{V \otimes V}$-algebra in the Cardy case.
\end{abstract}

\section{Contents}

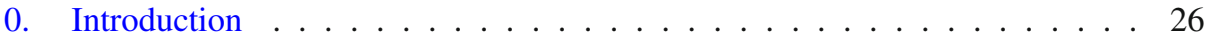

1. Partial Dioperads . . . . . . . . . . . . . . . . . . . . . . . . . . . . . . . .

1.1 Partial dioperads . . . . . . . . . . . . . . . . . . . 31

1.2 Conformal full field algebras . . . . . . . . . . . . . . . . . . 35

1.3 Open-string vertex operator algebras . . . . . . . . . . . . . . . . . . . . . 37

2. Swiss-Cheese Partial Dioperad . . . . . . . . . . . . . . . . 38

2.12 -colored (partial) dioperads . . . . . . . . . . . . . . . 38

2.2 Swiss-cheese partial dioperads . . . . . . . . . . . . . . . . . . . . . . 41

2.3 Open-closed field algebras over $V \ldots \ldots$. . . . . . . . . . . . . . . . . . . . 43

2.4 Ishibashi states . . . . . . . . . . . . . . . . . . . . 48 


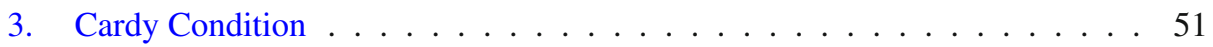

3.1 The first version . . . . . . . . . . . . . . . . 51

3.2 The second version . . . . . . . . . . . . . . . . . 56

4. Modular Tensor Categories . . . . . . . . . . . . . . . . 64

4.1 Preliminaries . . . . . . . . . . . . . . . . 65

4.2 Graphical representation of $S: \tau \mapsto-\frac{1}{\tau} \ldots \ldots \ldots 6$

5. Categorical Formulations and Constructions . . . . . . . . . . . 76

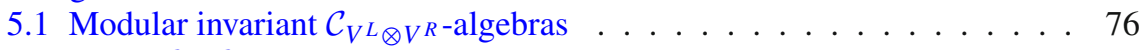

5.2 Cardy $\mathcal{C}_{V} \mid \mathcal{C}_{V \otimes V}$-algebras . . . . . . . . . . . . . . . . . . 80

5.3 Constructions . . . . . . . . . . . . . . . . . . 84

A. The Proof of Lemma $4.30 \ldots \ldots$. . . . . . . . . . . . . . 88

\section{Introduction}

This work is a continuation of the works [HKo1-HKo3,Ko1,Ko2] and a part of an openstring extension of a program on the closed conformal field theory via the theory of vertex operator algebra. This program was initiated by I. Frenkel and largely developed by Huang [H1]-[H11]. Zhu's work [Z] is also very influential in this development.

Segal defined the (closed) conformal field theory [Se1] as a projective monoidal functor from the category of finite ordered sets with morphisms being the conformal equivalent classes of Riemann surfaces with parametrized boundaries to the category of locally convex complete topological vector spaces. This definition is very difficult to work with directly. Taking advantage of the theory of vertex operator algebra, Huang suggested to first construct all necessary structures on a dense subspace of the relevant complete topological vector space [H3], then complete it properly later [H5,H6]. This idea guided us in all our previous works [HKo1-HKo3,Ko1,Ko2], in particular in our formulation of the notion of algebra over the Swiss-cheese partial operad which catches only some genus-zero information of the whole structure on the dense subspace. This seemingly temporary structure on the dense subspace does not necessarily follow from that on the complete topological vector space, thus has its own independent values and is worthwhile to be formulated properly and extended to a theory of all genus. We will call such a theory on the dense subspace a (closed) partial conformal field theory.

More precisely, a partial conformal field theory is a projective monoidal functor $\mathcal{F}: \mathcal{R S} \rightarrow \mathcal{G V}$ between two partial categories $\mathcal{R S}, \mathcal{G} \mathcal{V}$ in which the compositions of morphisms are not always well-defined. $\mathcal{R} \mathcal{S}$ is the category of finite ordered sets, and $\operatorname{Mor}_{\mathcal{R} S}\left(S_{1}, S_{2}\right)$ for any pair of such sets $S_{1}, S_{2}$ is the set of the conformal equivalent classes of closed Riemann surfaces with $\left|S_{1}\right|$ positively oriented punctures and local coordinates and $\left|S_{2}\right|$ negatively oriented punctures and local coordinates [H3], and the compositions of morphisms in $\mathcal{R} \mathcal{S}$ are given by the sewing operations on oppositely oriented punctures ([H3]), where the sewing operations are only partially defined. $\mathcal{G} \mathcal{V}$ is the category of graded vector spaces with finite dimensional homogeneous spaces and a weak topology induced from the restricted dual spaces. For any pair of $A, B \in \mathrm{Ob}(\mathcal{G} \mathcal{V})$, $\operatorname{Mor}_{\mathcal{G V}}(A, B)$ is the set of continuous linear maps from $A$ to $\bar{B}:=\prod_{n \in G_{B}} B_{(n)}$, where the abelian group $G_{B}$ gives the grading on $B$. For any pair of morphisms $A \stackrel{g}{\rightarrow} \bar{B}$ and $B \stackrel{f}{\rightarrow} \bar{C}$ in $\mathcal{G} \mathcal{V}$, using the projector $P_{n}: \bar{B} \rightarrow B_{(n)}$, we define $f \circ g(u):=$ $\sum_{n \in G_{B}} f\left(P_{n} g(u)\right), \forall u \in A$, and $f \circ g$ is well-defined only when the sum is absolutely convergent for all $u \in A$. We remark that by replacing the surfaces with parametrized boundaries in Segal's definition by the surfaces with oriented punctures and local coor- 
dinates in $\mathcal{R S}$ we have enlarged the morphism sets. Thus a Segal's functor may not be extendable to a functor on $\mathcal{R S}$.

The above definition can be easily extended to include open strings by adding Riemann surfaces with (unparametrized!) boundaries and both oriented interior punctures and punctures on the boundaries to $\mathcal{R} \mathcal{S}$. We will call this open-string extended theory an open-closed partial conformal field theory. We denote the graded vector spaces associated to interior punctures (closed strings at infinity) and boundary punctures (open strings at infinity) by $V_{c l}$ and $V_{o p}$ respectively.

The sets of all genus-zero closed surfaces (spheres) with an arbitrary number of positively and negatively oriented punctures form a structure of sphere partial dioperad $\mathbb{K}$ (see the definition in Sect. 1.1). It includes as a substructure the sphere partial operad [H3], which only includes spheres with a single negatively oriented puncture. The sphere partial dioperad allows all sewing operations as long as the surfaces after sewing are still genus-zero. Hence to construct genus-zero partial conformal field theory amounts to construct projective $\mathbb{K}$-algebras or algebras over a certain extension of $\mathbb{K}$. In [HKo2], we introduced the notion of a conformal full field algebra over $V^{L} \otimes V^{R}$ equipped with a nondegenerate invariant bilinear form, where $V^{L}$ and $V^{R}$ are two vertex operator algebras of central charge $c^{L}$ and $c^{R}$ respectively and satisfy certain finiteness and reductivity conditions. Theorem 2.7 in [Kol] can be reformulated as follows: a conformal full field algebra $V_{c l}$ over $V^{L} \otimes V^{R}$ equipped with a nondegenerate invariant bilinear form canonically gives on $V_{c l}$ an algebra over $\tilde{\mathbb{K}}^{c^{L}} \otimes \overline{\tilde{\mathbb{K}}^{c^{R}}}$, which is a partial dioperad extension of $\mathbb{K}$.

The sets of all genus-zero surfaces with one (unparametrized!) boundary component (disks) and an arbitrary number of oriented boundary punctures form the so-called disk partial dioperad denoted by $\mathbb{D}$ (see the definition in Sect. 1.1). $\mathcal{F}$ restricted on $\mathbb{D}$ induces a structure of algebra over a certain extension of $\mathbb{D}$ on $V_{o p}$. In [HKo1], Huang and I introduced the notion of an open-string vertex operator algebra. We will show in Sect. 1.3 that an open-string vertex operator algebra of central charge $c$ equipped with a nondegenerate invariant bilinear form canonically gives an algebra over $\tilde{\mathbb{D}}^{c}$, which is a partial dioperad extension of $\mathbb{D}$.

The sets of all genus-zero surfaces with only one (unparametrized!) boundary component and arbitrary number of oriented interior punctures and boundary punctures form the so-called Swiss-Cheese partial dioperad $\mathbb{S}$ (see the definition in Sect. 2.2). A typical elements in $\mathbb{S}$ is depicted in Fig. 1, where boundary punctures are drawn as an infinitely long strip (or an open string) and interior punctures are drawn as an infinitely long tube (or a closed string). Let $V$ be a vertex operator algebra of central charge $c$ satisfying the conditions in Theorem 0.1. We will show in Sect. 2.3 that an open-closed field algebra over $V$, which contains an open-string vertex operator algebra $V_{o p}$ and a conformal full field algebra $V_{c l}$, satisfying a $V$-invariant boundary condition [Ko2] and equipped with nondegenerate invariant bilinear forms on both $V_{o p}$ and $V_{c l}$, canonically gives an algebra over $\tilde{\mathbb{S}}^{c}$, which is an extension of $\mathbb{S}$.

Note that all surfaces of any genus with an arbitrary number of boundary components, interior punctures and boundary punctures can be obtained by applying sewing operations to elements in $\mathbb{S}$. Therefore, except for some compatibility conditions coming from different decompositions of the same surfaces with higher genus, an algebra over $\tilde{\mathbb{S}}^{c}$ if extendable uniquely determine the entire theory of all genus. In particular, the famous Ishibashi states [I] can be obtained in an open-closed field algebra equipped with nondegenerate invariant bilinear forms. An Ishibashi state is a coherent state $\psi$ 




Fig. 1. A typical element in the Swiss-Cheese partial dioperad $\mathbb{S}$

in $\overline{V_{c l}}$ such that $\left(L^{L}(n)-L^{R}(-n)\right) \psi=0, \forall n \in \mathbb{Z}$. In physics, Ishibashi states are obtained by solving the above equation. In Sect. 2.4, we show how to obtain Ishibashi states constructively and geometrically from vacuum-like states in $V_{o p}$.

Other surfaces which are not included in $\mathbb{S}$ only provide additional compatibility conditions. In 2-d topological field theories, only three additional compatibility conditions are needed to ensure the consistency of a theory of all genus [La, Mo1, Se2, MSeg, AN, LP]. The first compatibility condition says that both $V_{o p}$ and $V_{c l}$ are finite dimensional. This guarantees the convergence of all higher genus correlation functions. The second condition is the modular invariance condition for 1-point correlation functions on torus. It is due to two different decompositions of the same torus as depicted in Fig. 2. This condition is automatically satisfied in 2-d topological field theories, but nontrivial in conformal field theories. The third condition is the famous Cardy condition which is again due to two different decompositions of a single surface as shown in Fig. 3.

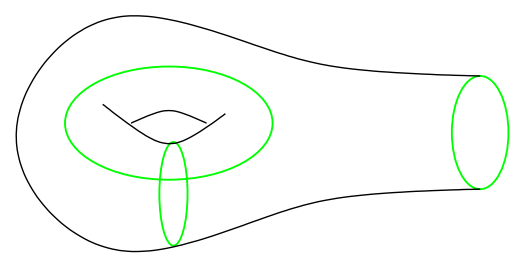

Fig. 2. Modular invariance condition for 1-pt correlation functions on torus

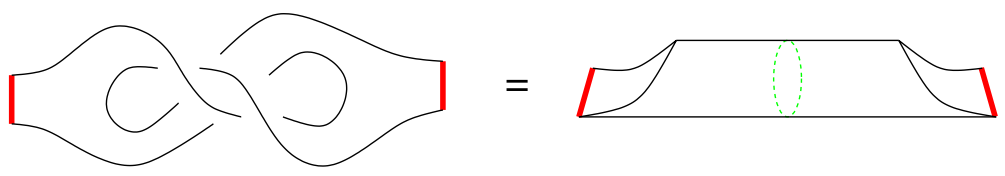

Fig. 3. Cardy condition 
Now we turn to the compatibility conditions in conformal field theory. In this case, both $V_{c l}$ and $V_{o p}$ in any nontrivial theory are infinite dimensional. We need to require the convergence of all correlation functions of all genus. This is a highly nontrivial condition and not easy to check for examples. So far the only known convergence results are in genus-zero [H7] and genus-one theories [Z,DLM,Mi1,Mi2,H9]. We recall a theorem by Huang.

Theorem 0.1 ([H10,H11]). If $(V, Y, \mathbf{1}, \omega)$ is a simple vertex operator algebra $V$ satisfying the following conditions: 1 . $V$ is $C_{2}$-cofinite; $2 . V_{n}=0$ for $n<0, V_{(0)}=\mathbb{C} \mathbf{1}$, $V^{\prime}$ is isomorphic to $V$ as $V$-module; 3. all $\mathbb{N}$-gradable weak $V$-modules are completely reducible, then the direct sum of all inequivalent irreducible $V$-modules has a natural structure of intertwining operator algebra [H4], and the category $\mathcal{C}_{V}$ of $V$-modules has a structure of vertex tensor category [HL1]-[HL4,H2] and modular tensor category $[R T, T]$.

For the intertwining operator algebra given in Theorem 0.1 , Huang also proved in $[\mathrm{H} 7, \mathrm{H} 9]$ that the products of intertwining operators and their $q$-traces have a certain nice convergence and analytic extension properties. These properties are sufficient for the construction of genus-zero and genus-one correlation functions in closed partial conformal field theory [HKo2,HKo3]. Since the modular tensor category $\mathcal{C}_{V}$ supports an action of mapping class groups of all genus, it is reasonable to believe that the above conditions on $V$ are also sufficient to guarantee the convergence of correlation functions of all genus.

Assumption 0.2. In this work, we fix a vertex operator algebra $V$ with central charge $c$, which is assumed to satisfy the conditions in Theorem 0.1 without further announcement.

Besides the convergence condition and the axioms of projective $\mathbb{K}$-algebra, Sonoda [So] argued on a physical level of rigor that it is sufficient to check the modular invariance condition in Fig. 2 in order to have a consistent partial conformal field theory of all genus. This modular invariance condition was studied in [HKo3]. In the framework of conformal full field algebra over $V^{L} \otimes V^{R}$, it was formulated algebraically as a modular invariance property of an intertwining operator of $V^{L} \otimes V^{R}$.

For an open-closed theory, besides the convergence condition, the axioms of algebra over $\tilde{\mathbb{S}}^{c}$ and the modular invariance condition, Lewellen [Le] argued on a physical level of rigor that the only remaining compatibility condition one needs is the Cardy condition. The Cardy condition in an open-closed conformal field theory is more complicated than that in topological theory and has never been fully written down by physicists. In Sect. 3.1, we derive the Cardy condition from the axioms of open-closed partial conformal field theory by writing out two sides of the Cardy condition in Figure 3 explicitly in terms of the ingredients of an open-closed field algebra (see Definition 3.4). Using results in $[\mathrm{H} 9, \mathrm{H} 10]$, we can show that the Cardy condition can be reformulated as an invariance condition of the modular transformation $S: \tau \mapsto-\frac{1}{\tau}$ on intertwining operators.

There are still more compatibility conditions which were not discussed in [So,Le]. One also need to prove a certain algebraic version of uniformization theorems (see $[\mathrm{H} 1, \mathrm{H} 3]$ for the genus-zero case). Such results for genus larger than 0 are still not available. But it seems that no additional assumption on $V$ is needed. They should follow automatically from the properties of the Virasoro algebra and intertwining operators. This uniformization problem and convergence problems are not pursued further in this work. 
In order to take advantage of some powerful tools, such as the graphic calculus in tensor category, in the study of the modular invariance condition and the Cardy condition, we would like to obtain categorical formulations of these conditions. It requires us to know the action of the modular transformation $S$ in $\mathcal{C}_{V}$. Although the action of $S L(2, \mathbb{Z})$ in a modular tensor category is explicitly known [MSei3, V, Ly, Ki, BK2], its relation to the modular transformation of the $q$-trace of the products of intertwining operators of $V$ is not completely clear. This relation was first suggested by I. Frenkel and studied by Moore and Seiberg in [MSei3] but only on a physical level of rigor. Using Huang's results on the modular tensor category $[\mathrm{H} 10, \mathrm{H} 11]$, we derive a graphical representation of $S$ in $\mathcal{C}_{V}$. This result enables us to give categorical formulations of the modular invariance condition and the Cardy condition. We incorporate them with the categorical formulation of open-closed field algebra over $V$ equipped with nondegenerate invariant bilinear forms into a tensor-categorical notion called the $\operatorname{Cardy} \mathcal{C}_{V} \mid \mathcal{C}_{V \otimes V}$-algebra. As we discussed in previous paragraphs, it is reasonable to believe that open-closed partial conformal field theories of all genus satisfying the $V$-invariant boundary condition [Ko2] are classified by Cardy $\mathcal{C}_{V} \mid \mathcal{C}_{V \otimes V}$-algebras. However, to construct the high-genus theories explicitly is still a hard open problem which is not pursued in this work. In the end, we give an explicit construction of Cardy the $\mathcal{C}_{V} \mid \mathcal{C}_{V \otimes V}$-algebra in the so-called Cardy case in the physics literature (see for example [FFRS]).

Note that this work is somewhat complementary to the works of Fjelstad, Fuchs, Runkel, Schweigert [FS,FRS1-FRS4,FjFRS1,FjFRS2]. We will leave a detailed study of the relationship between these two approaches to [KR].

The layout of this work is as follows: in Sect. 1, we introduce the notion of a sphere partial dioperad and disk partial dioperad and study algebras over them; in Sect. 2, we introduce the notions of the Swiss-cheese partial dioperad $\mathbb{S}$ and its $\mathbb{C}$-extension $\tilde{\mathbb{S}}^{c}$, and show that an open-closed conformal field algebra over $V$ equipped with nondegenerate invariant bilinear forms canonically gives an algebra over $\tilde{\mathbb{S}}^{c}$, and we also construct Ishibashi states in such algebras; in Sect. 3, we give two formulations of the Cardy condition; in Sect. 4, we derive a graphic representation of the modular transformation $S$; in Sect. 5, we give the categorical formulations of the nondegenerate invariant bilinear forms, the modular invariance condition and the Cardy condition. Then we introduce the notion of the Cardy $\mathcal{C}_{V} \mid \mathcal{C}_{V \otimes V}$-algebra and give a construction.

Convention of notations: $\mathbb{N}, \mathbb{Z}, \mathbb{Z}_{+}, \mathbb{R}, \mathbb{R}_{+}, \mathbb{C}$ denote the set of natural numbers, integers, positive integers, real numbers, positive real numbers, complex numbers, respectively. Let $\mathbb{H}=\{z \in \mathbb{C} \mid \operatorname{Im} z>0\}$ and $\overline{\mathbb{H}}=\{z \in \mathbb{C} \mid \operatorname{Im} z<0\}$. Let $\hat{\mathbb{R}}, \hat{\mathbb{C}}$ and $\hat{\mathbb{H}}$ be the one point compactification of real line, complex plane and up-half plane (including the $\mathbb{R}$-boundary) respectively. Let $\mathbb{R}_{+}$and $\mathbb{C}^{\times}$be the multiplication groups of positive real and nonzero complex numbers respectively. The ground field is always chosen to be $\mathbb{C}$.

Throughout this work, we choose a branch cut for logarithm as follows:

$$
\log z=\log |z|+i \operatorname{Arg} z, \quad 0 \leq \operatorname{Arg} z<2 \pi .
$$

We define power functions of two different types of complex variables as follows:

$$
z^{s}:=e^{s \log z}, \quad \bar{z}^{s}:=e^{s \overline{\log z}}, \quad \forall s \in \mathbb{R} .
$$

\section{Partial Dioperads}

In Sect. 1.1, we recall the definition of (partial) dioperad and algebra over it, and introduce sphere partial dioperad $\mathbb{K}$, disk partial dioperad $\mathbb{D}$ and their extensions $\tilde{\mathbb{K}}^{c^{L}} \otimes \overline{\tilde{\mathbb{K}}^{c^{R}}}$, 
$\tilde{\mathbb{D}}^{c}$ as examples. In Sect. 1.2, we discuss an algebra over $\tilde{\mathbb{K}}^{c^{L}} \otimes \overline{\tilde{\mathbb{K}}^{c^{R}}}$ from a conformal full field algebra over $V^{L} \otimes V^{R}$. In Sect. 1.3, we discuss an algebra over $\tilde{D}^{c}$ from an open-string vertex operator algebra.

1.1. Partial dioperads. Let us first recall the definition of dioperad given by Gan [G]. Let $S_{n}$ be the automorphism group of the set $\{1, \ldots, n\}$ for $n \in \mathbb{Z}_{+}$. Let $m=m_{1}+\cdots+m_{n}$ be an ordered partition and $\sigma \in S_{n}$. The block permutation $\sigma_{\left(m_{1}, \ldots, m_{n}\right)} \in S_{m}$ is the permutation which permutes $n$ intervals of lengths $m_{1}, \ldots, m_{n}$ in the same way as $\sigma$ permutes $1, \ldots, n$. Let $\sigma_{i} \in S_{m_{i}}, i=1, \ldots, k$. We view the element $\left(\sigma_{1}, \ldots, \sigma_{k}\right) \in S_{m_{1}} \times \cdots \times S_{m_{k}}$ naturally as an element in $S_{m}$ by the canonical embedding $S_{m_{1}} \times \cdots \times S_{m_{k}} \hookrightarrow S_{m}$. For any $\sigma \in S_{n}$ and $1 \leq i \leq n$, we define a map $\hat{i}:\{1, \ldots, n-1\} \rightarrow\{1, \ldots, n\}$ by $\hat{i}(j)=j$ if $j<i$ and $\hat{i}(j)=j+1$ if $j \geq i$ and an element $\hat{i}(\sigma) \in S_{n-1}$ by

$$
\hat{i}(\sigma)(j):=\hat{i}^{-1} \circ \sigma \circ \widehat{\sigma^{-1}(i)}(j) .
$$

Definition 1.1. A dioperad consists of a family of sets $\{\mathcal{P}(m, n)\}_{m, n \in \mathbb{N}}$ with an action of $S_{m} \times S_{n}$ on $\mathcal{P}(m, n)$ for each pair of $m, n \in \mathbb{Z}_{+}$, a distinguished element $I_{\mathcal{P}} \in \mathcal{P}(1,1)$ and substitution maps

$$
\begin{aligned}
& \mathcal{P}(m, n) \times \mathcal{P}\left(k_{1}, l_{1}\right) \times \cdots \times \mathcal{P}\left(k_{n}, l_{n}\right) \stackrel{\gamma_{\left(i_{1}, \ldots, i_{n}\right)}}{\longrightarrow} \mathcal{P}\left(m-n+k_{1} \cdots+k_{n}, l_{1}+\cdots+l_{n}\right) \\
&\left(P, P_{1}, \ldots, P_{n}\right) \mapsto \quad \gamma_{\left(i_{1}, \ldots, i_{n}\right)}\left(P ; P_{1}, \ldots, P_{n}\right)
\end{aligned}
$$

for $m, n, l_{1}, \ldots, l_{n} \in \mathbb{N}, k_{1}, \ldots, k_{n} \in \mathbb{Z}_{+}$and $1 \leq i_{j} \leq k_{j}, j=1, \ldots, n$, satisfying the following axioms:

1. Unit properties: For $P \in \mathcal{P}(m, n)$,

(a) left unit property: $\gamma_{(i)}\left(I_{\mathcal{P}} ; P\right)=P$ for $1 \leq i \leq m$,

(b) right unit property: $\gamma_{(1, \ldots, 1)}\left(P ; I_{\mathcal{P}}, \ldots, I_{\mathcal{P}}\right)=P$.

2. Associativity: for $P \in \mathcal{P}(m, n), Q_{i} \in \mathcal{P}\left(k_{i}, l_{i}\right), i=1, \ldots, n, R_{j} \in \mathcal{P}\left(s_{j}, t_{j}\right)$, $j=1, \ldots, l=l_{1}+\cdots+l_{n}$, we have

$$
\gamma_{\left(q_{1}, \ldots, q_{l}\right)}\left(\gamma_{\left(p_{1}, \ldots, p_{n}\right)}\left(P ; Q_{1}, \ldots, Q_{n}\right) ; R_{1}, \ldots, R_{l}\right)=\gamma_{\left(p_{1}, \ldots, p_{n}\right)}\left(P ; P_{1}, \ldots, P_{n}\right)
$$

where $P_{i}=\gamma_{\left(q_{l_{1}+\cdots+l_{i-1}+1}, \ldots, q_{l_{1}+\cdots+l_{i}}\right)}\left(Q_{i} ; R_{l_{1}+\cdots+l_{i-1}+1}, \ldots, R_{l_{1}+\cdots+l_{i}}\right)$ for $i=1, \ldots, n$.

3. Permutation property: For $P \in \mathcal{P}(m, n), Q_{i} \in P\left(k_{i}, l_{i}\right), i=1, \ldots, n,(\sigma, \tau) \in$ $S_{m} \times S_{n},\left(\sigma_{i}, \tau_{i}\right) \in S_{k_{i}} \times S_{l_{i}}, i=1, \ldots, n$,

$$
\begin{aligned}
& \gamma_{\left(i_{1}, \ldots, i_{n}\right)}\left((\sigma, \tau)(P) ; Q_{1}, \ldots, Q_{n}\right) \\
& \quad=\left(\left(\sigma, \tau_{\left(k_{1}-1, \ldots, k_{n}-1\right)}\right), \tau_{\left(l_{1}, \ldots, l_{n}\right)}\right) \gamma_{\left(i_{\tau(1)}, \ldots, i_{\tau(n)}\right)}\left(P ; Q_{\tau(1)}, \ldots, Q_{\tau(n)}\right), \\
& \gamma_{\left(i_{1}, \ldots, i_{n}\right)}\left(P ;\left(\sigma_{1}, \tau_{1}\right)\left(Q_{1}\right), \ldots,\left(\sigma_{n}, \tau_{n}\right)\left(Q_{n}\right)\right) \\
& \quad=\left(\left(\mathrm{id}, \hat{i}_{1}\left(\sigma_{1}\right), \ldots, \hat{i}_{n}\left(\sigma_{n}\right)\right),\left(\tau_{1}, \ldots, \tau_{n}\right)\right) \gamma_{\left(\sigma_{1}^{-1}\left(i_{1}\right), \ldots, \sigma_{n}^{-1}\left(i_{n}\right)\right)}\left(P ; Q_{1}, \ldots, Q_{n}\right) .
\end{aligned}
$$

We denote such a dioperad as $\left(\mathcal{P}, \gamma_{\mathcal{P}}, I_{\mathcal{P}}\right)$ or simply $\mathcal{P}$.

Remark 1.2. We define compositions $i{ }_{i}$ as follows:

$$
P_{i} \circ_{j} Q:=\gamma_{(1, \ldots, 1, j, 1, \ldots 1)}\left(P ; I_{\mathcal{P}}, \ldots, I_{\mathcal{P}}, Q, I_{\mathcal{P}}, \ldots, I_{\mathcal{P}}\right) .
$$

It is easy to see that $\gamma_{\left(i_{1}, \ldots, i_{n}\right)}$ can be reobtained from ${ }_{i} \circ_{j}$. In $[\mathrm{G}]$, the definition of dioperad is given in terms of $i 0_{j}$ instead of $\gamma_{\left(i_{1}, \ldots, i_{n}\right)}$. 
Definition 1.3. A partial dioperad has a similar definition as that of dioperad except the map $\gamma_{\left(i_{1}, \ldots, i_{n}\right)}$ or $i_{i} \circ_{j}$ is only partially defined and the same associativity holds whenever both sides of (1.2) exist. A (partial) nonassociative dioperad consists of the same data as those of a (partial) dioperad satisfying all the axioms of a (partial) dioperad except the associativity.

The notion of homomorphism and isomorphism of a (partial pseudo-) dioperad are naturally defined.

Remark 1.4. In the case of a partial dioperad, the definition using $\gamma_{\left(i_{1}, \ldots, i_{n}\right)}$ or ${ }_{i} \circ_{j}$ may have subtle differences in the domains on which $\gamma_{\left(i_{1}, \ldots, i_{n}\right)}$ or $i \circ_{j}$ is defined (see Appen$\operatorname{dix} C$ in [H2] for more details). These differences have no effect on those algebras over partial dioperads considered in this work. So we will simply ignore these differences.

Remark 1.5. Notice that a (partial nonassociative) dioperad $\{\mathcal{P}(m, n)\}_{m, n \in \mathbb{N}}$ naturally contains a (partial nonassociative) operad $\{\mathcal{P}(1, n)\}_{n \in \mathbb{N}}$ as a substructure.

Definition 1.6. A subset $G$ of $\mathcal{P}(1,1)$ is called a rescaling group of $\mathcal{P}$ if the following conditions are satisfied:

1. For any $g, g_{1}, \ldots, g_{n} \in G, Q \in P(m, n), \gamma_{(i)}(g ; Q)$ and $\gamma_{(1, \ldots, 1)}\left(Q ; g_{1}, \ldots, g_{n}\right)$ are always well-defined for $1 \leq i \leq m$.

2. $I_{\mathcal{P}} \in G$ and $G$ together with the identity element $I_{\mathcal{P}}$ and multiplication map $\gamma_{(1)}$ : $G \times G \rightarrow G$ is a group.

Definition 1.7. A $G$-rescalable partial dioperad is a partial dioperad $\mathcal{P}$ such that for any $P \in \mathcal{P}(m, n), Q_{i} \in \mathcal{P}\left(k_{i}, l_{i}\right), i=1, \ldots, n$ there exist $g_{i} \in G, i=1, \ldots, n$ such that $\gamma_{\left(i_{1}, \ldots, i_{n}\right)}\left(P ; \gamma_{\left(i_{1}\right)}\left(g_{1} ; Q_{1}\right), \ldots, \gamma_{\left(i_{n}\right)}\left(g_{n} ; Q_{n}\right)\right)$ is well-defined.

The first example of partial dioperad in which we are interested in this work comes from $\mathbb{K}=\left\{\mathbb{K}\left(n_{-}, n_{+}\right)\right\}_{n_{-}, n_{+} \in \mathbb{N}}[\mathrm{Ko} 2]$, a natural extension of the sphere partial operad $K$ [H3]. More precisely, $\mathbb{K}\left(n_{-}, n_{+}\right)$is the set of the conformal equivalent classes of a sphere with $n_{-}\left(n_{+}\right)$ordered negatively (positively) oriented punctures and local coordinate map around each puncture. In particular, $\mathbb{K}(0,0)$ is an one-element set consisting of the conformal equivalent class of a sphere with no additional structure. We simply denote this element as $\hat{\mathbb{C}}$. We use

$$
\begin{aligned}
Q=\left(\left(z_{-1} ; a_{0}^{(-1)}, A^{(-1)}\right)\right. & , \ldots,\left(z_{-n_{-}} ; a_{0}^{\left(-n_{-}\right)}, A^{\left(-n_{-}\right)}\right) \mid \\
& \left.\left(z_{1} ; a_{0}^{(1)}, A^{(1)}\right) \ldots\left(z_{n_{+}} ; a_{0}^{\left(n_{+}\right)}, A^{\left(n_{+}\right)}\right)\right)_{\mathbb{K}},
\end{aligned}
$$

where $z_{i} \in \hat{\mathbb{C}}, a_{0}^{(i)} \in \mathbb{C}^{\times}, A^{(i)} \in \mathbb{C}^{\infty}$ for $i=-n_{-}, \ldots,-1,1, \ldots, n_{+}$, to denote a sphere $\hat{\mathbb{C}}$ with positively (negatively) oriented punctures at $z_{i} \in \hat{\mathbb{C}}$ for $i=1, \ldots, n_{+}$ $\left(i=-1, \ldots,-n_{-}\right)$, and with local coordinate map $f_{i}$ around each puncture $z_{i}$ given by:

$$
\begin{aligned}
f_{i}(w) & =\left.e^{\sum_{j=1}^{\infty} A_{j}^{(i)} x^{j+1} \frac{d}{d x}}\left(a_{0}^{(i)}\right)^{x} \frac{d}{d x} x\right|_{x=w-z_{i}} & & \text { if } z_{i} \in \mathbb{C}, \\
& =\left.e^{\sum_{j=1}^{\infty} A_{j}^{(i)} x^{j+1} \frac{d}{d x}}\left(a_{0}^{(i)}\right)^{x} \frac{d}{d x} x\right|_{x=\frac{-1}{w}} & & \text { if } z_{i}=\infty .
\end{aligned}
$$


We introduce a useful notation $\bar{Q}$ defined as follows:

$$
\begin{aligned}
\bar{Q}=\left(\left(\bar{z}_{-1} ; \overline{a_{0}^{(-1)}}, \overline{A^{(-1)}}\right)\right. & , \ldots,\left(\bar{z}_{-n_{-}} ; \overline{a_{0}^{\left(-n_{-}\right)}}, \overline{\left.A^{\left(-n_{-}\right)}\right)}\right. \\
& \left.\left(\bar{z}_{1} ; \overline{a_{0}^{(1)}}, \overline{A^{(1)}}\right) \ldots\left(\bar{z}_{n_{+}} ; \overline{a_{0}^{\left(n_{+}\right)}}, \overline{A^{\left(n_{+}\right)}}\right)\right)_{\mathbb{K}},
\end{aligned}
$$

where the "overline" represents complex conjugations.

We denote the set of all such $Q$ as $\mathcal{T}_{\mathbb{K}}\left(n_{-}, n_{+}\right)$. Let $\mathcal{T}_{\mathbb{K}}:=\left\{\mathcal{T}_{\mathbb{K}}\left(n_{-}, n_{+}\right)\right\}_{n_{-}, n_{+} \in \mathbb{N}}$. There is an action of $S L(2, \mathbb{C})$ on $\mathcal{T}_{\mathbb{K}}\left(n_{-}, n_{+}\right)$as Mobius transformations. It is clear that

$$
\mathbb{K}\left(n_{-}, n_{+}\right)=\mathcal{T}_{\mathbb{K}}\left(n_{-}, n_{+}\right) / S L(2, \mathbb{C}) .
$$

We denote the quotient map $\mathcal{T}_{\mathbb{K}} \rightarrow \mathbb{K}$ as $\pi_{\mathbb{K}}$. The identity $I_{\mathbb{K}} \in \mathbb{K}(1,1)$ is given by

$$
I_{\mathbb{K}}=\pi_{\mathbb{K}}((\infty, 1, \mathbf{0}) \mid(0,1, \mathbf{0})),
$$

where $\mathbf{0}=(0,0, \ldots) \in \prod_{n=1}^{\infty} \mathbb{C}$. The composition $i_{j}$ is provided by the sewing operation $i \infty_{-j}$ [H3]. In particular, for $n_{1}, m_{2} \geq 1, P \in \mathbb{K}\left(m_{1}, n_{1}\right)$ and $Q \in \mathbb{K}\left(m_{2}, n_{2}\right)$, $P_{i} \infty_{-j} Q$ is the sphere with punctures obtained by sewing the $i^{\text {th }}$ positively oriented puncture of $P$ with the $j^{\text {th }}$ negatively oriented puncture of $Q$. The $S_{n_{-}} \times S_{n_{+}}$-action on $\mathbb{K}\left(n_{-}, n_{+}\right)$(or $\left.\mathcal{T}\left(n_{-}, n_{+}\right)\right)$is the natural one. Moreover, the set

$$
\left\{((\infty, 1, \mathbf{0}) \mid(0, a, \mathbf{0})) \mid a \in \mathbb{C}^{\times}\right\}
$$

together with multiplication $1 \infty_{1}$ is a group which can be canonically identified with group $\mathbb{C}^{\times}$. It is clear that $\mathbb{K}$ is a $\mathbb{C}^{\times}$-rescalable partial dioperad. We call it sphere partial dioperad.

The $\mathbb{C}$-extensions of $\mathbb{K}$, such as $\mathbb{K}^{c}$ and $\tilde{\mathbb{K}}^{c^{L}} \otimes \overline{\tilde{\mathbb{K}}^{\overline{c^{R}}}}$ for $c, c^{L}, c^{R} \in \mathbb{C}$, are trivial line bundles over $\mathbb{K}$ with natural $\mathbb{C}^{\times}$-rescalable partial dioperad structures (see Sect. 6.8 in [H2]). Moreover, we denote the canonical section $\mathbb{K} \rightarrow \tilde{\mathbb{K}}^{c^{L}} \otimes \overline{\tilde{\mathbb{K}}^{c^{R}}}$ as $\psi_{\mathbb{K}}$.

The next example of partial dioperad is $\mathbb{D}=\left\{\mathbb{D}\left(n_{-}, n_{+}\right)\right\}_{n_{-}, n_{+} \in \mathbb{N}}$, which is an extension of the partial operad of a disk with strips $\Upsilon$ introduced [HKo1]. More precisely, $\mathbb{D}\left(n_{-}, n_{+}\right)$is the set of conformal equivalent classes of disks with ordered puncture on their boundaries and local coordinate map around each puncture. In particular, $\mathbb{D}(0,0)$ is an one-element set consisting of the conformal equivalent class of a disk with no additional structure. We simply denote this element as $\hat{H}$. We use

$$
\begin{aligned}
Q=\left(\left(r_{-n_{-}} ; b_{0}^{\left(-n_{-}\right)}, B^{\left(-n_{-}\right)}\right), \ldots,\left(r_{-1} ; b_{0}^{(-1)}, B^{(-1)}\right) \mid\right. \\
\left.\left(r_{1} ; b_{0}^{(1)}, B^{(1)}\right) \ldots\left(r_{n_{+}} ; b_{0}^{\left(n_{+}\right)}, B^{\left(n_{+}\right)}\right)\right) \mathbb{D},
\end{aligned}
$$

where $r_{i} \in \hat{\mathbb{R}}, a_{0}^{(i)} \in \mathbb{R}_{+}, B^{(i)} \in \mathbb{R}^{\infty}$ for $i=-n_{-}, \ldots,-1,1, \ldots, n_{+}$, to denote a disk $\hat{\mathbb{H}}$ with positively (negatively) oriented punctures at $r_{i} \in \hat{\mathbb{R}}$ for $i=1, \ldots, n_{+}$ $\left(i=-1, \ldots,-n_{-}\right)$, and with local coordinate map $g_{i}$ around each punctures $r_{i}$ given by:

$$
\begin{aligned}
g_{i}(w) & =\left.e^{\sum_{j=1}^{\infty} B_{j}^{(i)} x^{j+1} \frac{d}{d x}}\left(b_{0}^{(i)}\right)^{x} \frac{d}{d x} x\right|_{x=w-r_{i}} & & \text { if } r_{i} \in \mathbb{R}, \\
& =\left.e^{\sum_{j=1}^{\infty} B_{j}^{(i)} x^{j+1} \frac{d}{d x}}\left(b_{0}^{(i)}\right)^{x} \frac{d}{d x} x\right|_{x=\frac{-1}{w}} & & \text { if } r_{i}=\infty
\end{aligned}
$$


We denote the set of all such $Q$ as $\mathcal{T}_{\mathbb{D}}\left(n_{-}, n_{+}\right)$. Let $\mathcal{T}_{\mathbb{D}}:=\left\{\mathcal{T}_{\mathbb{D}}\left(n_{-}, n_{+}\right)\right\}_{n_{-}, n_{+} \in \mathbb{N}}$. The automorphism group of $\mathbb{H}, S L(2, \mathbb{R})$, naturally acts on $\mathcal{T}_{\mathbb{D}}\left(n_{-}, n_{+}\right)$. It is clear that

$$
\mathbb{D}\left(n_{-}, n_{+}\right)=\mathcal{T}_{\mathbb{K}}\left(n_{-}, n_{+}\right) / S L(2, \mathbb{R}) .
$$

We denote the quotient map as $\pi_{\mathbb{D}}$. The identity $I_{\mathbb{D}} \in \mathbb{D}(1,1)$ is given by

$$
I_{\mathbb{D}}=\pi_{\mathbb{D}}((\infty, 1, \mathbf{0}) \mid(0,1, \mathbf{0})) .
$$

The composition $i \circ_{j}$ is provided by the sewing operation ${ }_{i} \infty_{-j}$ [HKo1]. In particular, for $n_{1}, m_{2} \geq 1, P \in \mathbb{D}\left(m_{-}, m_{+}\right)$and $Q \in \mathbb{D}\left(n_{-}, n_{+}\right), P_{i} \infty_{-j} Q$ is the disk with strips obtained by sewing the $i^{\text {th }}$ positively oriented puncture of $P$ with the $j^{\text {th }}$ negatively oriented puncture of $Q$. The $S_{n_{-}} \times S_{n_{+}}$-action on $\mathbb{D}\left(n_{-}, n_{+}\right)\left(\right.$or $\left.\mathcal{T}\left(n_{-}, n_{+}\right)\right)$is the natural one. The set

$$
\left\{((\infty, 1, \mathbf{0}) \mid(0, a, \mathbf{0})) \mid a \in \mathbb{R}_{+}\right\}
$$

together with multiplication $1 \infty_{1}$ is a group which can be canonically identified with group $\mathbb{R}_{+}$. It is clear that $\mathbb{D}$ is a $\mathbb{R}_{+}$-rescalable partial dioperad. We call it the disk partial dioperad.

$\mathbb{D}$ can be naturally embedded to $\mathbb{K}$ as a sub-dioperad. The $\mathbb{C}$-extension $\mathbb{D}^{c}$ of $\mathbb{D}$ for $c \in \mathbb{C}$ is just the restriction of the line bundle $\mathbb{K}^{c}$ on $\mathbb{D}$. $\mathbb{D}^{c}$ is also a $\mathbb{R}_{+}$-rescalable partial dioperad and a partial sub-dioperad of $\mathbb{K}^{c}$. We denote the canonical section on $\mathbb{D} \rightarrow \mathbb{D}^{c}$ as $\psi_{\mathbb{D}}$.

Now we discuss an example of a partial nonassociative dioperad which is important for us. Let $U=\oplus_{n \in J} U_{(n)}$ be a graded vector space and $J$ an index set. We denote the projection $U \rightarrow U_{(n)}$ as $P_{n}$. Now we consider a family of spaces of multi-linear maps $\mathbb{E}_{U}=\left\{\mathbb{E}_{U}(m, n)\right\}_{m, n \in \mathbb{N}}$, where

$$
\mathbb{E}_{U}(m, n):=\operatorname{Hom}_{\mathbb{C}}\left(U^{\otimes m}, \overline{U^{\otimes n}}\right) .
$$

For $f \in \mathbb{E}_{U}(m, n), g_{j} \in \mathbb{E}_{U}\left(k_{j}, l_{j}\right)$ and $u_{p_{j}}^{(j)} \in U, 1 \leq p_{j} \leq l_{j}, j=1, \ldots, n$, we say that

$$
\begin{aligned}
& \Gamma_{\left(i_{1}, \ldots, i_{n}\right)}\left(f ; g_{1}, \ldots, g_{n}\right)\left(u_{1}^{(1)} \otimes \cdots \otimes u_{l_{n}}^{(n)}\right) \\
& \quad:=\sum_{s_{1}, \ldots, s_{n} \in J} f\left(P_{s_{1}} g_{1}\left(u_{1}^{(1)} \otimes \cdots \otimes u_{l_{1}}^{(1)}\right) \otimes \cdots \otimes P_{s_{n}} g_{n}\left(u_{1}^{(n)} \otimes \cdots \otimes u_{l_{n}}^{(n)}\right)\right)
\end{aligned}
$$

is well-defined if the multiple sum converges absolutely. This gives rise to a partially defined substitution map, for $1 \leq i_{j} \leq k_{j}, j=1, \ldots, n$,

$$
\Gamma_{i_{1}, \ldots, i_{n}}: \mathbb{E}_{U}(m, n) \otimes \mathbb{E}_{U}\left(k_{1}, l_{1}\right) \otimes \cdots \otimes \mathbb{E}_{U}\left(k_{n}, l_{n}\right) \rightarrow \mathbb{E}_{U}(m+k-n, l),
$$

where $k=k_{1}+\cdots+k_{n}$ and $l=l_{1}+\cdots+l_{n}$. In general, the compositions of three substitution maps are not associative. The permutation groups actions on $\mathbb{E}_{U}$ are the usual one. Let $\Gamma=\left\{\Gamma_{i_{1}, \ldots, i_{n}}\right\}$. It is clear that $\left(\mathbb{E}_{U}, \Gamma, \mathrm{id}_{U}\right)$ is a partial nonassociative dioperad. We often denote it simply by $\mathbb{E}_{U}$.

Definition 1.8. Let $\left(\mathcal{P}, \gamma_{\mathcal{P}}, I_{\mathcal{P}}\right)$ be a partial dioperad. $A \mathcal{P}$-algebra $(U, v)$ consists of a graded vector space $U$ and a morphism of partial nonassociative dioperad $\nu: \mathcal{P} \rightarrow \mathbb{E}_{U}$. 
When $U=\oplus_{n \in J} U_{(n)}$ is a completely reducible module for a group $G, J$ is the set of equivalent classes of irreducible $G$-modules and $U_{(n)}$ is a direct sum of irreducible $G$-modules of equivalent class $n \in J$, we denote $\mathbb{E}_{U}$ by $\mathbb{E}_{U}^{G}$.

Definition 1.9. Let $\left(\mathcal{P}, \gamma_{\mathcal{P}}, I_{\mathcal{P}}\right)$ be a $G$-rescalable partial dioperad. A $G$-rescalable $\mathcal{P}$-algebra $(U, v)$ is a $\mathcal{P}$-algebra and the morphism $v: \mathcal{P} \rightarrow \mathbb{E}_{U}^{G}$ is so that $\left.v\right|_{G}$ : $G \rightarrow$ End $U$ coincides with the given $G$-module structure on $U$.

1.2. Conformal full field algebras. Let $\left(V^{L}, Y_{V^{L}}, \mathbf{1}^{L}, \omega^{L}\right)$ and $\left(V^{R}, Y_{V^{R}}, \mathbf{1}^{R}, \omega^{R}\right)$ be two vertex operator algebras with central charge $c^{L}$ and $c^{R}$ respectively, satisfying the conditions in Theorem 0.1. Let $\left(V_{c l}, m_{c l}, \iota_{c l}\right)$ be a conformal full field algebra over $V^{L} \otimes V^{R}$. A bilinear form $(\cdot, \cdot)_{c l}$ on $V_{c l}$ is invariant [Ko1] if, for any $u, w_{1}, w_{2} \in V_{c l}$,

$$
\begin{aligned}
& \left(w_{2}, \mathbb{Y}_{f}(u ; x, \bar{x}) w_{1}\right)_{c l} \\
& =\left(\mathbb{Y}_{f}\left(e^{-x L^{L}(1)} x^{-2 L^{L}(0)} \otimes e^{-\bar{x} L^{R}(1)} \bar{x}^{-2 L^{R}(0)} u ; e^{\pi i} x^{-1}, e^{-\pi i} \bar{x}^{-1}\right) w_{2}, w_{1}\right)_{c l},
\end{aligned}
$$

or equivalently,

$$
\begin{aligned}
& \left(\mathbb{Y}_{f}\left(u ; e^{\pi i} x, e^{-\pi i} \bar{x}\right) w_{2}, w_{1}\right)_{c l} \\
& \quad=\left(w_{2}, \mathbb{Y}_{f}\left(e^{x L^{L}(1)} x^{-2 L^{L}(0)} \otimes e^{\bar{x} L^{R}(1)} \bar{x}^{-2 L^{R}(0)} u ; x^{-1}, \bar{x}^{-1}\right) w_{1}\right)_{c l} .
\end{aligned}
$$

We showed in [Ko1] that an invariant bilinear form on $V_{c l}$ is automatically symmetric. Namely, for $u_{1}, u_{2} \in V_{c l}$, we have

$$
\left(u_{1}, u_{2}\right)_{c l}=\left(u_{2}, u_{1}\right)_{c l}
$$

$V_{c l}$ has a countable basis. We choose it to be $\left\{e_{i}\right\}_{i \in \mathbb{N}}$. Assume that $(\cdot, \cdot)_{c l}$ is also nondegenerate, we also have the dual basis $\left\{e^{i}\right\}_{i \in \mathbb{N}}$. Then we define a linear map $\Delta_{c l}$ : $\mathbb{C} \rightarrow \overline{V_{c l} \otimes V_{c l}}$ as follows:

$$
\Delta_{c l}: 1 \mapsto \sum_{i \in \mathbb{N}} e_{i} \otimes e^{i}
$$

The correlation functions maps $m_{c l}^{(n)}, n \in \mathbb{N}$ of $V_{c l}$ are canonically determined by $\mathbb{Y}$ and the identity $\mathbf{1}_{c l}:=\iota_{c l}\left(\mathbf{1}^{L} \otimes \mathbf{1}^{R}\right)$ [Ko2].

For $Q \in \mathcal{T}_{\mathbb{K}}\left(n_{-}, n_{+}\right)$given in (1.3), we define, for $\lambda \in \mathbb{C}$,

$$
v_{c l}\left(\lambda \psi_{\mathbb{K}}\left(\pi_{\mathbb{K}}(Q)\right)\right)\left(u_{1} \otimes \cdots \otimes u_{n_{+}}\right)
$$

in the following three cases: 
1. If $z_{k} \neq \infty$ for $k=-n_{-}, \ldots,-1,1, \ldots, n_{+},(1.21)$ is given by

$$
\begin{aligned}
& \lambda \sum_{i_{1}, \ldots, i_{n_{-}} \in \mathbb{N}}\left(\mathbf{1}_{c l}, m_{c l}^{\left(n_{-}+n_{+}\right)}\left(e^{\left.-L_{+}^{L}\left(A^{(-1)}\right)-L_{+}^{R} \overline{\left(A^{(-1)}\right.}\right)}\left(a_{0}^{(-1)}\right)^{-L^{L}(0) \overline{a_{0}^{(-1)}}-L^{R}(0)} e_{i_{1}},\right.\right.
\end{aligned}
$$



$$
\begin{aligned}
& e^{-L_{+}^{L}\left(A^{(1)}\right)-L_{+}^{R}\left(\overline{A^{(1)}}\right)}\left(a_{0}^{(1)}\right)^{-L^{L}(0)} \overline{a_{0}^{(1)}}-L^{R}(0) u_{1},
\end{aligned}
$$



$$
\begin{aligned}
& \left.\left.z_{-1}, \bar{z}_{-1}, \ldots, z_{-n_{-}}, \bar{z}_{-n_{-}}, z_{1}, \bar{z}_{1}, \ldots, z_{n_{+}}, \bar{z}_{n_{+}}\right)\right)_{c l} e^{i_{1}} \otimes \cdots \otimes e^{i_{n_{-}}},
\end{aligned}
$$

where $L_{+}^{L}(A)=\sum_{j=1}^{\infty} A_{j} L_{+}^{L}(j)$ and $L_{+}^{R}(A)=\sum_{j=1}^{\infty} A_{j} L_{+}^{R}(j)$ for $A=\left(A_{1}, A_{2}, \ldots\right) \in \prod_{j=1}^{\infty} \mathbb{C}$;

2. If $\exists k \in\left\{-n_{-}, \ldots,-1\right\}$ such that $z_{k}=\infty$ (recall (1.5)), (1.21) is given by the formula obtained from (1.22) by exchanging $\mathbf{1}_{c l}$ in (1.22) with

$$
e^{-L_{+}^{L}\left(A^{(k)}\right)-L_{+}^{R}\left(\overline{A^{(k)}}\right)}\left(a_{0}^{(k)}\right)^{-L^{L}(0)} \overline{a_{0}^{(k)}-L^{R}(0)} e_{i_{-k}} ;
$$

3. If $\exists k \in\left\{1, \ldots, n_{+}\right\}$such that $z_{k}=\infty$ (recall (1.5)), (1.21) is given by the formula obtained from (1.22) by exchanging $\mathbf{1}_{c l}$ in (1.22) with

$$
e^{-L_{+}^{L}\left(A^{(k)}\right)-L_{+}^{R} \overline{A^{(k)}}}\left(a_{0}^{(k)}\right)^{-L^{L}(0) \overline{a_{0}^{(k)}}-L^{R}(0)} u_{k} .
$$

The following result is proved in [Ko1].

Proposition 1.10. The map $v_{c l}$ is $S L(2, \mathbb{C})$-invariant.

Hence $v_{c l}$ induces a map $\tilde{\mathbb{K}}^{c^{L}} \otimes \overline{\tilde{\mathbb{K}}^{c^{R}}} \rightarrow \mathbb{E}_{V_{c l}}^{\mathbb{C}^{\times}}$, which is still denoted as $v_{c l}$. Some interesting special cases are listed below:

$$
\begin{aligned}
v_{c l}\left(\psi_{\mathbb{K}}(\hat{\mathbb{C}})\right) & =\left(\mathbf{1}_{c l}, \mathbf{1}_{c l}\right)_{c l} \mathrm{id}_{\mathbb{C}}, \\
v_{c l}\left(\psi_{\mathbb{K}}\left(\pi_{\mathbb{K}}\left(((\infty, 1, \mathbf{0}) \mid)_{\mathbb{K}}\right)\right)\right. & =\mathbf{1}_{c l}, \\
v_{c l}\left(\psi_{\mathbb{K}}\left(\pi_{\mathbb{K}}\left((\mid(\infty, 1, \mathbf{0}),(0,1, \mathbf{0}))_{\mathbb{K}}\right)\right)(u \otimes v)\right. & =(u, v)_{c l}, \\
v_{c l}\left(\psi_{\mathbb{K}}\left(\pi_{\mathbb{K}}\left(((\infty, 1, \mathbf{0}),(0,1, \mathbf{0}) \mid)_{\mathbb{K}}\right)\right)\right) & =\Delta_{c l}, \\
v_{c l}\left(\psi_{\mathbb{K}}\left(\pi_{\mathbb{K}}\left(((\infty ; 1, \mathbf{0}) \mid(z ; 1, \mathbf{0}),(0 ; 1, \mathbf{0}))_{\mathbb{K}}\right)\right)\right)(u \otimes v) & =\mathbb{Y}(u ; z, \bar{z}) v,
\end{aligned}
$$

where $\hat{\mathbb{C}}$ is the single element in $\mathbb{K}(0,0)$.

Definition 1.11. A $\tilde{\mathbb{K}}^{c^{L}} \otimes \overline{\tilde{\mathbb{K}}^{\overline{c^{R}}}}$-algebra $(U, v)$ is called smooth if

1. $U=\oplus_{m, n \in \mathbb{R}} U_{(m, n)}$ is a completely reducible $\mathbb{C}^{\times}$-module, where $z \cdot u=z^{m} \bar{z}^{n} u$, $\forall z \in \mathbb{C}^{\times}, u \in U_{(m, n)}$.

2. $\operatorname{dim} U_{(m, n)}<\infty, \forall m, n \in \mathbb{R}$ and $\operatorname{dim} U_{(m, n)}=0$ for $m$ or $n$ sufficiently small.

3. $v$ is linear on fiber and smooth on the base space $\mathbb{K}$.

Theorem 2.7 in [Ko1] can be restated as the following theorem.

Theorem 1.12. $\left(V_{c l}, v_{c l}\right)$ is a smooth $\tilde{\mathbb{K}}^{c^{L}} \otimes \overline{\tilde{\mathbb{K}}^{\overline{c^{R}}}}$-algebra. 
1.3. Open-string vertex operator algebras. Let $\left(V_{o p}, Y_{o p}, \mathbf{1}_{o p}, \omega_{o p}\right)$ be an open-string vertex operator algebra. For $r>0$ and $v_{1}, v_{2} \in V_{o p}$, we define $Y_{o p}\left(v_{1},-r\right) v_{2}$ by

$$
Y_{o p}\left(v_{1},-r\right) v_{2}:=e^{-r L(-1)} Y_{o p}\left(v_{2}, r\right) v_{1} \text {. }
$$

Remark 1.13. Taking the analogy between the open-string vertex operator algebra and associative algebra, $Y_{o p}(\cdot,-r) \cdot$ corresponds to the opposite product [HKo1].

An invariant bilinear form on an open-string vertex operator algebra $V_{o p}$ is a bilinear form $(\cdot, \cdot)_{o p}$ on $V_{o p}$ satisfying the following properties:

$$
\begin{aligned}
& \left(v_{3}, Y_{o p}\left(v_{1}, r\right) v_{2}\right)_{o p}=\left(Y_{o p}\left(e^{-r L(1)} r^{-2 L(0)} v_{1},-r^{-1}\right) v_{3}, v_{2}\right)_{o p}, \\
& \left(Y_{o p}\left(v_{1}, r\right) v_{3}, v_{2}\right)_{o p}=\left(v_{3}, Y_{o p}\left(e^{-r L(1)} r^{-2 L(0)} v_{1},-r^{-1}\right) v_{2}\right)_{o p}
\end{aligned}
$$

for $r>0$ and $v_{1}, v_{2}, v_{3} \in V_{o p}$.

\section{Lemma 1.14.}

$$
\left(v_{1}, v_{2}\right)_{o p}=\left(v_{2}, v_{1}\right)_{o p}
$$

Proof. The proof is exactly the same as that of Proposition 2.3 in [Ko1].

We further assume that $(\cdot, \cdot)_{o p}$ is nondegenerate. Let $\left\{f_{i}\right\}_{i \in \mathbb{R}}$ be a basis of $V_{o p}$ and $\left\{f^{i}\right\}_{i \in \mathbb{R}}$ its dual basis. We define linear map $\Delta_{o p}: \mathbb{C} \rightarrow \overline{V_{c l} \otimes V_{c l}}$ as follows:

$$
\Delta_{o p}: 1 \mapsto \sum_{i \in \mathbb{R}} f_{i} \otimes f^{i}
$$

The open-string vertex operator algebra $\left(V_{o p}, Y_{o p}, \mathbf{1}_{o p}, \omega_{o p}\right)$ naturally gives a boundary field algebra $\left(V_{o p}, m_{o p}, \mathbf{d}_{o p}, D_{o p}\right)$ in which the correlation-function maps $m_{o p}^{(n)}$, $n \in \mathbb{N}$ are completely determined by $Y_{o p}$ and $\mathbf{1}_{o p}[\mathrm{Ko} 2]$.

For any $Q \in \mathcal{T}_{\mathbb{D}}\left(n_{-}, n_{+}\right)$given in (1.10). Let $\alpha$ be a bijective map

$$
\left\{-n_{-}, \ldots,-1,1, \ldots, n_{+}\right\} \stackrel{\alpha}{\rightarrow}\left\{1, \ldots, n_{-}+n_{+}\right\}
$$

so that $s_{1}, \ldots, s_{n_{-}+n_{+}}$, defined by $s_{i}:=r_{\alpha^{-1}(i)}$, satisfy $\infty \geq s_{1}>\cdots>s_{n_{-}+n_{+}} \geq 0$. Then we define, for $\lambda \in \mathbb{C}$,

$$
v_{o p}\left(\lambda \psi_{\mathbb{D}}\left(\pi_{\mathbb{D}}(Q)\right)\right)\left(v_{1} \otimes \cdots \otimes v_{n_{+}}\right)
$$

as follows:

1. If $r_{k} \neq \infty, \forall k=-n_{-}, \ldots,-1,1, \ldots, n_{+},(1.31)$ is given by

$$
\lambda \sum_{i_{1}, \ldots, i_{n_{-}} \in \mathbb{R}}\left(\mathbf{1}_{o p}, m_{o p}^{\left(n_{-}+n_{+}\right)}\left(w_{1}, \ldots, w_{n_{-}+n_{+}} ; s_{1}, \ldots, s_{n_{-}+n_{+}}\right)\right)_{o p} f^{i_{1}} \otimes \cdots \otimes f^{i_{n_{-}}},
$$

where $w_{\alpha(p)}=e^{-L_{+}\left(B^{(p)}\right)}\left(b_{0}^{(p)}\right)^{-L(0)} f_{i_{-p}}$ and $w_{\alpha(q)}=e^{-L_{+}\left(B^{(q)}\right)}\left(b_{0}^{(q)}\right)^{-L(0)} v_{q}$ for $p=-1, \cdots-n_{-}$and $q=1, \ldots, n_{+}$;

2. If $\exists k \in\left\{-n_{-}, \ldots,-1,1, \ldots, n_{+}\right\}$such that $r_{k}=\infty,(1.31)$ is given by the formula obtained from (1.32) by exchanging the $\mathbf{1}_{o p}$ with $w_{\alpha(k)}$. 
Proposition 1.15. $v_{\text {op }}$ is $S L(2, \mathbb{R})$ invariant.

Proof. The proof is the same as that of Proposition 1.10.

Hence $v_{o p}$ induces a map $\tilde{\mathbb{D}}^{c} \rightarrow \mathbb{E}_{V_{o p}}^{\mathbb{R}_{+}}$, which is still denoted as $v_{o p}$. Some interesting special cases are listed explicitly below:

$$
\begin{aligned}
v_{o p}\left(\psi_{\mathbb{D}}(\hat{\mathbb{H}})\right) & =\left(\mathbf{1}_{o p}, \mathbf{1}_{o p}\right)_{o p} \mathrm{id}_{\mathbb{C}}, \\
v_{o p}\left(\psi_{\mathbb{D}}\left(\pi_{\mathbb{D}}(((\infty, 1, \mathbf{0}) \mid))\right)\right) & =\mathbf{1}_{o p}, \\
v_{o p}\left(\psi_{\mathbb{D}}\left(\pi_{\mathbb{D}}((\mid(\infty, 1, \mathbf{0}),(0,1, \mathbf{0})))\right)\right)(u \otimes v) & =(u, v)_{o p}, \\
v_{o p}\left(\psi_{\mathbb{D}}\left(\pi_{\mathbb{D}}(((\infty, 1, \mathbf{0}),(0,1, \mathbf{0}) \mid))\right)\right) & =\Delta_{o p}, \\
v_{o p}\left(\psi_{\mathbb{D}}\left(\pi_{\mathbb{D}}(((\infty ; 1, \mathbf{0}) \mid(r ; 1, \mathbf{0}),(0 ; 1, \mathbf{0})))\right)\right)(u \otimes v) & =Y_{o p}(u, r) v,
\end{aligned}
$$

where $\hat{\mathbb{H}}$ is the single element in $\mathbb{D}(0,0)$ and $r>0$.

Definition 1.16. $A \tilde{\mathbb{D}}^{c}$-algebra $(U, v)$ is called smooth if

1. $U=\oplus_{n \in \mathbb{R}} U_{(n)}$ is a completely reducible $\mathbb{R}_{+}$-module, where $r \cdot u=r^{n} u$, $\forall r \in \mathbb{R}_{+}, u \in U_{(m, n)}$.

2. $\operatorname{dim} U_{(n)}<\infty, \forall n \in \mathbb{R}$ and $\operatorname{dim} U_{(n)}=0$ for $n$ sufficiently small.

3. $v$ is linear on fiber and smooth on the base space $\mathbb{D}$.

Theorem 1.17. $\left(V_{o p}, v_{o p}\right)$ is a smooth $\tilde{\mathbb{D}}^{c}$-algebra.

Proof. The proof is same as that of Theorem 1.12.

\section{Swiss-Cheese Partial Dioperad}

In Sect. 3.1, we introduce the notion of a 2-colored partial dioperad and algebra over it. In Sect. 3.2, we study a special example of a 2-colored partial dioperad called a Swiss-cheese partial dioperad $\mathbb{S}$ and its $\mathbb{C}$-extension $\tilde{\mathbb{S}}^{c}$. In Sect. 3.3, we show that an open-closed field algebra over $V$ equipped with nondegenerate invariant bilinear forms canonically gives an algebra over $\tilde{\mathbb{S}}^{c}$. In Sect. 3.4, we define boundary states in such algebra and show that some of the boundary states are Ishibashi states.

\subsection{2-colored (partial) dioperads.}

Definition 2.1. A right module over a dioperad $\left(\mathcal{Q}, \gamma_{\mathcal{Q}}, I_{\mathcal{Q}}\right)$, or a right $\mathcal{Q}$-module, is a family of sets $\{\mathcal{P}(m, n)\}_{m, n \in \mathbb{N}}$ with an $S_{m} \times S_{n}$-action on each set $\mathcal{P}(m, n)$ and substitution maps:

$$
\begin{aligned}
\mathcal{P}(m, n) \times \mathcal{Q}\left(k_{1}, l_{1}\right) \times \cdots \times \mathcal{Q}\left(k_{n}, l_{n}\right) \stackrel{\gamma_{\left(i_{1}, \ldots, i_{n}\right)}}{\longrightarrow} \mathcal{P}\left(m+k_{1} \cdots+k_{n}-n, l_{1}+\cdots+l_{n}\right), \\
\left(P, Q_{1}, \ldots, Q_{n}\right) \quad \mapsto \quad \gamma_{\left(i_{1}, \ldots, i_{n}\right)}\left(P ; Q_{1}, \ldots, Q_{n}\right)
\end{aligned}
$$

for $m, n, l_{1}, \ldots, l_{n} \in \mathbb{N}, k_{1}, \ldots, k_{n} \in \mathbb{Z}_{+}$and $1 \leq i_{j} \leq k_{j}, j=1, \ldots, n$, satisfying the right unit property, the associativity and the permutation axioms of dioperad but with the right action of $\mathcal{P}$ on itself in the definition of dioperad replaced by that of $\mathcal{Q}$. 
Homomorphism and isomorphism between two right $\mathcal{Q}$-modules can be naturally defined. The right module over a partial dioperad can also be defined in the usual way.

Definition 2.2. Let $\mathcal{Q}$ be a $G$-rescalable partial dioperad. A right $\mathcal{Q}$-module is called $G$-rescalable if for any $P \in \mathcal{P}\left(m_{-}, m_{+}\right), Q_{i} \in \mathcal{Q}\left(n_{-}^{(i)}, n_{+}^{(i)}\right)$ and $1 \leq j_{i} \leq n_{-}^{(i)}$, $i=1, \ldots, m_{+}$, there exist $g_{j} \in G, j=1, \ldots, m_{+}$such that

$$
\gamma_{\left(j_{1}, \ldots, j_{m_{+}}\right)}\left(P ; \gamma_{\left(j_{1}\right)}\left(g_{1} ; Q_{1}\right), \ldots, \gamma_{\left(j_{m_{+}}\right)}\left(g_{m_{+}} ; Q_{m_{+}}\right)\right)
$$

is well-defined.

Definition 2.3. A 2-colored dioperad consists of a dioperad $\left(\mathcal{Q}, \gamma_{\mathcal{Q}}, I_{\mathcal{Q}}\right)$ and a family of sets $\mathcal{P}\left(n_{-}^{B}, n_{+}^{B} \mid n_{-}^{I}, n_{+}^{I}\right)$ equipped with a $S_{n_{-}^{B}} \times S_{n_{+}^{B}} \times S_{n_{-}^{I}} \times S_{n_{+}^{I}}$-action for $n_{ \pm}^{B}, n_{ \pm}^{I} \in \mathbb{N}$, a distinguished element $I_{\mathcal{P}} \in \mathcal{P}(1,1 \mid 0,0)$, and maps

$$
\begin{aligned}
& \mathcal{P}\left(m_{-}, m_{+} \mid n_{-}, n_{+}\right) \times \mathcal{P}\left(k_{-}^{(1)}, k_{+}^{(1)} \mid l_{-}^{(1)}, l_{+}^{(1)}\right) \times \cdots \times \mathcal{P}\left(k_{-}^{\left(m_{+}\right)}, k_{+}^{\left(m_{+}\right)} \mid l_{-}^{\left(m_{+}\right)}, l_{+}^{\left(m_{+}\right)}\right) \stackrel{\gamma_{\left(i_{1}, \ldots, i_{\left.m_{+}\right)}\right.}^{\longrightarrow}}{\longrightarrow} \mathcal{P}\left(m_{-}-m_{+}+k_{-}, k_{+} \mid n_{-}+l_{-}, n_{+}+l_{+}\right), \\
& \mathcal{P}\left(m_{-}, m_{+} \mid n_{-}, n_{+}\right) \times \mathcal{Q}\left(p_{-}^{(1)}, p_{+}^{(1)}\right) \times \cdots \times \mathcal{Q}\left(p_{-}^{\left(n_{+}\right)}, p_{+}^{(n)}\right) \\
& \stackrel{\gamma_{\left(j_{1}, \ldots, j_{\left.n_{+}\right)}\right)}^{\longrightarrow}}{\longrightarrow} \mathcal{P}\left(m_{-}, m_{+} \mid n_{-}-n_{+}+p_{-}, p_{+}\right),
\end{aligned}
$$

where $k_{ \pm}=k_{ \pm}^{(1)}+\cdots+k_{ \pm}^{\left(m_{+}\right)}, l_{ \pm}=l_{ \pm}^{(1)}+\cdots+l_{ \pm}^{\left(m_{+}\right)}$and $p_{ \pm}=p_{ \pm}^{(1)}+\cdots+p_{ \pm}^{\left(n_{+}\right)}$, for $1 \leq i_{r} \leq k_{-}^{(r)}, r=1, \ldots, m_{+}, 1 \leq j_{s} \leq p_{-}^{(s)}, s=1, \ldots, n_{+}$, satisfying the following axioms:

1. The family of sets $\mathcal{P}:=\left\{\mathcal{P}\left(m_{-}, m_{+}\right)\right\}_{m_{-}, m_{+} \in \mathbb{N}}$, where

$$
\mathcal{P}\left(m_{-}, m_{+}\right):=\cup_{n_{-}, n_{+} \in \mathbb{N}} \mathcal{P}\left(m_{-}, m_{+} \mid n_{-}, n_{+}\right),
$$

equipped with the natural $S_{m_{-}} \times S_{m_{+}}$-action on $\mathcal{P}\left(m_{-}, m_{+}\right)$, together with identity element $I_{\mathcal{P}}$ and the family of maps $\gamma^{B}:=\left\{\gamma_{\left(i_{1}, \ldots, i_{m_{+}}\right)}^{B}\right\}$ gives an dioperad.

2. The family of maps $\gamma^{I}:=\left\{\gamma_{\left(j_{1}, \ldots, j_{n_{+}}\right)}^{I}\right\}$ gives each $\mathcal{P}\left(m_{-}, m_{+}\right)$a right $\mathcal{Q}$-module structure for $m_{-}, m_{+} \in \mathbb{N}$.

We denote such a 2-colored dioperad as $\left(\mathcal{P} \mid \mathcal{Q},\left(\gamma^{B}, \gamma^{I}\right)\right)$. The 2-colored partial dioperad can be naturally defined. If the associativities of $\gamma_{\mathcal{Q}}, \gamma^{B}, \gamma^{I}$ do not hold, then it is called a 2-colored (partial) nonassociative dioperad.

Remark 2.4. If we restrict to $\{\mathcal{P}(1, m \mid 0, n)\}_{m, n \in \mathbb{N}}$ and $\{\mathcal{Q}(1, n)\}_{n \in \mathbb{N}}$, they simply gives a structure of 2-colored (partial) operad [V,Kont,Ko2].

Now we discuss an important example of the 2-colored partial nonassociative dioperad. Let $J_{1}, J_{2}$ be two index sets, $U_{i}=\oplus_{n \in J_{i}}\left(U_{i}\right)_{(n)}, i=1,2$ be two graded vector spaces. Consider two families of sets,

$$
\begin{aligned}
\mathbb{E}_{U_{2}}(m, n) & =\operatorname{Hom}_{\mathbb{C}}\left(U_{2}^{\otimes m}, \overline{U_{2}^{\otimes n}}\right) \\
\mathbb{E}_{U_{1} \mid U_{2}}\left(m_{-}, m_{+} \mid n_{-}, n_{+}\right) & =\operatorname{Hom}_{\mathbb{C}}\left(U_{1}^{\otimes m_{+}} \otimes U_{2}^{\otimes n_{+}}, \overline{U_{1}^{\otimes m_{-}} \otimes U_{2}^{\otimes n_{-}}}\right),
\end{aligned}
$$


for $m, n, m_{ \pm}, n_{ \pm} \in \mathbb{N}$. We denote both of the projection operators $U_{1} \rightarrow\left(U_{1}\right)_{(n)}$, $U_{2} \rightarrow\left(U_{2}\right)_{(n)}$ as $P_{n}$ for $n \in J_{1}$ or $J_{2}$. For $f \in \mathbb{E}_{U_{1} \mid U_{2}}\left(k_{-}, k_{+} \mid l_{-}, l_{+}\right), g_{i} \in \mathbb{E}_{U_{1} \mid U_{2}}\left(m_{-}^{(i)}\right.$, $\left.m_{+}^{(i)} \mid n_{-}^{(i)}, n_{+}^{(i)}\right), i=1, \ldots, k_{+}$, and $h_{j} \in \mathbb{E}_{U_{2}}\left(p_{-}^{(j)}, p_{+}^{(j)}\right), j=1, \ldots, l_{+}$, we say that $\Gamma_{\left(i_{1}, \ldots, i_{k_{+}}\right)}^{B}\left(f ; g_{1}, \ldots, g_{k_{+}}\right)\left(u_{1}^{(1)} \otimes \cdots \otimes v_{n_{+}^{\left(k_{+}\right)}}^{\left(k_{+}\right)} \otimes v_{1} \otimes \cdots \otimes v_{l_{+}}\right)$

$$
\begin{gathered}
:=\sum_{s_{1}, \ldots, s_{k} \in J_{1}} f\left(P_{s_{1}} g_{1}\left(u_{1}^{(1)} \otimes \cdots \otimes u_{m_{+}^{(1)}}^{(1)} \otimes v_{1}^{(1)} \otimes \cdots \otimes v_{n_{+}}^{(1)}\right) \otimes\right. \\
\cdots \otimes P_{s_{k_{+}}} g_{k_{+}}\left(u_{1}^{\left(k_{+}\right)} \otimes \cdots \otimes u_{m_{+}^{\left(k_{+}\right)}}^{\left(k_{+}\right)} \otimes v_{1}^{\left(k_{+}\right)} \otimes \cdots \otimes v_{n_{+}^{\left(k_{+}\right)}}^{\left(k_{k^{\prime}}\right)} \otimes v_{1} \otimes \cdots \otimes v_{l_{+}}\right) \\
\Gamma_{\left(j_{1}, \ldots, j_{l_{+}}\right)}^{I}\left(f ; h_{1}, \ldots, h_{l_{+}}\right)\left(u_{1} \otimes \cdots \otimes u_{k_{+}} \otimes w_{1}^{(1)} \otimes \cdots \otimes w_{p_{+}^{\left(l_{+}\right)}}^{\left(l_{+}\right)}\right)
\end{gathered}
$$

$$
\begin{array}{r}
:=\sum_{t_{1}, \ldots, t_{l} \in J_{2}} f\left(u_{1} \otimes \cdots \otimes u_{k_{+}} \otimes P_{t_{1}} h_{1}\left(w_{1}^{(1)} \otimes \cdots \otimes w_{p_{+}^{(1)}}^{(1)}\right)\right. \\
\left.\otimes \cdots \otimes P_{t_{l_{+}}} h_{l_{+}}\left(w_{1}^{\left(l_{+}\right)} \otimes \cdots \otimes w_{p_{+}^{\left(l_{+}\right)}}^{\left(l_{+}\right)}\right)\right)
\end{array}
$$

for $u_{j}^{(i)} \in U_{1}, v_{j}^{(i)}, w_{j}^{(i)} \in U_{2}$, are well-defined if each multiple sum is absolutely convergent. These give rise to partially defined substitution maps:

$$
\begin{aligned}
\mathbb{E}_{U_{1} \mid U_{2}}\left(k_{-},\right. & \left.k_{+} \mid l_{-}, l_{+}\right) \otimes \mathbb{E}_{U_{1} \mid U_{2}}\left(m_{-}^{(1)}, m_{+}^{(1)} \mid n_{-}^{(1)}, n_{+}^{(1)}\right) \otimes \ldots \otimes \mathbb{E}_{U_{1} \mid U_{2}}\left(m_{-}^{\left(k_{+}\right)}, m_{+}^{\left(k_{+}\right)} \mid n_{-}^{\left(k_{+}\right)}, n_{+}^{\left(k_{+}\right)}\right) \\
& \stackrel{\Gamma_{\left(i_{1}, \ldots, i_{k_{+}}\right)}^{B}}{\longrightarrow} \mathbb{E}_{U_{1} \mid U_{2}}\left(k_{-}-k_{+}+m_{-}, m_{+} \mid l_{-}+n_{-}, n_{+}\right), \\
\mathbb{E}_{U_{1} \mid U_{2}}\left(k_{-},\right. & \left.k_{+} \mid l_{-}, l_{+}\right) \otimes \mathbb{E}_{U_{2}}\left(p_{-}^{(1)}, p_{+}^{(1)}\right) \otimes \cdots \otimes \mathbb{E}_{U_{2}}\left(p_{-}^{\left(l_{+}\right)}, p_{+}^{\left(l_{-}\right)}\right) \\
& \stackrel{\Gamma_{\left(j_{1}+\cdots+j_{l_{+}}\right)}^{I}}{\longrightarrow} \mathbb{E}_{U_{1} \mid U_{2}}\left(k_{-}, k_{+} \mid l_{-}-l_{+}+p_{-}^{(1)}+\cdots+p_{-}^{\left(l_{+}\right)}, p_{+}^{(1)}+\cdots+p_{+}^{\left(l_{+}\right)}\right),
\end{aligned}
$$

where $m_{ \pm}=m_{ \pm}^{(1)}+\cdots+m_{ \pm}^{\left(k_{+}\right)}$and $n_{ \pm}=n_{ \pm}^{(1)}+\cdots+n_{ \pm}^{\left(k_{+}\right)}$. In general, $\Gamma_{\left(i_{1}, \ldots, i_{k_{+}}\right)}^{B}$ and $\Gamma_{\left(j_{1}+\cdots+j_{l_{+}}\right)}^{I}$ do not satisfy the associativity. Let

$$
\mathbb{E}_{U_{1} \mid U_{2}}=\left\{\mathbb{E}_{U_{1} \mid U_{2}}\left(m_{-}, m_{+} \mid n_{-}, n_{+}\right)\right\}_{m_{ \pm}, n_{ \pm} \in \mathbb{N}},
$$

$\mathbb{E}_{U_{2}}=\left\{\mathbb{E}_{U_{2}}(n)\right\}_{n \in \mathbb{N}}, \Gamma^{B}:=\left\{\Gamma_{\left(i_{1}, \ldots, i_{n}\right)}^{B}\right\}$ and $\Gamma^{I}:=\left\{\Gamma_{\left(j_{1}, \ldots, j_{n}\right)}^{I}\right\}$. It is obvious that $\left(\mathbb{E}_{U_{1} \mid U_{2}} \mid \mathbb{E}_{U_{2}},\left(\Gamma^{B}, \Gamma^{I}\right)\right)$ is a 2-colored partial nonassociative operad.

Let $U_{1}$ be a completely reducible $G_{1}$-module and $U_{2}$ a, completely reducible $G_{2}$-module. Namely, $U_{1}=\oplus_{n_{1} \in J_{1}}\left(U_{1}\right)_{\left(n_{1}\right)}, U_{2}=\oplus_{n_{2} \in J_{2}}\left(U_{2}\right)_{\left(n_{2}\right)}$ where $J_{i}$ is the set of equivalent classes of irreducible $G_{i}$-modules and $\left(U_{i}\right)_{\left(n_{i}\right)}$ is a direct sum of irreducible $G_{i}$-modules of equivalent class $n_{i}$ for $i=1,2$. In this case, we denote $\mathbb{E}_{U_{1} \mid U_{2}}$ by $\mathbb{E}_{U_{1} \mid U_{2}}^{G_{1} \mid G_{2}}$.

Definition 2.5. A homomorphism between two 2-colored (partial, nonassociative) dioperads

$$
\left(\mathcal{P}_{i} \mid \mathcal{Q}_{i},\left(\gamma_{i}^{B}, \gamma_{i}^{I}\right)\right), i=1,2
$$

consists of two (partial, nonassociative) dioperad homomorphisms:

$$
v_{\mathcal{P}_{1} \mid \mathcal{Q}_{1}}: \mathcal{P}_{1} \rightarrow \mathcal{P}_{2}, \quad \text { and } \quad v_{\mathcal{Q}_{1}}: \mathcal{Q}_{1} \rightarrow \mathcal{Q}_{2}
$$

such that $\nu_{\mathcal{P}_{1} \mid \mathcal{Q}_{1}}: \mathcal{P}_{1} \rightarrow \mathcal{P}_{2}$, where $\mathcal{P}_{2}$ has a right $\mathcal{Q}_{1}$-module structure induced by a dioperad homomorphism $v_{\mathcal{Q}_{1}}$, is also a right $\mathcal{Q}_{1}$-module homomorphism. 
Definition 2.6. An algebra over a 2-colored partial dioperad $\left(\mathcal{P} \mid \mathcal{Q},\left(\gamma^{B}, \gamma^{I}\right)\right)$, or a $\mathcal{P} \mid \mathcal{Q}$-algebra consists of two graded vector spaces $U_{1}, U_{2}$ and a 2 -colored partial nonassociative dioperad homomorphism $\left(\nu_{\mathcal{P} \mid \mathcal{Q}}, v_{\mathcal{Q}}\right):\left(\mathcal{P} \mid \mathcal{Q},\left(\gamma^{B}, \gamma^{I}\right)\right) \rightarrow\left(\mathbb{E}_{U_{1} \mid U_{2}} \mid \mathbb{E}_{U_{2}}\right.$, $\left.\left(\Gamma^{B}, \Gamma^{I}\right)\right)$. We denote this algebra as $\left(U_{1} \mid U_{2}, \nu_{\mathcal{P} \mid \mathcal{Q}}, v_{\mathcal{Q}}\right)$.

Definition 2.7. If a 2-colored partial dioperad $(\mathcal{P} \mid \mathcal{Q}, \gamma)$ is so that $\mathcal{P}$ is a $G_{1}$-rescalable partial operad and a $G_{2}$-rescalable right $\mathcal{Q}$-module, then it is called $G_{1} \mid G_{2}$-rescalable.

Definition 2.8. $A G_{1} \mid G_{2}$-rescalable $\mathcal{P} \mid \mathcal{Q}$-algebra $\left(U_{1} \mid U_{2}, v_{\mathcal{P} \mid \mathcal{Q}}, v_{\mathcal{Q}}\right)$ is a $\mathcal{P} \mid \mathcal{Q}$-algebra so that $v_{\mathcal{P} \mid \mathcal{Q}}: \mathcal{P} \rightarrow \mathbb{E}_{U_{1} \mid U_{2}}^{G_{1} \mid G_{2}}$ and $v_{\mathcal{Q}}: \mathcal{Q} \rightarrow \mathbb{E}_{U_{2}}^{G_{2}}$ are partial nonassociative dioperad homomorphisms such that $\nu_{\mathcal{P} \mid \mathcal{Q}}: G_{1} \rightarrow$ End $U_{1}$ coincides with the $G_{1}$-module structure on $U_{1}$ and $v_{\mathcal{Q}}: G_{2} \rightarrow$ End $U_{2}$ coincides with the $G_{2}$-module structure on $U_{2}$.

2.2. Swiss-cheese partial dioperads. A disk with strips and tubes of type $\left(m_{-}, m_{+}\right.$; $\left.n_{-}, n_{+}\right)$[Ko2] is a disk consisting of $m_{+}\left(m_{-}\right)$ordered positively (negatively) oriented punctures on the boundary of the disk, and $n_{+}\left(n_{-}\right)$ordered positively (negatively) oriented punctures in the interior of the disk, and local coordinate map around each puncture. Two disks are conformal equivalent if there exists a biholomorphic map between them preserving order, orientation and local coordinates. We denote the moduli space of disks with strips and tubes of type $\left(m_{-}, m_{+} ; n_{-}, n_{+}\right)$as $\mathbb{S}\left(m_{-}, m_{+} \mid n_{-}, n_{+}\right)$.

We use the following notation:

$$
\begin{aligned}
& {\left[\left(\left(r_{-m_{-}}, b_{0}^{-m_{-}}, B^{\left(-m_{-}\right)}\right), \ldots,\left(r_{-1}, b_{0}^{-1}, B^{(-1)}\right) \mid\right.\right.} \\
& \left.\left(r_{1}, b_{0}^{1}, B^{(1)}\right), \ldots,\left(r_{m_{+}}, b_{0}^{m_{+}}, B^{\left(m_{+}\right)}\right)\right) \| \\
& \left(\left(z_{-n_{-}}, a_{0}^{-n_{-}}, A^{\left(-n_{-}\right)}\right), \ldots,\left(z_{-1}, a_{0}^{-1}, A^{(-1)}\right) \mid\right. \\
& \left.\left.\left(z_{1}, a_{0}^{1}, A^{(1)}\right), \ldots,\left(z_{n_{+}}, a_{0}^{n_{+}}, A^{\left(n_{+}\right)}\right)\right)\right]_{\mathbb{S}},
\end{aligned}
$$

where $r_{i} \in \hat{\mathbb{R}}, b_{0}^{(i)} \in \mathbb{R}^{\times}, B_{k}^{(i)} \in \mathbb{R}$ and $z_{j} \in \mathbb{H}, a_{0}^{(j)} \in \mathbb{C}^{\times}, A_{l}^{(j)} \in \mathbb{C}$ for all $i=-m_{-}, \ldots,-1,1, \ldots, m_{+}, j=-n_{-}, \ldots,-1,1, \ldots, n_{+}$and $k, l \in \mathbb{Z}_{+}$, to represent a disk with strips at $r_{i}$ with local coordinate map $f_{i}$ and tubes at $z_{j}$ with local coordinate map $g_{j}$ given as follows:

$$
\begin{array}{rlr}
f_{i}(w) & =\left.e^{\sum_{k} B_{k}^{(i)} x^{k+1} \frac{d}{d x}}\left(b_{0}^{(i)}\right)^{x} \frac{d}{d x} x\right|_{x=w-r_{i}} & \text { if } r_{i} \in \mathbb{R}, \\
& =\left.e^{\sum_{k} B_{k}^{(i)} x^{k+1} \frac{d}{d x}}\left(b_{0}^{(i)}\right)^{x \frac{d}{d x}} x\right|_{x=\frac{-1}{w}} \quad \text { if } r_{i}=\infty, \\
g_{j}(w) & =\left.e^{\sum_{k} A_{k}^{(j)} x^{k+1} \frac{d}{d x}}\left(a_{0}^{(j)}\right)^{x \frac{d}{d x}} x\right|_{x=w-z_{j}} .
\end{array}
$$

We denote the set of all such disks given in (2.4) as $\mathcal{T}_{\mathbb{S}}\left(m_{-}, m_{+} \mid n_{-}, n_{+}\right)$. The automorphisms of the upper half plane, which is $S L(2, \mathbb{R})$, change the disk (2.4) to a different but conformal equivalent disk. It is clear that we have

$$
\mathbb{S}\left(m_{-}, m_{+} \mid n_{-}, n_{+}\right)=\mathcal{T}_{\mathbb{S}}\left(m_{-}, m_{+} \mid n_{-}, n_{+}\right) / S L(2, \mathbb{R}) .
$$

Let $\mathbb{S}=\left\{\mathbb{S}\left(m_{-}, m_{+} \mid n_{-}, n_{+}\right)\right\}_{m_{-}, m_{+}, n_{-}, n_{+} \in \mathbb{N}}$. The permutation groups

$$
\left(S_{m_{-}} \times S_{m_{+}}\right) \times\left(S_{n_{-}} \times S_{n_{+}}\right)
$$


act naturally on $\mathbb{S}\left(m_{-}, m_{+} \mid n_{-}, n_{+}\right)$. There are so-called boundary sewing operations [HKo1,Ko2] on $\mathbb{S}$, denoted as $i \infty_{-j}^{B}$, which sews the $i^{\text {th }}$ positively oriented boundary puncture of the first disk with the $j^{\text {th }}$ negatively oriented boundary puncture of the second disk. Boundary sewing operations naturally induce the following maps:

$$
\begin{gathered}
\mathbb{S}\left(m_{-}, m_{+} \mid n_{-}, n_{+}\right) \times \mathbb{S}\left(k_{-}^{(1)}, k_{+}^{(1)} \mid l_{-}^{(1)}, l_{+}^{(1)}\right) \times \cdots \times \mathbb{S}\left(k_{-}^{\left(m_{+}\right)}, k_{+}^{\left(m_{+}\right)} \mid l_{-}^{\left(m_{+}\right)}, l_{+}^{\left(m_{+}\right)}\right) \\
\stackrel{\gamma_{\left(i_{1}, \ldots, i_{m_{+}}\right)}^{\longrightarrow}}{\longrightarrow} \mathbb{S}\left(m_{-}-m_{+}+k_{-}, k_{+} \mid n_{-}+l_{-}, n_{+}+l_{+}\right),
\end{gathered}
$$

where $k_{ \pm}=k_{ \pm}^{(1)}+\cdots+k_{ \pm}^{\left(m_{+}\right)}, l_{ \pm}=l_{ \pm}^{(1)}+\cdots+l_{ \pm}^{\left(m_{+}\right)}$for $1 \leq i_{r} \leq k_{-}^{(r)}, r=1, \ldots, m_{+}$. It is easy to see that boundary sewing operations or $\gamma_{\left(i_{1}, \ldots, i_{m_{+}}\right)}^{B}$, together with permutation group actions on the order of boundary punctures, provide $\mathbb{S}$ with the structure of a partial dioperad.

There are also so-called interior sewing operations [HKo1,Ko2] on $\mathbb{S}$, denoted as ${ }_{i} \infty_{-j}^{I}$, which sew the $i^{\text {th }}$ positively oriented interior puncture of a disk with the $j^{\text {th }}$ negatively oriented puncture of a sphere. The interior sewing operations define a right action of $\mathbb{K}$ on $\mathbb{S}$ :

$$
\begin{array}{r}
\mathbb{S}\left(m_{-}, m_{+} \mid n_{-}, n_{+}\right) \times \mathbb{K}\left(p_{-}^{(1)}, p_{+}^{(1)}\right) \times \cdots \times \mathbb{K}\left(p_{-}^{\left(n_{+}\right)}, p_{+}^{\left(n_{+}\right)}\right) \\
\stackrel{\gamma_{\left(j_{1}, \ldots, j_{n_{+}}\right)}^{\longrightarrow}}{\longrightarrow} \mathbb{S}\left(m_{-}, m_{+} \mid n_{-}-n_{+}+p_{-}, p_{+}\right),
\end{array}
$$

where $p_{ \pm}=p_{ \pm}^{(1)}+\cdots+p_{ \pm}^{\left(n_{+}\right)}$, for $1 \leq j_{s} \leq p_{-}^{(s)}, s=1, \ldots, n_{+}$. Such action gives $\mathbb{S}$ a right $\mathbb{K}$-module structure.

Let $\gamma^{B}=\left\{\gamma_{\left(i_{1}, \ldots, i_{n}\right)}^{B}\right\}$ and $\gamma^{I}=\left\{\gamma_{\left(i_{1}, \ldots, i_{n}\right)}^{I}\right\}$. The following proposition is clear.

Proposition 2.9. $\left(\mathbb{S} \mid \mathbb{K},\left(\gamma^{B}, \gamma^{I}\right)\right)$ is a $\quad \mathbb{R}_{+} \mid \mathbb{C}^{\times}$-rescalable 2-colored partial dioperad.

We call $\left(\mathbb{S} \mid \mathbb{K},\left(\gamma^{B}, \gamma^{I}\right)\right)$ Swiss-cheese partial dioperad. When it is restricted on $\mathfrak{S}=\{\mathbb{S}(1, m \mid 0, n)\}_{m, n \in \mathbb{N}}$, it is nothing but the so-called Swiss-cheese partial operad [HKo1, Ko2].

In [HKo1,Ko2], we show that the Swiss-cheese partial operad $\mathfrak{S}$ can be naturally embedded into the sphere partial operad $K$ via the so-called doubling map, denoted as $\delta: \mathfrak{S} \hookrightarrow K$. Such doubling map $\delta$ obviously can be extended to a doubling map $\mathbb{S} \hookrightarrow \mathbb{K}$, still denoted as $\delta$. In particular, the general element (2.4) maps under $\delta$ to

$$
\begin{gathered}
\left(\left(z_{-1}, a_{0}^{(-1)}, A^{(-1)}\right), \ldots,\left(z_{-n_{-}}, a_{0}^{\left(-n_{-}\right)}, A^{\left(-n_{-}\right)}\right)\right. \\
\left(\bar{z}_{-1}, \overline{a_{0}^{(-1)}}, \overline{A^{(-1)}}\right), \ldots\left(\bar{z}_{-n_{-}}, \overline{a_{0}^{\left(-n_{-}\right)}}, \overline{A^{\left(-n_{-}\right)}}\right) \\
\left(z_{1}, a_{0}^{(1)}, A^{(1)}\right), \ldots,\left(z_{-1}, b_{0}^{(-1)}, B^{(-1)}\right), \ldots,\left(r_{-m_{-}}, b_{0}^{\left(-m_{-}\right)}, B^{\left(n_{+}\right)}, A^{\left(n_{+}\right)}\right), \\
\left(\bar{z}_{1}, \overline{a_{0}^{(1)}}, \overline{A^{(1)}}\right), \ldots,\left(\bar{z}_{n_{+}}, \overline{a_{0}^{\left(n_{+}\right)}}, \overline{A^{\left(n_{+}\right)}}\right), \\
\left.\left(r_{1}, b_{0}^{(1)}, B^{(1)}\right), \ldots,\left(r_{m_{+}}, b_{0}^{\left(m_{+}\right)}, B^{\left(m_{+}\right)}\right)\right) \mathbb{K} \cdot
\end{gathered}
$$

The following proposition is clear. 
Proposition 2.10. Let $P_{i} \in \mathbb{S}\left(m_{-}^{(i)}, m_{+}^{(i)} \mid n_{-}^{(i)}, n_{+}^{(i)}\right), i=1,2$ and $Q \in \mathbb{K}\left(m_{-}, m_{+}\right)$. If $P_{1 i} \infty_{-j}^{B} P_{2}$ and $P_{1 k} \infty_{-l}^{I} Q$ exists for $1 \leq i \leq m_{+}^{(1)}, 1 \leq j \leq m_{-}^{(2)}$ and $1 \leq k \leq n_{+}^{(1)}$, $1 \leq l \leq m_{-}$, then we have

$$
\begin{aligned}
\delta\left(P_{1}{ }_{i} \infty_{-j}^{B} P_{2}\right) & =\delta\left(P_{1}\right)_{2 n_{+}^{(1)+i}} \infty_{-\left(2 n_{-}^{(2)}+j\right)} \delta\left(P_{2}\right), \\
\delta\left(P_{1}{ }_{k} \infty_{-l}^{I} Q\right) & =\left(\delta\left(P_{1}\right)_{k} \infty_{-l} Q\right)_{n_{+}^{(1)} m_{+-1+k}} \infty_{-l} \bar{Q} .
\end{aligned}
$$

By the above Proposition, we can identify $\mathbb{S}$ as its image under $\delta$ in $\mathbb{K}$ with boundary sewing operations replaced by ordinary sewing operations in $\mathbb{K}$ and interior sewing operations replaced by double-sewing operations in $\mathbb{K}$ as given in (2.11).

The $\mathbb{C}$-extension $\tilde{\mathbb{S}}^{c}\left(m_{-}, m_{+} \mid n_{-}, n_{+}\right)$of $\mathbb{S}\left(m_{-}, m_{+} \mid n_{-}, n_{+}\right)$is defined to be the pullback bundle of $\tilde{\mathbb{K}}^{c}\left(2 n_{-}+m_{-}, 2 n_{+}+m_{+}\right)$. We denote the canonical section on $\tilde{\mathbb{S}}^{c}$, which is induced from that on $\tilde{\mathbb{K}}^{c}$, as $\psi_{\mathbb{S}}$. The boundary (interior) sewing operations can be naturally extended to $\tilde{\mathbb{S}}^{c}$. We denote them as $\tilde{\infty}^{B}\left(\tilde{\infty}^{I}\right)$ and the corresponding substitution maps as $\tilde{\gamma}^{B}\left(\tilde{\gamma}^{I}\right)$. There is a natural right action of $\tilde{\mathbb{K}}^{c} \otimes \overline{\tilde{\mathbb{K}}^{c}}$ on $\tilde{\mathbb{S}}^{c}$ defined by $\tilde{\gamma}^{I}$.

The following proposition is also clear.

Proposition 2.11. $\left(\tilde{\mathbb{S}}^{c} \mid \tilde{\mathbb{K}}^{c} \otimes \overline{\tilde{\mathbb{K}}^{\bar{c}}},\left(\tilde{\gamma}^{B}, \tilde{\gamma}^{I}\right)\right)$ is a $\mathbb{R}_{+} \mid \mathbb{C}^{\times}$-rescalable 2-colored partial dioperad.

We will call the structure $\left(\tilde{\mathbb{S}}^{c} \mid \tilde{\mathbb{K}}^{c} \otimes \overline{\tilde{\mathbb{K}}^{\bar{c}}},\left(\tilde{\gamma}^{B}, \tilde{\gamma}^{I}\right)\right)$ a Swiss-cheese partial dioperad with central charge $c$.

Definition 2.12. An algebra over $\tilde{\mathbb{S}}^{c}$ viewed as a partial dioperad, $(U, v)$, is called smooth if

1. $U=\oplus_{n \in \mathbb{R}} U_{(n)}$ is a completely reducible $\mathbb{R}_{+}$-module, where $r \cdot u=r^{n} u$, $\forall r \in \mathbb{R}_{+}, u \in U_{(m, n)}$.

2. $\operatorname{dim} U_{(n)}<\infty, \forall n \in \mathbb{R}$ and $\operatorname{dim} U_{(n)}=0$ for $n$ sufficiently small.

3. $v$ is linear on fiber and smooth on the base space $\mathbb{S}$.

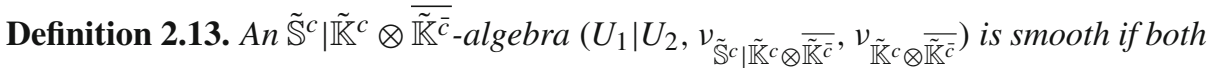
$\left(U_{1}, v_{\tilde{\mathbb{S}}^{c} \mid \tilde{\mathbb{K}}^{c} \otimes} \otimes \tilde{\mathbb{K}}^{\bar{c}}\right)$ and $\left(U_{2}, v_{\tilde{\mathbb{K}}^{c} \otimes} \otimes \tilde{\mathbb{K}}^{\bar{c}}\right)$ as algebras over partial dioperads are smooth.

2.3. Open-closed field algebras over $V$. Let $\left(V_{o p}, Y_{o p}, \iota_{o p}\right)$ be an open-string vertex operator algebra over $V$ and $\left(V_{c l}, \mathbb{Y}, \iota_{c l}\right)$ a conformal full field algebra over $V \otimes V$. Let

$$
\left(\left(V_{c l}, \mathbb{Y}, \iota_{c l}\right),\left(V_{o p}, Y_{o p}, \iota_{o p}\right), \mathbb{Y}_{c l-o p}\right)
$$

be an open-closed field algebra over $V$ [Ko2]. We denote the formal vertex operators associated with $Y_{o p}$ and $\mathbb{Y}$ as $Y_{o p}^{f}$ and $\mathbb{Y}^{f}$ respectively. Let $\omega^{L}=\iota_{c l}(\omega \otimes \mathbf{1}), \omega^{R}=\iota_{c l}(\mathbf{1} \otimes \omega)$ and $\omega_{o p}=\iota_{o p}(\omega)$. We have

$$
\begin{aligned}
\mathbb{Y}^{f}\left(\omega^{L} ; x, \bar{x}\right) & =\mathbb{Y}^{f}\left(\omega^{L}, x\right)=\sum_{n \in \mathbb{Z}} L(n) \otimes 1 x^{-n-2}, \\
\mathbb{Y}^{f}\left(\omega^{R} ; x, \bar{x}\right) & =\mathbb{Y}^{f}\left(\omega^{R}, \bar{x}\right)=\sum_{n \in \mathbb{Z}} 1 \otimes L(n) \bar{x}^{-n-2}, \\
Y_{o p}^{f}\left(\omega_{o p}, x\right) & =\sum_{n \in \mathbb{Z}} L(n) x^{-n-2} .
\end{aligned}
$$


We also set $L^{L}(n)=L(n) \otimes 1$ and $L^{R}(n)=1 \otimes L(n)$ for $n \in \mathbb{Z}$.

For $u \in V$, we showed in [Ko2] that $\mathbb{Y}_{c l-o p}\left(\iota_{c l}(u \otimes \mathbf{1}) ; z, \bar{z}\right)$ and $\mathbb{Y}_{c l-o p}\left(\iota_{c l}(\mathbf{1} \otimes\right.$ $u) ; z, \bar{z})$ are holomorphic and antiholomorphic respectively. So we also denote them simply by $\mathbb{Y}_{c l-o p}\left(\iota_{c l}(u \otimes \mathbf{1}), z\right)$ and $\mathbb{Y}_{c l-o p}\left(\iota_{c l}(\mathbf{1} \otimes u), \bar{z}\right)$ respectively.

By the $V$-invariant boundary condition [Ko2], we have

$$
\mathbb{Y}_{c l-o p}\left(\omega^{L}, r\right)=Y_{o p}\left(\omega_{o p}, r\right)=\mathbb{Y}_{c l-o p}\left(\omega^{R}, r\right) .
$$

We assume that both $V_{o p}$ and $V_{c l}$ are equipped with nondegenerate invariant bilinear forms $(\cdot, \cdot)_{o p}$ and $(\cdot, \cdot)_{c l}$ respectively.

Lemma 2.14. For any $u \in V_{c l}$ and $v_{1}, v_{2} \in V_{o p}$ and $z \in \mathbb{H}$, we have

$$
\begin{aligned}
& \left(v_{2}, \mathbb{Y}_{c l-o p}(u ; z, \bar{z}) v_{1}\right)_{o p} \\
& \quad=\left(\mathbb{Y}_{c l-o p}\left(e^{-z L(1)} z^{-2 L(0)} \otimes e^{-\bar{z} L(1)} \bar{z}^{-2 L(0)} u ;-z^{-1},-\bar{z}^{-1}\right) v_{2}, v_{1}\right)_{o p} .
\end{aligned}
$$

Proof. Using (1.26), for fixed $z \in \mathbb{H}$, we have,

$$
\begin{aligned}
\left(v_{2}, \mathbb{Y}_{c l-o p}(u ; z, \bar{z}) v_{1}\right)_{o p} \\
\quad=\left(v_{2}, \mathbb{Y}_{c l-o p}(u ; z, \bar{z}) Y_{o p}(\mathbf{1}, r) v_{1}\right)_{o p} \\
\quad=\left(v_{2}, Y_{o p}\left(\mathbb{Y}_{c l-o p}(u ; z-r, \bar{z}-r) \mathbf{1}, r\right) v_{1}\right)_{o p} \\
\quad=\left(e^{-r^{-1} L(-1)} Y_{o p}\left(v_{2}, r^{-1}\right) e^{-r L(1)} r^{-2 L(0)} \mathbb{Y}_{c l-o p}(u ; z-r, \bar{z}-r) \mathbf{1}, v_{1}\right)_{o p}
\end{aligned}
$$

for $|z|>r>|z-r|>0$. Notice that $e^{-r^{-1} L(-1)} \in \operatorname{Aut}\left(\bar{V}_{o p}\right), \forall r \in \mathbb{C}$. By taking $v_{2}=\mathbf{1}_{o p}$, it is easy to see that

$$
e^{-r L(1)} r^{-2 L(0)} \mathbb{Y}_{c l-o p}(u ; z-r, \bar{z}-r) \mathbf{1}
$$

is a well-defined element in $\bar{V}_{o p}$ for $|z|>r>|z-r|>0$. Because of the chirality splitting property of $\mathbb{Y}_{c l-o p}$ (see (1.72), (1.73) in [Ko2]), it is easy to show that (2.17) equals

$$
\mathbb{Y}_{c l-o p}\left(e^{-z L(1)} z^{-2 L(0)} \otimes e^{-\bar{z} L(1)} \bar{z}^{-2 L(0)} u, r^{-1}-z^{-1}, r^{-1}-\bar{z}^{-1}\right) \mathbf{1}
$$

for $r>|z-r|>0$. By the commutativity I of the analytic open-closed field algebra proved in [Ko2], we know that for fixed $z \in \mathbb{H}$,

$$
\begin{array}{r}
e^{-r^{-1} L(-1)} Y_{o p}\left(v_{2}, r^{-1}\right) \mathbb{Y}_{c l-o p}\left(e^{-z L(1)} z^{-2 L(0)}\right. \\
\left.\otimes e^{-\bar{z} L(1)} \bar{z}^{-2 L(0)} u, r^{-1}-z^{-1}, r^{-1}-\bar{z}^{-1}\right) \mathbf{1}
\end{array}
$$

and

$$
\begin{aligned}
e^{-r^{-1} L(-1)} \mathbb{Y}_{c l-o p}\left(e^{-z L(1)} z^{-2 L(0)}\right. & \\
& \left.\quad \otimes e^{-\bar{z} L(1)} \bar{z}^{-2 L(0)} u, r^{-1}-z^{-1}, r^{-1}-\bar{z}^{-1}\right) Y_{o p}\left(v_{2}, r^{-1}\right) \mathbf{1} \\
= & e^{-r^{-1} L(-1)} \mathbb{Y}_{c l-o p}\left(e^{-z L(1)} z^{-2 L(0)}\right. \\
& \left.\otimes e^{-\bar{z} L(1)} \bar{z}^{-2 L(0)} u, r^{-1}-z^{-1}, r^{-1}-\bar{z}^{-1}\right) e^{r^{-1} L(-1)} v_{2}
\end{aligned}
$$


converge in different domains for $r$, but are an analytic continuation of each other along a path in $r \in \mathbb{R}_{+}$. Moreover, using the $L(-1)$ property of the intertwining operator and chirality splitting property of $\mathbb{Y}_{c l-o p}$ again, the right hand side of (2.19) equals

$$
\mathbb{Y}_{c l-o p}\left(e^{-z L(1)} z^{-2 L(0)} \otimes e^{-\bar{z} L(1)} \bar{z}^{-2 L(0)} u,-z^{-1},-\bar{z}^{-1}\right) v_{2}
$$

for $\left|r^{-1}-z^{-1}\right|>\left|r^{-1}\right|$. Therefore, both sides of (2.15) as constant functions of $r$ are analytic continuation of each other. Hence (2.15) must hold identically for all $z \in \mathbb{H}$. $\square$

For $u_{1}, \ldots, u_{l} \in V_{c l}, v_{1}, \ldots, v_{n} \in V_{c l}, r_{1}, \ldots, r_{n} \in \mathbb{R}, r_{1}>\cdots>r_{n}$ and $z_{1}, \ldots, z_{l} \in \mathbb{H}$, we define

$$
\begin{gathered}
m_{c l-o p}^{(l ; n)}\left(u_{1}, \ldots, u_{l} ; v_{1}, \ldots, v_{n} ; z_{1}, \bar{z}_{1}, \ldots, z_{l}, \bar{z}_{l} ; r_{1}, \ldots, r_{n}\right) \\
:=e^{-r_{n} L(-1)} m_{c l-o p}^{(l ; n)}\left(u_{1}, \ldots, u_{l} ; v_{1}, \ldots, v_{n} ; z_{1}-r_{n}, \bar{z}_{1}-r_{n}, \ldots,\right. \\
\left.z_{l}-r_{n}, \bar{z}_{l}-r_{n} ; r_{1}-r_{n}, \ldots, r_{n-1}-r_{n}, 0\right)
\end{gathered}
$$

We simply extend the definition of $m_{c l-o p}^{(l ; n)}$ to a domain where some of $r_{i}$ can be negative. Note that such a definition is compatible with $L(-1)$-properties of $m_{c l-o p}$.

Lemma 2.15. For $u_{1}, \ldots, u_{l} \in V_{c l}, v, v_{1}, \ldots, v_{n} \in V_{c l}, r_{1}, \ldots, r_{n} \in \mathbb{R}, r_{1}>\cdots>$ $r_{n}=0$ and $z_{1}, \ldots, z_{l} \in \mathbb{H}$,

$$
\begin{aligned}
& \left(v, m_{c l-o p}^{(l ; n)}\left(u_{1}, \ldots, u_{l} ; v_{1}, \ldots, v_{n} ; z_{1}, \bar{z}_{1}, \ldots, z_{l}, \bar{z}_{l} ; r_{1}, \ldots, r_{n}\right)\right)_{o p} \\
& =\left(v_{n}, m_{c l-o p}^{(l ; n)}\left(F_{1} u_{1}, \ldots, F_{l} u_{l} ; v, G_{1} v_{1}, \ldots, G_{n-1} v_{n-1} ;\right.\right. \\
& \left.\left.\quad-z_{1}^{-1},-\bar{z}_{1}^{-1}, \ldots,-z_{l}^{-1},-\bar{z}_{l}^{-1} ; 0,-r_{1}^{-1}, \ldots,-r_{n-1}^{-1}\right)\right)_{o p},
\end{aligned}
$$

where

$$
\begin{aligned}
F_{i} & =e^{-z_{i} L(1)} z_{i}^{-2 L(0)} \otimes e^{-\bar{z}_{i} L(1)} \bar{z}_{i}^{-2 L(0)}, \quad i=1, \ldots, l, \\
G_{j} & =e^{-r_{j} L(1)} r_{j}^{-2 L(0)}, \quad j=1, \ldots, n-1 .
\end{aligned}
$$

Proof. By Lemma 2.14, (2.21) is clearly true for $l=0,1 ; n=0,1$. By (1.26) and (1.27), (2.21) is true for $l=0, n=2$. We then prove the lemma by induction. Assume that (2.21) is true for $l=k \geq 0, n=m \geq 2$ or $l=k \geq 1, n=m \geq 1$.

Let $l=k$ and $n=m+1$. It is harmless to assume that $0<r_{n-1},\left|z_{i}\right|<r_{n-2}$, $i=l_{1}+1, \ldots, l$ for some $l_{1} \leq l$. Using the induction hypothesis, we obtain

$$
\begin{aligned}
(v, & \left.m_{c l-o p}^{(l ; n)}\left(u_{1}, \ldots, u_{l} ; v_{1}, \ldots, v_{n} ; z_{1}, \bar{z}_{1}, \ldots, z_{l}, \bar{z}_{l} ; r_{1}, \ldots, r_{n}\right)\right)_{o p} \\
= & \left(v, m_{c l-o p}^{\left(l_{1} ; n-1\right)}\left(u_{1}, \ldots, u_{l_{1}} ; v_{1}, \ldots, v_{n-2}, m_{c l-o p}^{\left(l-l_{1} ; 2\right)}\left(u_{l_{1}+1}, \ldots, u_{l} ; v_{n-1}, v_{n} ;\right.\right.\right. \\
& \left.\left.\left.z_{l_{1}+1}, \bar{z}_{l_{1}+1}, \ldots, z_{l}, \bar{z}_{l} ; r_{n-1}, 0\right) ; z_{1}, \bar{z}_{1}, \ldots, z_{l_{1}}, \bar{z}_{l_{1}} ; r_{1}, \ldots, r_{n-2}, 0\right)\right)_{o p} \\
= & \left(m _ { c l - o p } ^ { ( l _ { 1 } ; n - 1 ) } \left(F_{1} u_{1}, \ldots, F_{l_{1}} u_{l_{1}} ; v, G_{1} v_{1}, \ldots, G_{n-1} v_{n-1} ;\right.\right. \\
& \left.-z_{1}^{-1},-\bar{z}_{1}^{-1}, \ldots,-z_{l_{1}}^{-1},-\bar{z}_{l_{1}}^{-1} ; 0,-r_{1}^{-1}, \ldots,-r_{n-1}^{-1}\right), \\
& \left.m_{c l-o p}^{\left(l-l_{1} ; 2\right)}\left(u_{l_{1}+1}, \ldots, u_{l} ; v_{n-1}, v_{n} ; z_{l_{1}+1}, \bar{z}_{l_{1}+1}, \ldots, z_{l}, \bar{z}_{l} ; r_{n-1}, 0\right)\right)_{o p} \\
= & \left(m _ { c l - o p } ^ { ( l - l _ { 1 } ; 2 ) } \left(F_{l_{1}+1} u_{l_{1}+1}, \ldots, F_{l} u_{l} ; m_{c l-o p}^{\left(l_{1} ; n-1\right)}\left(F_{1} u_{1}, \ldots, F_{l_{1}} u_{l_{1}} ;\right.\right.\right.
\end{aligned}
$$




$$
\begin{aligned}
& \left.v, G_{1} v_{1}, \ldots, G_{n-1} v_{n-1} ;-z_{1}^{-1},-\bar{z}_{1}^{-1}, \ldots,-z_{l_{1}}^{-1},-\bar{z}_{l_{1}}^{-1} ; 0,-r_{1}^{-1}, \ldots,-r_{n-2}^{-1}\right), \\
& \left.\left.G_{n-1} v_{n-1} ;-z_{l_{1}+1}^{-1},-\bar{z}_{l_{1}+1}^{-1}, \ldots,-z_{l}^{-1},-\bar{z}_{l}^{-1} ; 0,-r_{n-1}^{-1}\right), v_{n}\right)_{o p} \\
= & \sum_{s \in \mathbb{R}}\left(e ^ { - r _ { n - 1 } ^ { - 1 } L ( - 1 ) } m _ { c l - o p } ^ { ( l - l _ { 1 } ; 2 ) } \left(F_{l_{1}+1} u_{l_{1}+1}, \ldots, F_{l} u_{l} ; P_{s} e^{-r_{n-2}^{-1} L(-1)} m_{c l-o p}^{\left(l-l_{1} ; n-1\right)}\left(F_{1} u_{1},\right.\right.\right. \\
& \ldots, F_{l_{1}} u_{l_{1}} ; v, G_{1} v_{1}, \ldots, G_{n-1} v_{n-1} ;-z_{1}^{-1}+r_{n-2}^{-1},-\bar{z}_{1}^{-1}+r_{n-2}^{-1}, \\
& \left.\ldots,-z_{l_{1}}^{-1}+r_{n-2}^{-1},-\bar{z}_{l_{1}}^{-1}+r_{n-2}^{-1} ; r_{n-2}^{-1},-r_{1}^{-1}+r_{n-2}^{-1}, \ldots,-r_{n-3}^{-1}+r_{n-2}^{-1}, 0\right), \\
& G_{n-1} v_{n-1} ;-z_{l_{1}+1}^{-1}+r_{n-1}^{-1},-\bar{z}_{l_{1}+1}^{-1}+r_{n-1}^{-1}, \\
& \left.\left.\ldots,-z_{l}^{-1}+r_{n-1}^{-1},-\bar{z}_{l}^{-1}+r_{n-1}^{-1} ; r_{n-1}^{-1}, 0\right), v_{n}\right)_{o p} .
\end{aligned}
$$

Note that the position of $P_{s}$ and $e^{-r_{n-2}^{-1} L(-1)}$ can not be exchanged in general. Because if we exchange their position, the sum may not converge and then the associativity law does not hold. We want to use analytic continuation to move it to a domain such that we can freely apply the associativity law. By our assumption on $V$, both sides of (2.23) are restrictions of the analytic function of the $z_{l_{1}+1}, \zeta_{l_{1}+1}, \ldots, z_{l}, \zeta_{l}, r_{n-1}$ on $\zeta_{l_{1}+1}=\bar{z}_{l_{1}+1}, \ldots, \zeta_{l}=\bar{z}_{l}$. Let $\tilde{z}_{l_{1}+1}, \ldots, \tilde{z}_{l}, \tilde{r}_{n-1}$ satisfy the following conditions:

$$
\left|-\tilde{z}_{p}^{-1}+r_{n-2}^{-1}\right|, \tilde{r}_{n-1}^{-1}-r_{n-2}^{-1}>\left|-z_{i}^{-1}+r_{n-2}^{-1}\right|, r_{n-2}^{-1},-r_{j}^{-1}+r_{n-2}^{-1},
$$

for all $i=1, \ldots, l, j=1, \ldots, n-3$ and $p=l_{1}+1, \ldots, l$. Note that such a condition define a nonempty open subset on $\mathbb{H}^{l} \times \mathbb{R}_{+}$. Choose a path $\gamma_{1}$ in the complement of the diagonal in $\mathbb{H}^{l}$ from initial point $\left(z_{l_{1}+1}, \ldots, z_{l}\right)$ to $\left(\tilde{z}_{l_{1}+1}, \ldots, \tilde{z}_{l}\right)$ and a path $\gamma_{2}$ in $\mathbb{R}_{+}$ from $r_{n-1}$ to $\tilde{r}_{n-1}$. We also denote the complex conjugate of path $\gamma_{1}$ as $\bar{\gamma}_{1}$, which is a path in $\overline{\mathbb{H}}^{l}$. Combine $\gamma_{1}, \bar{\gamma}_{1}$ with $\gamma_{2}$, we obtain a path $\gamma$ from $\left(z_{l_{1}+1}, \bar{z}_{l_{1}+1}, \ldots, z_{l}, \bar{z}_{l}, r_{n-1}\right)$ to $\left(\tilde{z}_{l_{1}+1}, \overline{\tilde{z}}_{l_{1}+1}, \ldots, \tilde{z}_{l}, \overline{\tilde{z}}_{l}, \tilde{r}_{n-1}\right)$. Analytically continuing the right hand side of $(2.23)$ along the path $\gamma$, we obtain, by the properties of $m_{c l-o p}^{(l ; n)}[\mathrm{Ko} 2]$,

$$
\begin{aligned}
& \left(e ^ { - \tilde { r } _ { n - 1 } ^ { - 1 } L ( - 1 ) } m _ { c l - o p } ^ { ( l - l _ { 1 } ; 2 ) } \left(F_{l_{1}+1} u_{l_{1}+1}, \ldots, F_{l} u_{l} ; P_{s} m_{c l-o p}^{\left(l-l_{1} ; n-1\right)}\left(F_{1} u_{1}, \ldots, F_{l_{1}} u_{l_{1}}\right.\right.\right. \\
& \quad v, G_{1} v_{1}, \ldots, G_{n-1} v_{n-1} ;-z_{1}^{-1}+r_{n-2}^{-1},-\bar{z}_{1}^{-1}+r_{n-2}^{-1}, \\
& \left.\quad \ldots,-z_{l_{1}}^{-1}+r_{n-2}^{-1},-\bar{z}_{l_{1}}^{-1}+r_{n-2}^{-1} ; r_{n-2}^{-1},-r_{1}^{-1}+r_{n-2}^{-1}, \ldots,-r_{n-3}^{-1}+r_{n-2}^{-1}, 0\right) \\
& \tilde{G}_{n-1} v_{n-1} ;-\tilde{z}_{l_{1}+1}^{-1}+r_{n-1}^{-1},-\overline{\tilde{z}}_{l_{1}+1}^{-1}+r_{n-1}^{-1}, \\
& \left.\left.\quad \ldots,-\tilde{z}_{l}^{-1}+r_{n-1}^{-1},-\overline{\tilde{z}}_{l}^{-1}+r_{n-1}^{-1} ; \tilde{r}_{n-1}^{-1}-r_{n-2}^{-1}, 0\right), v_{n}\right)_{o p}
\end{aligned}
$$

where $\tilde{G}_{n-1}=e^{-\tilde{r}_{n-1} L(1)} \tilde{r}_{n-1}^{-2 L(0)}$. Using the associativity of open-closed field algebra and $L(-1)$-properties of $m_{c l-o p}$, we see that (2.25) further equals

$$
\begin{aligned}
& \left(m _ { c l - o p } ^ { ( l ; n + 1 ) } \left(F_{1} u_{1}, \ldots, F_{l} u_{l} ; v, G_{1} v_{1}, \ldots, \tilde{G}_{n-1} v_{n-1} ;-z_{1}^{-1},-\bar{z}_{1}^{-1}, \ldots,\right.\right. \\
& \left.\left.\quad-z_{l_{1}}^{-1},-\bar{z}_{l_{1}}^{-1},-\tilde{z}_{l_{1}+1}^{-1},-\overline{\tilde{z}}_{l_{1}+1}^{-1}, \ldots,-z_{l}^{-1},-\bar{z}_{l}^{-1} ; 0,-r_{1}^{-1}, \ldots,-\tilde{r}_{n-1}^{-1}\right), v_{n}\right)_{o p} .
\end{aligned}
$$

By analytically continuing (2.26) along the path $-\gamma$, which is $\gamma$ reversed, we obtain the right hand side of (2.21). Hence (2.23) and the right hand side of (2.21) are analytic continuation of each other along path $(-\gamma) \circ(\gamma)$ which is a constant path. Hence (2.21) holds for $l=k, n=m+1$. 
Now let $l=k+1, n=m$. The proof is similar to the case $l=k, n=m+1$. We only point out the difference. Using the smoothness of $m_{c l-o p}$, it is enough to prove the case when $\left|z_{i}\right| \neq\left|z_{j}\right|$ for $i, j=1, \ldots, l$ and $i \neq j$. Without losing generality, we assume that $\left|z_{1}\right|>\cdots>\left|z_{l}\right|>0$. Let $n_{1} \leq n$ be the smallest so that $0<r_{j}<\left|z_{l}\right|$ for $j \geq n_{1}$. Then we have

$$
\begin{aligned}
& \left(v, m_{c l-o p}^{(l ; n)}\left(u_{1}, \ldots, u_{l} ; v_{1}, \ldots, v_{n} ; z_{1}, \bar{z}_{1}, \ldots, z_{l}, \bar{z}_{l} ; r_{1}, \ldots, r_{n}\right)\right)_{o p} \\
& =\left(v, m_{c l-o p}^{\left(l-1 ; n_{1}\right)}\left(u_{1}, \ldots, u_{l-1} ; v_{1}, \ldots, v_{n_{1}-1}, m_{c l-o p}^{\left(1 ; n-n_{1}+1\right)}\left(u_{l} ; v_{n_{1}}, \ldots, v_{n} ;\right.\right.\right. \\
& \left.\left.\left.\quad z_{l}, \bar{z}_{l} ; r_{n_{1}}, \ldots, r_{n}\right) ; z_{1}, \bar{z}_{1}, \ldots, z_{l-1}, \bar{z}_{l-1} ; r_{1}, \ldots, r_{n_{1}-1}\right)\right)_{o p} .
\end{aligned}
$$

We can then apply (2.21) as in (2.23) for the case $l \leq k, n \leq m$, which is true by our induction hypothesis. The rest of the proof is entirely the same as that of the case $l=k, n=m+1$.

We define a map, for $z, \zeta \in \mathbb{C}$ and $z \neq \zeta, \iota_{c l-o p}(z, \zeta): V_{c l} \rightarrow \bar{V}_{o p}$ as

$$
\iota_{c l-o p}(z, \zeta)(u)=\mathbb{Y}_{c l-o p}(u ; z, \zeta) \mathbf{1}_{o p} .
$$

We denote its adjoint as $\iota_{c l-o p}^{*}(z, \zeta)$. Namely, $\iota_{c l-o p}^{*}(z, \zeta): V_{o p} \rightarrow \overline{V_{c l}}$ is given by

$$
\left(\iota_{c l-o p}^{*}(z, \zeta)(w), u\right)_{c l}=\left(w, \iota_{c l-o p}(z, \zeta)(u)\right)_{o p}
$$

for any $u \in V_{c l}$ and $w \in V_{o p}$.

Let $Q$ be an element in $\mathcal{T}_{\mathbb{S}}\left(n_{-}, n_{+} \mid m_{-}, m_{+}\right)$of form (2.4). Let $\alpha$ be the map (1.30) so that $s_{1}, \ldots, s_{n_{-}+n_{+}}$, defined as $s_{i}:=r_{\alpha^{-1}(i)}$, satisfy $\infty \geq s_{1}>\cdots>s_{n_{-}+n_{+}} \geq 0$. Then we define

$$
v_{c l-o p}\left(\lambda \psi_{\mathbb{S}}(Q)\right)\left(u_{1} \otimes \cdots \otimes u_{m_{+}} \otimes v_{1} \otimes \cdots \otimes v_{n_{+}}\right)
$$

as follows:

1. If $s_{1} \neq \infty,(2.29)$ is given by

$$
\begin{gathered}
\lambda \sum_{i_{1}, \ldots, i_{m_{-}} ; j_{1}, \ldots, j_{n_{-}}}\left(\mathbf{1}_{o p}, m_{c l-o p}^{\left(m_{-}+m_{+} ; n_{-}+n_{+}\right)}\left(u_{-1}, \ldots, u_{-m_{-}}, u_{1}, \ldots, u_{m_{+}}\right.\right. \\
w_{1}, \ldots, w_{n_{-}+n_{+}} ; z_{-1}, \bar{z}_{-1}, \ldots, z_{-m_{-}}, \bar{z}_{-m_{-}}, z_{1}, \bar{z}_{1}, \ldots, z_{m_{+}}, \bar{z}_{m_{+}} \\
\left.\left.s_{1}, \ldots, s_{n_{-}+n_{+}}\right)\right)_{o p} \cdot e^{i_{1}} \otimes \cdots \otimes e^{i_{n_{-}}} \otimes f^{j_{1}} \otimes \cdots \otimes f^{j_{m_{-}}}
\end{gathered}
$$

where

$$
\begin{aligned}
u_{p} & =e^{-L_{+}^{L}\left(A^{(p)}\right)-L_{+}^{R}\left(\overline{A^{(p)}}\right)}\left(a_{0}^{(p)}\right)^{-L^{L}(0) \overline{a_{0}^{(p)}}-L^{R}(0)} e_{i_{-p},}, \\
u_{q} & =e^{\left.-L_{+}^{L}\left(A^{(q)}\right)-L_{+}^{R} \overline{A^{(q)}}\right)}\left(a_{0}^{(q)}\right)^{-L^{L}(0) \overline{a_{0}^{(q)}}-L^{R}(0)} u_{q}, \\
w_{\alpha(k)} & =e^{-L_{+}\left(B^{(k)}\right)}\left(b_{0}^{(k)}\right)^{-L(0)} f_{j_{-k}}, \\
w_{\alpha(l)} & =e^{-L_{+}\left(B^{(l)}\right)}\left(b_{0}^{(l)}\right)^{-L(0)} v_{l},
\end{aligned}
$$

for $p=-1, \ldots,-n_{-}, q=1, \ldots, n_{+}, k=-1, \ldots,-m_{-}$and $l=1, \ldots, m_{+}$.

2. When $r_{k}=\infty$ for some $k=-m_{-}, \ldots,-1,1, \ldots, m_{+}$. Equation (2.29) is given by the formula obtained from (2.30) by exchanging $\mathbf{1}_{o p}$ with $w_{\alpha(k)}$. 
Lemma 2.16. $v_{c l-o p}$ is $S L(2, \mathbb{R})$-invariant.

Proof. The $S L(2, \mathbb{R})$ is generated by the following three transformations $1 . w \mapsto$ $a w, \forall a \in \mathbb{R}^{+} ; 2 . w \mapsto w-b, \forall b \in \mathbb{R} ; 3 . w \mapsto \frac{-1}{w}$. That $v_{c l-o p}$ is invariant under the first two transformations simply follows from the $L(0)$ - and $L(-1)$-properties of $m_{c l-o p}$. That $v_{c l-o p}$ is invariant under the third transformation is proved in Lemma 2.15.

Hence $v_{c l-o p}$ induces a map $\tilde{\mathbb{S}}^{c} \rightarrow \mathbb{E}_{V_{o p} \mid V_{c l}}^{\mathbb{R}_{+} \mid \mathbb{C}^{\times}}$, which is still denoted as $v_{c l-o p}$. We list a few interesting cases:

$$
\begin{aligned}
& v_{c l-o p}\left(\psi_{\mathbb{S}}\left([((\infty, 1, \mathbf{0}) \mid) \|(\mid(z, 1, \mathbf{0}))]_{\mathbb{S}}\right)\right)=\iota_{c l-o p}(z, \bar{z}), \\
& v_{c l-o p}\left(\psi_{\mathbb{S}}\left([(\mid(\infty, 1, \mathbf{0})) \|((z, 1, \mathbf{0}) \mid)]_{\mathbb{S}}\right)\right)=\iota^{*}(z, \bar{z}),
\end{aligned}
$$

and for $b \in \mathbb{R}_{+}, B \in \mathbb{R}^{\infty}, a \in \mathbb{C}^{\times}, A \in \mathbb{C}^{\infty}$ and $v \in V_{\text {op }}$, we have

$$
\begin{aligned}
& v_{c l-o p}\left(\psi_{\mathbb{S}}\left([(\mid(\infty, b, B)) \|((z, a, A) \mid)]_{\mathbb{S}}\right)\right) \\
& =a^{-L^{L}(0)} \bar{a}^{-L^{R}(0)} e^{-\sum_{j=1}^{\infty}(-1)^{j}\left[A_{j} L^{L}(-j)+\overline{A_{j}} L^{R}(-j)\right]} \iota^{*}(z, \bar{z})\left(e^{-L_{+}(B)} b^{-L(0)} v\right) .
\end{aligned}
$$

Theorem 2.17. $\left(V_{o p} \mid V_{c l}, v_{c l-o p}, v_{c l}\right)$ is an $\mathbb{R}_{+} \mid \mathbb{C}^{\times}$-rescalable smooth $\tilde{\mathbb{S}}^{c} \mid \tilde{\mathbb{K}}^{c} \otimes$ $\overline{\mathbb{K}^{\bar{c}}}$-algebra.

Proof. The smoothness is automatic. We showed in [Ko2] that $\left(V_{o p} \mid V_{c l}, v_{c l-o p}, v_{c l}\right)$ is an $\mathbb{R}_{+} \mid \mathbb{C}^{\times}$-rescalable smooth $\tilde{\mathfrak{S}}^{c} \mid \tilde{K}^{c} \otimes \bar{K}^{\bar{c}}$-algebra. The rest of the proof is similar to that of Theorem 1.12 in [Ko1]. We omit the detail here.

2.4. Ishibashi states. As we mentioned in the introduction, an open-closed field algebra over $V$ equipped with nondegenerate invariant bilinear forms for both open theory and closed theory contains all the data needed to grow to an open-closed partial field theory of all genus. Without adding more compatibility conditions, itself is already an interesting object to study. We show in this subsection that the famous "Ishibashi states" [I] can be studied in the framework of such an algebra. Throughout this subsection, we fix an open-closed algebra over $V$ given in (2.12) and equipped with nondegenerate invariant bilinear forms $(\cdot, \cdot)_{c l}$ and $(\cdot, \cdot)_{o p}$.

For $u \in V_{o p}$ and $z_{0} \in \mathbb{H}$, we define the boundary state $B_{z_{0}}(u) \in \overline{V_{c l}}$ associated with $u$ and $z_{0}$ by

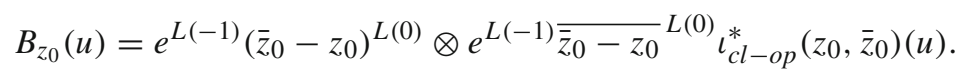

Proposition 2.18. If $u \in V_{o p}$ is a vacuum-like vector [LL], i.e. $L(-1) u=0$, then, for $z_{0} \in \mathbb{H}, B_{z_{0}}(u)$ is an Ishibashi state, i.e.

$$
\left(L^{L}(n)-L^{R}(-n)\right) B_{z_{0}}(u)=0, \quad \forall n \in \mathbb{Z} .
$$

Proof. For $v \in V_{c l}$, the following two functions of $z$ :

$$
\begin{aligned}
& \left(u, \quad \mathbb{Y}_{c l-o p}\left(\omega^{L}, z+z_{0}\right) \mathbb{Y}\left(v ; z_{0}, \bar{z}_{0}\right) \mathbf{1}_{o p}\right)_{o p}, \\
& \left(u, \quad \mathbb{Y}_{c l-o p}\left(\omega^{R} ; \overline{z+z_{0}}\right) \mathbb{Y}\left(v ; z_{0}, \bar{z}_{0}\right) \mathbf{1}_{o p}\right)_{o p}
\end{aligned}
$$


can be extended to a holomorphic function and an antiholomorphic function in $\left\{z \mid z+z_{0} \in\right.$ $\mathbb{H}, z \neq 0\}$ respectively by our assumption on $V$. We denote the extended functions by $g_{1}\left(\omega^{L}, z\right)$ and $g_{2}\left(\omega^{R}, \bar{z}\right)$ respectively.

The following two limits:

$$
\begin{aligned}
& \lim _{z+z_{0} \rightarrow r}\left(1-\frac{z}{\bar{z}_{0}-z_{0}}\right)^{4} g_{1}\left(\omega^{L}, z\right), \\
& \lim _{z+z_{0} \rightarrow r}\left(1-\frac{z}{\bar{z}_{0}-z_{0}}\right)^{4} g_{2}\left(\omega^{R}, \bar{z}\right)
\end{aligned}
$$

exist for all $r \in \mathbb{R}$. Using (2.15), it is easy to see that the above two limits also exist for $r=\infty \in \hat{\mathbb{R}}$ if and only if $u$ is vacuum-like. Hence, by $V$-invariant (or conformal invariant) boundary condition, we have

$$
\lim _{z+z_{0} \rightarrow r}\left(1-\frac{z}{\bar{z}_{0}-z_{0}}\right)^{4} g_{1}\left(\omega^{L}, z\right)=\lim _{z+z_{0} \rightarrow r}\left(1-\frac{z}{\bar{z}_{0}-z_{0}}\right)^{4} g_{2}\left(\omega^{R}, z\right)
$$

for all $r \in \hat{\mathbb{R}}$ when $L(-1) u=0$.

On the other hand, for $\left|z+z_{0}\right|>\left|z_{0}\right|$, we have

$$
\begin{aligned}
& \left(u, \mathbb{Y}_{c l-o p}\left(\omega^{L}, z+z_{0}\right) \mathbb{Y}_{c l-o p}\left(v ; z_{0}, \bar{z}_{0}\right) \mathbf{1}_{o p}\right)_{o p} \\
& =\left(u, \mathbb{Y}_{c l-o p}\left(\mathbb{Y}\left(\omega^{L}, z\right) v ; z_{0}, \bar{z}_{0}\right)_{o p} \mathbf{1}_{o p}\right)_{o p} \\
& =\left(u, \iota_{c l-o p}\left(z_{0}, \bar{z}_{0}\right)\left(\mathbb{Y}\left(\omega^{L}, z\right) v\right)\right)_{o p} \\
& =\left(\iota_{c l-o p}^{*}\left(z_{0}, \bar{z}_{0}\right)(u), \mathbb{Y}\left(\omega^{L}, z\right) v\right)_{c l}
\end{aligned}
$$

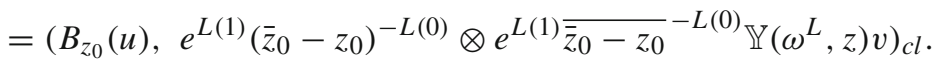

Note that one should check the convergence property of each step in (2.39). In particular, in the last step, the convergence and equality follow from the convergence of early steps and the fact that $\left(\bar{z}_{0}-z_{0}\right)^{-L(0)} e^{-L(-1)} \otimes{\overline{z_{0}-z_{0}}}^{-L(0)} e^{-L(-1)} \in$ Aut $\overline{V_{c l}}$. For $0<|z|<\left|\operatorname{Im} z_{0}\right|$, it is easy to show that

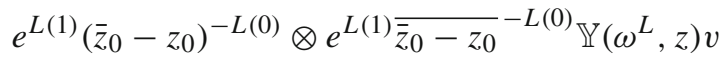

$$
\begin{aligned}
& =\mathbb{Y}\left(e^{\left(1-\frac{z}{\bar{z}_{0}-z_{0}}\right) L^{L}(1)}\left(1-\frac{z}{\bar{z}_{0}-z_{0}}\right)^{-2 L^{L}(0)} .\right. \\
& \left.\cdot\left(\bar{z}_{0}-z_{0}\right)^{-L^{L}(0)} \omega^{L}, \frac{1}{\bar{z}_{0}-z_{0}} \frac{z}{1-\frac{z}{\bar{z}_{0}-z_{0}}}\right) v_{1} \\
& =\left(1-\frac{z}{\bar{z}_{0}-z_{0}}\right)^{-4}\left(\bar{z}_{0}-z_{0}\right)^{-2} \mathbb{Y}\left(\omega^{L}, \frac{1}{\bar{z}_{0}-z_{0}} \frac{z}{1-\frac{z}{\bar{z}_{0}-z_{0}}}\right) v_{1} \text {, }
\end{aligned}
$$

where $v_{1}=e^{L(1)}\left(\bar{z}_{0}-z_{0}\right)^{-L(0)} \otimes e^{L(1)}{\overline{z_{0}}-z_{0}}^{-L(0)} v$. Hence, for all $0<|z|<\left|\operatorname{Im} z_{0}\right|$ and $\left|z+z_{0}\right|>\left|z_{0}\right|$, we obtain

$$
\begin{aligned}
& \left(u, \mathbb{Y}_{c l-o p}\left(\omega^{L}, z+z_{0}\right) \mathbb{Y}_{c l-o p}\left(v ; z_{0}, \bar{z}_{0}\right) \mathbf{1}_{o p}\right)_{o p} \\
& =\left(B_{z_{0}}(u),\left(1-\frac{z}{\bar{z}_{0}-z_{0}}\right)^{-4}\left(\bar{z}_{0}-z_{0}\right)^{-2} \mathbb{Y}\left(\omega^{L}, f(z)\right) v_{1}\right)_{c l},
\end{aligned}
$$


where $f$ is the composition of the following maps:

$$
w \mapsto w+z_{0} \mapsto-\frac{\left(w+z_{0}\right)-z_{0}}{\left(w+z_{0}\right)-\bar{z}_{0}}=\frac{1}{\bar{z}_{0}-z_{0}} \frac{w}{1-\frac{w}{z_{0}-z_{0}}}
$$

which maps the domain $\mathbb{H}-z_{0}$ to the unit disk. Since $g_{1}\left(\omega^{L}, z\right)$ is analytic and free of singularities for $z+z_{0} \in \mathbb{H} \backslash\left\{z_{0}\right\}$, the right hand side of (2.41) can also be extended to an analytic function in $z \in \mathbb{H}-z_{0} \backslash\{0\}$. If we view $f(z)$ as a new variable $\xi$, then the right hand side of (2.41) can be extended to an analytic function on $\{\xi|1>| \xi \mid>0\}$, which has a Laurent series expansion. By the uniqueness of Laurent expansion, the right hand side of (2.41) gives exactly such a Laurent expansion and thus is absolutely convergent in $\{\xi|1>| \xi \mid>0\}$. Moreover, $\lim _{z+z_{0} \rightarrow r} g_{1}\left(\omega^{L}, z\right)$ exists for all $r \in \hat{\mathbb{R}}$. By the properties of Laurent series, the right hand side of (2.41) must converge absolutely for all $f(z) \in\{\xi|| \xi \mid=1\}$ to the function given by $\lim _{z+z_{0} \rightarrow r} g_{1}\left(\omega^{L}, z\right), r \in \hat{\mathbb{R}}$.

Similarly, for all $0<|\bar{z}|<\left|\operatorname{Im} z_{0}\right|$ and $\left|z+z_{0}\right|>\left|z_{0}\right|$, we have

$$
\begin{aligned}
& \left(u, \mathbb{Y}_{c l-o p}\left(\omega^{R}, \overline{z+z_{0}}\right) \mathbb{Y}_{c l-o p}\left(v ; z_{0}, \bar{z}_{0}\right) \mathbf{1}_{o p}\right)_{o p} \\
& =\left(B_{z_{0}}(u), e^{L(1)}\left(\bar{z}_{0}-z_{0}\right)^{-L(0)} \otimes e^{L(1)}{\overline{z_{0}}-z_{0}}^{-L(0)} \mathbb{Y}\left(\omega^{R}, \bar{z}\right) v\right)_{c l} \\
& =\left(B_{z_{0}}(u), \mathbb{Y}\left(e^{\left(1-\frac{\bar{z}}{z_{0}-\bar{z}_{0}}\right) L^{R}(1)}\left(1-\frac{z}{z_{0}-\bar{z}_{0}}\right)^{-2 L^{R}(0)}\left(z_{0}-\bar{z}_{0}\right)^{-L^{R}(0)} \omega^{R}, g(\bar{z})\right) v_{1}\right) c l \\
& =\left(1-\frac{\bar{z}}{z_{0}-\bar{z}_{0}}\right)^{-4}\left(z_{0}-\bar{z}_{0}\right)^{-2}\left(B_{z_{0}}(u), \mathbb{Y}\left(\omega^{R}, g(\bar{z})\right) v_{1}\right)_{c l},
\end{aligned}
$$

where $g$ is the composition of the following maps:

$$
w \mapsto w+\bar{z}_{0} \mapsto-\frac{\left(w+\bar{z}_{0}\right)-z_{0}}{\left(w+\bar{z}_{0}\right)-\bar{z}_{0}} \mapsto-\frac{\left(w+\bar{z}_{0}\right)-\bar{z}_{0}}{\left(w+\bar{z}_{0}\right)-z_{0}}=\frac{1}{z_{0}-\bar{z}_{0}} \frac{w}{1-\frac{w}{z_{0}-\bar{z}_{0}}}
$$

which maps the domain $-\mathbb{H}-\bar{z}_{0}$ to the unit disk. Moreover, the right hand side of (2.42), as a Laurent series of $g(\bar{z})$, is absolutely convergent for all $g(\bar{z}) \in\{\xi|| \xi \mid=1\}$ to $\lim _{z+z_{0} \rightarrow r} g_{2}\left(\omega^{R}, z\right), r \in \hat{\mathbb{R}}$.

Also notice that

$$
g\left(r-\bar{z}_{0}\right)=\frac{1}{f\left(r-z_{0}\right)} \in\left\{e^{i \theta} \mid 0 \leq \theta<2 \pi\right\}
$$

for all $r \in \hat{\mathbb{R}}$. Using (2.38) and by replacing $z$ in (2.41) by $r-z_{0}$ and $\bar{z}$ in (2.42) by $r-\bar{z}_{0}$, we obtain the following identity:

$$
\left(B_{z_{0}}(u), \mathbb{Y}\left(\omega^{L}, e^{i \theta}\right) v_{1}\right)_{c l}=\left(B_{z_{0}}(u), \mathbb{Y}\left(\omega^{R}, e^{-i \theta}\right) v_{1}\right)_{c l} e^{-4 i \theta},
$$

where $e^{i \theta}=f\left(r-z_{0}\right)$, for all $0 \leq \theta<2 \pi$. Notice that the existence of both sides of (2.44) follows directly from (2.38), which further follows from the condition of $u$ being vacuum-like. Then we obtain

$$
\sum_{n \in \mathbb{Z}}\left(B_{z_{0}}(u), L^{L}(n) v_{1}\right)_{c l} e^{i \theta(-n-2)}=\sum_{n \in \mathbb{Z}}\left(B_{z_{0}}(u), L^{R}(-n) v_{1}\right)_{c l} e^{i \theta(-n-2)}
$$

for all $0 \leq \theta<2 \pi$. Notice that $v_{1}$ can be arbitrary. Therefore, we must have (2.35) when $L(-1) u=0$. 
In physics, boundary states are usually obtained by solving Eq. (2.35). The solutions of such equation was first obtained by Ishibashi [I]. They are called Ishibashi states. The definition of boundary states we give in (2.34) is more general. Boundary conditions are also called "D-branes" in string theory. If $u$ is not a vacuum-like vector, the boundary state (2.34) associated with $u$ is also very interesting in physics (see for example [FFFS1]). Such boundary states are associated to the geometry on D-branes. In the end of Sect. 5.2, we will give a more natural (or algebraic) definition of D-brane.

\section{Cardy Condition}

In this section, we derive the Cardy condition from the axioms of open-closed partial conformal field theory by writing out the algebraic realizations of the both sides of Fig. 3 explicitly. Then we reformulate the Cardy condition in the framework of the intertwining operator algebra. Throughout this section, we fix an open-closed field algebra over $V$ given in (2.12) equipped with nondegenerate invariant bilinear forms $(\cdot, \cdot)_{o p}$ and $(\cdot, \cdot)_{c l}$.

3.1. The first version. In the Swiss-cheese dioperad, we exclude an interior sewing operation between two disks with strips and tubes and a self-sewing operation between two oppositely oriented boundary punctures on a single disk. The surface obtained after these two types of sewing operations can be the same cylinder or annulus. The axioms of open-closed partial conformal field theory require that the algebraic realization of these two sewing operations must coincide. This gives a nontrivial condition called Cardy condition (recall Fig. 3).

Although the Cardy condition only involves genus-zero surfaces, its algebraic realization is genus-one in nature. This fact is manifest if we consider the doubling map $\delta$. A double of a cylinder is actually a torus. Hence the Cardy condition is a condition on the equivalence of two algebraic realizations of two different decompositions of a torus. This is nothing but a condition associated to modularity.

That an annulus can be obtained by two different sewing operations is also shown in Fig. 4. In particular, the surface (A) in Fig. 4 shows how an annulus is obtained by sewing two oppositely oriented boundary punctures on the same disk with strips and tubes in $\mathbb{S}(1,3 \mid 0,0)$, and surface (C) in Fig. 4, viewed as a propagator of the close string, can be obtained by sewing an element in $\mathbb{S}(0,1 \mid 1,0)$ with an element in $\mathbb{S}(0,1 \mid 0,1)$ along the interior punctures. We only show in Figure 4 a simple case in which there are only two boundary punctures and no interior puncture. In general, the number of boundary punctures and interior punctures can be arbitrary. However, all general cases can be reduced to this simple case by applying associativities. Notice that the two boundary punctures in this simple case can not be reduced further by the associativities. We only focus on this case in this work.

The conformal map $f$ between the surface (A) and (B) and $g$ between the surface (C) and (B) in Fig. 4 are given by

$$
\begin{aligned}
& f(w)=\frac{1}{2 \pi i} \log w, \\
& g(w)=\frac{-\tau}{2 \pi i} \log w .
\end{aligned}
$$

It is also useful to know their inverses $f^{-1}(w)=e^{2 \pi i w}, g^{-1}(w)=e^{2 \pi i\left(-\frac{1}{\tau}\right) w}$. For any $z \in \mathbb{C}$, we set $q_{z}:=e^{2 \pi i z}$ and $p_{z}:=e^{2 \pi i\left(-\frac{1}{\tau}\right) z}$. The radius of the outer circle of the 

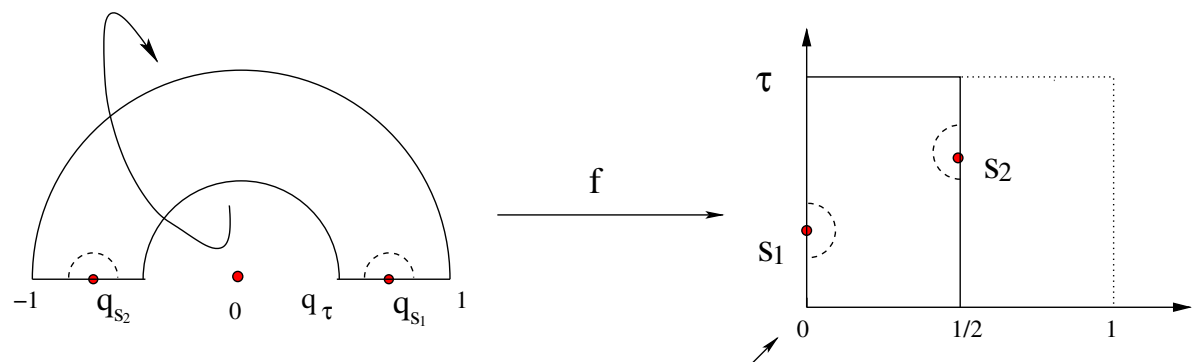

(A)

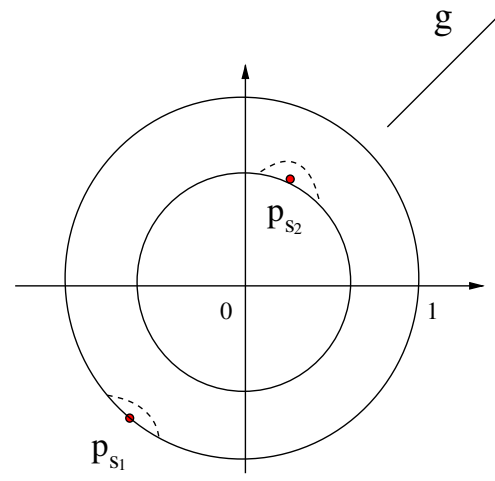

(B)

(C)

Fig. 4. Cardy condition: two different sewings give same annulus

surface $(C)$ in Fig. 4 is $\left|p_{s_{1}}\right|=1$ and that of the inner circle is

$$
\left|p_{s_{2}}\right|=e^{\pi i\left(-\frac{1}{\tau}\right)}=q_{-\frac{1}{\tau}}^{1 / 2} .
$$

As we have argued and will show more explicitly later, the Cardy condition is deeply related to modularity. In [H9], Huang introduced the so-called geometrically modified intertwining operators, which is very convenient for the study of modularity. He was motivated by the fact that it is much easier to study modularity in the global coordinates. Namely, one should choose the local coordinates at $s_{1}, s_{2}$ in surface (B) in Fig. 4 as simple as possible. More precisely, we choose the local coordinates at $s_{1}, s_{2}$ as

$$
\begin{aligned}
& w \mapsto e^{\frac{\pi i}{2}}\left(w-s_{1}\right), \\
& w \mapsto e^{-\frac{\pi i}{2}}\left(w-s_{2}\right)
\end{aligned}
$$

respectively. Correspondingly, the local coordinates at punctures $q_{s_{1}}, q_{s_{2}}$ are

$$
\begin{aligned}
& f_{q_{s_{1}}}(w)=\left.e^{\frac{\pi i}{2}} \frac{1}{2 \pi i} \log \left(1+\frac{1}{q_{s_{1}}} x\right)\right|_{x=w-q_{s_{1}}}, \\
& f_{q_{s_{2}}}(w)=\left.e^{-\frac{\pi i}{2}} \frac{1}{2 \pi i} \log \left(1+\frac{1}{q_{s_{2}}} x\right)\right|_{x=w-q_{s_{2}}}
\end{aligned}
$$

respectively. 
Notice that both local coordinates $f_{q_{s_{1}}}(w)$ and $f_{q_{s_{2}}}(w)$ are real analytic. Hence $\exists B_{j}^{(i)} \in \mathbb{R}, b_{0}^{(i)} \in \mathbb{R}_{+}, i=1,2$ such that

$$
\begin{aligned}
& f_{q_{s_{1}}}(w)=\left.e^{\sum_{j=1}^{\infty} B_{j}^{(1)} x^{j+1} \frac{d}{d x}}\left(b_{0}^{(1)}\right)^{x \frac{d}{d x}} x\right|_{x=w-q_{s_{1}}}, \\
& f_{q_{s_{2}}}(w)=\left.e^{\sum_{j=1}^{\infty} B_{j}^{(2)} x^{j+1} \frac{d}{d x}}\left(b_{0}^{(2)}\right)^{x \frac{d}{d x}} x\right|_{x=w-q_{s_{2}}} .
\end{aligned}
$$

Then the algebraic realization of the surface (A) gives a map $V_{o p} \otimes V_{o p} \rightarrow \mathbb{C}$. We assume $1>\left|q_{s_{1}}\right|>\left|q_{s_{2}}\right|>\left|q_{\tau}\right|>0$. By the axiom of open-closed conformal field theory, this map must be given by (recall (1.25))

$$
v_{1} \otimes v_{2} \mapsto \operatorname{Tr}_{V_{o p}}\left(Y_{o p}\left(T_{q_{s_{1}}} v_{1}, q_{s_{1}}\right) Y_{o p}\left(T_{q_{s_{2}}} v_{2}, q_{s_{2}}\right) q_{\tau}^{L(0)}\right),
$$

where

$$
\begin{aligned}
& T_{q_{s_{1}}}=e^{-\sum_{j=1}^{\infty} B_{j}^{(1)} L(j)}\left(b_{0}^{(1)}\right)^{-L(0)}, \\
& T_{q_{s_{2}}}=e^{-\sum_{j=1}^{\infty} B_{j}^{(2)} L(j)}\left(b_{0}^{(2)}\right)^{-L(0)} .
\end{aligned}
$$
by

We need to rewrite $T_{q_{s_{1}}}$ and $T_{q_{s_{2}}}$. Let $A_{j}, j \in \mathbb{Z}_{+}$, be the complex numbers defined

$$
\log (1+y)=e^{\sum_{j=1}^{\infty} A_{j} y^{j+1} \frac{d}{d y}} y .
$$

It is clear that $A_{j} \in \mathbb{R}$. Hence we also obtain:

$$
\frac{1}{2 \pi i} \log \left(1+\frac{1}{z} x\right)=z^{-x \frac{d}{d x}} e^{\sum_{j \in \mathbb{Z}_{+}} A_{j} x^{j+1} \frac{d}{d x}}(2 \pi i)^{-x \frac{d}{d x}} x .
$$

\section{Lemma 3.1.}

$$
\begin{aligned}
& T_{q_{s_{1}}}=\left(q_{s_{1}}\right)^{L(0)} e^{-\sum_{j=1}^{\infty} A_{j} L(j)}(2 \pi i)^{L(0)} e^{-\frac{\pi i}{2} L(0)}, \\
& T_{q_{s_{2}}}=\left(q_{s_{2}}\right)^{L(0)} e^{-\sum_{j=1}^{\infty} A_{j} L(j)}(2 \pi i)^{L(0)} e^{-2 \pi i L(0)} e^{\frac{\pi i}{2} L(0)} .
\end{aligned}
$$

Proof. By results in [BHL], if $e^{\sum_{j=1}^{\infty} C_{j} L(j)} c_{0}^{L(0)}=e^{\sum_{j=1}^{\infty} D_{j} L(j)} d_{0}^{L(0)}$ for any $C_{j}$, $D_{j} \in \mathbb{C}, c_{0}, d_{0} \in \mathbb{C}$, we must have $C_{j}=D_{j}$ and $c_{0}=d_{0}$. Therefore, by moving the factor $\left(q_{S_{1}}\right)^{L(0)}$ to the right side of $e^{-\sum_{j=1}^{\infty} A_{j} L(j)}$ in (3.9) and similarly moving the factor $\left(q_{s_{2}}\right)^{L(0)}$ in (3.9), we see that it is enough to show

$$
\begin{aligned}
& \left(b_{0}^{(1)}\right)^{L(0)}=\left(q_{s_{1}}\right)^{L(0)}(2 \pi i)^{L(0)} e^{-\frac{\pi i}{2} L(0)}, \\
& \left(b_{0}^{(1)}\right)^{L(0)}=\left(q_{s_{2}}\right)^{L(0)}(2 \pi i)^{L(0)} e^{-2 \pi i L(0)} e^{\frac{\pi i}{2} L(0)} .
\end{aligned}
$$

Using our conventions $(0.1)(0.2)$, it is easy to check that the above identities hold. 
Let $W$ be a $V$-module. Huang introduced the following operator in [H9]:

$$
\mathcal{U}(x):=x^{L(0)} e^{-\sum_{j=1}^{\infty} A_{j} L(j)}(2 \pi i)^{L(0)} \in(\text { End } W)\{x\} .
$$

Thus (3.6) can be rewritten as follows:

$$
v_{1} \otimes v_{2} \mapsto \operatorname{Tr}_{V_{o p}}\left(Y_{o p}\left(\mathcal{U}\left(q_{s_{1}}\right) v_{1}^{\prime}, q_{s_{1}}\right) Y_{o p}\left(\mathcal{U}\left(q_{s_{2}}\right) e^{-2 \pi i L(0)} v_{2}^{\prime}, q_{s_{2}}\right) q_{\tau}^{L(0)}\right),
$$

where $v_{1}^{\prime}=e^{-\frac{\pi i}{2} L(0)} v_{1}$ and $v_{2}^{\prime}=e^{\frac{\pi i}{2} L(0)} v_{2}$.

Now we consider the algebraic realization of the surface (C) in Fig. 4 obtained from an interior sewing operation between an element in $\mathbb{S}(0,1 \mid 1,0)$ and an element in $\mathbb{S}(0,1 \mid 0,1)$.

Lemma 3.2. $\forall r \in(0,1) \subset \mathbb{R}_{+}$, the surface $C$ in Fig. 4 is conformally equivalent to a surface $Q_{1}{ }_{1} \infty_{1}^{I} Q_{2}$, where

1. $Q_{1} \in \mathbb{S}(0,1 \mid 0,1)$ with punctures at $z_{1} \in \mathbb{H}, \infty \in \hat{\mathbb{R}}$ and local coordinates:

$$
\begin{aligned}
& f_{z_{1}}: w \mapsto\left(\frac{p_{s_{1}}}{r}\right) \frac{w-z_{1}}{w-\bar{z}_{1}}, \\
& f_{\infty}^{(1)}: w \mapsto e^{\frac{\pi i}{2}}\left(\frac{-\tau}{2 \pi i}\right) \log \frac{w-z_{1}}{w-\bar{z}_{1}} ;
\end{aligned}
$$

2. $Q_{2} \in \mathbb{S}(0,1 \mid 1,0)$ with punctures at $z_{2} \in \mathbb{H}, \infty \in \hat{\mathbb{R}}$ with local coordinates

$$
\begin{aligned}
f_{z_{2}}: w & \mapsto\left(\frac{-r}{p_{s_{2}}}\right) \frac{w-z_{2}}{w-\bar{z}_{2}}, \\
f_{\infty}^{(2)}: w & \mapsto e^{-\frac{\pi i}{2}}\left(\frac{-\tau}{2 \pi i}\right) \log \frac{w-\bar{z}_{2}}{w-z_{2}} .
\end{aligned}
$$

Proof. Let us first define two disks $D_{1}$ and $D_{2} . D_{1}$ is the unit disk, i.e. $D_{1}:=\{z \in$ $\mathbb{C}|| z \mid \leq 1\}$, which has punctures at $p_{s_{1}}, 0$ and local coordinates:

$$
\begin{aligned}
g_{p_{s_{1}}}: w & \mapsto e^{\frac{\pi i}{2}}\left(\frac{-\tau}{2 \pi i} \log w-s_{1}\right), \\
g_{0}: w & \mapsto r^{-1} w .
\end{aligned}
$$

$D_{2}$ is the disk $\left\{z \in \hat{\mathbb{C}}|| z|\geq| p_{s_{2}} \mid\right\}$ which has punctures at $p_{s_{2}}, \infty$ and local coordinates:

$$
\begin{aligned}
g_{p_{s_{2}}}: w & \mapsto e^{-\frac{\pi i}{2}}\left(\frac{-\tau}{2 \pi i} \log w-s_{2}\right), \\
g_{\infty}: w & \mapsto \frac{-r}{w} .
\end{aligned}
$$

It is not hard to see that the surface $C$ in Fig. 1 can be obtained by sewing the puncture $0 \in D_{1}$ with the puncture $\infty \in D_{2}$ according to the usual definition of the interior sewing operation. Then it is enough to show that $D_{1}$ and $D_{2}$ are conformally equivalent 
to $P$ and $Q$ respectively. We define two maps $h_{1}: Q_{1} \rightarrow D_{1}$ and $h_{2}: Q_{2} \rightarrow D_{2}$ as follows:

$$
\begin{aligned}
& h_{1}: w \mapsto p_{s_{1}} \frac{w-z_{1}}{w-\bar{z}_{1}}, \\
& h_{2}: w \mapsto p_{s_{2}} \frac{w-\bar{z}_{2}}{w-z_{2}} .
\end{aligned}
$$

It is clear that $h_{1}$ and $h_{2}$ are both biholomorphic. We can check directly that $h_{1}$ and $h_{2}$ map punctures to punctures and preserve local coordinates as well.

Using (2.33), we obtain the algebraic realization of the annulus $C$ in Fig. 1 as follows: $v_{1} \otimes v_{2} \mapsto\left(\left(T_{1}^{L} \otimes T_{1}^{R}\right)^{*} \iota_{c l-o p}^{*}\left(z_{1}, \bar{z}_{1}\right)\left(T_{2} v_{1}^{\prime}\right),\left(T_{3}^{L} \otimes T_{3}^{R}\right)^{*} \iota_{c l-o p}^{*}\left(z_{2}, \bar{z}_{2}\right)\left(T_{4} v_{2}^{\prime}\right)\right)_{c l}$,

where $T_{1}^{L, R}, T_{2}, T_{3}^{L, R}, T_{4}$ are conformal transformations determined by local coordinates $f_{z_{1}}, f_{\infty}^{(2)}, f_{z_{2}}^{(1)}, f_{\infty}^{(2)}$, and $\left(T_{i}^{L} \otimes T_{i}^{R}\right)^{*}$ is the adjoint of $T_{i}^{L} \otimes T_{i}^{R}$ with respect to the bilinear form $(\cdot, \cdot)_{c l}$ for $i=1,3$, and $v_{1}^{\prime}=e^{-\frac{\pi i}{2} L(0)} v_{1}$ and $v_{2}^{\prime}=e^{\frac{\pi i}{2} L(0)} v_{2}$.

Lemma 3.3. Recall the convention (0.2), we have

$$
\begin{aligned}
T_{1}^{L} & =\left(z_{1}-\bar{z}_{1}\right)^{L(0)} e^{L(1)}\left(\frac{p_{s_{1}}}{r}\right)^{-L(0)}, T_{1}^{R}={\overline{\left(z_{1}-\bar{z}_{1}\right)}}^{L(0)} e^{L(1)}{\overline{\left(\frac{p_{s_{1}}}{r}\right)}}^{-L(0)}, \\
T_{2} & =e^{\bar{z}_{1} L(1)}\left(z_{1}-\bar{z}_{1}\right)^{-L(0)} \mathcal{U}(1)\left(-\frac{1}{\tau}\right)^{L(0)}, \\
T_{3}^{L} & =\left(\bar{z}_{2}-z_{2}\right)^{L(0)} e^{-L(1)}\left(\frac{p_{s_{2}}}{r}\right)^{L(0)}, T_{3}^{R}={\overline{\left(\bar{z}_{2}-z_{2}\right)}}^{L(0)} e^{-L(1)}{\overline{\left(\frac{p_{s_{2}}}{r}\right)}}^{L(0)}, \\
T_{4} & =e^{z_{2} L(1)}\left(\bar{z}_{2}-z_{2}\right)^{-L(0)} \mathcal{U}(1)\left(-\frac{1}{\tau}\right)^{L(0)} .
\end{aligned}
$$

Proof. From (3.12) and (3.14), we obtain

$$
\begin{aligned}
& f_{z_{1}}:\left.w \mapsto\left(z_{1}-\bar{z}_{1}\right)^{-x \frac{d}{d x}} e^{-x^{2} \frac{d}{d x}}\left(\frac{p_{s_{1}}}{r}\right)^{x \frac{d}{d x}} x\right|_{x=w-z_{1}}, \\
& f_{z_{2}}:\left.w \mapsto\left(\bar{z}_{2}-z_{2}\right)^{-x \frac{d}{d x}} e^{x^{2} \frac{d}{d x}}\left(\frac{p_{s_{2}}}{r}\right)^{-x \frac{d}{d x}} x\right|_{x=w-z_{2}} .
\end{aligned}
$$

Then (3.21) and (3.23) is obvious. Notice that the expression (3.21) is independent of our choice of branch cut as long as we keep the convention (0.2).

From (3.13) and (3.15), we obtain

$$
\begin{aligned}
& f_{\infty}^{(1)}(w)=\left.e^{-\bar{z}_{1} x^{2} \frac{d}{d x}}\left(z_{1}-\bar{z}_{1}\right)^{x \frac{d}{d x}} e^{\sum_{j=1}^{\infty} A_{j} x^{j+1} \frac{d}{d x}}(2 \pi i)^{-x \frac{d}{d x}}\left(-\frac{1}{\tau}\right)^{-x \frac{d}{d x}} e^{\frac{\pi i}{2} x \frac{d}{d x} x}\right|_{x=\frac{-1}{w}}, \\
& f_{\infty}^{(2)}(w)=\left.e^{-z_{2} x^{2} \frac{d}{d x}}\left(\bar{z}_{2}-z_{2}\right)^{x \frac{d}{d x}} e^{\sum_{j=1}^{\infty} A_{j} x^{j+1} \frac{d}{d x}}(2 \pi i)^{-x \frac{d}{d x}}\left(-\frac{1}{\tau}\right)^{-x \frac{d}{d x}} e^{-\frac{\pi i}{2} x \frac{d}{d x}} x\right|_{x=\frac{-1}{w}} .
\end{aligned}
$$


Recall that $f_{\infty}^{(1)}, f_{\infty}^{(2)}$ are both real analytic. Similar to the proof of Lemma 3.1, to show (3.22) and (3.24) it is enough to show that

$$
\begin{aligned}
& \left(b_{\infty}^{(1)}\right)^{L(0)}=\left(z_{1}-\bar{z}_{1}\right)^{-L(0)}(2 \pi i)^{L(0)}\left(-\frac{1}{\tau}\right)^{L(0)} e^{-\frac{\pi i}{2} L(0)} \\
& \left(b_{\infty}^{(2)}\right)^{L(0)}=\left(\bar{z}_{2}-z_{2}\right)^{-L(0)}(2 \pi i)^{L(0)}\left(-\frac{1}{\tau}\right)^{L(0)} e^{\frac{\pi i}{2} L(0)}
\end{aligned}
$$

for some $b_{\infty}^{(1)}, b_{\infty}^{(2)} \in \mathbb{R}_{+}$. Using our convention (0.1) and (0.2), it is a direct check that (3.28) and (3.29) holds.

Combining (3.11), (3.20) and additional natural factors $q_{\tau}^{-\frac{c}{24}}, q_{-\frac{1}{\tau}}^{-\frac{c}{24}}$ (see $[\mathrm{Z}, \mathrm{H} 9]$ ), which is due to the determinant line bundle on torus [Se1, Kr], we obtain the following formulation of the Cardy condition:

Definition 3.4. The open-closed field algebra over $V$ given in (2.12) and equipped with nondegenerate bilinear forms $(\cdot, \cdot)_{o p}$ and $(\cdot, \cdot)_{c l}$ is said to satisfy Cardy condition if the left hand sides of the following formula, $\forall z_{1}, z_{2} \in \mathbb{H}, v_{1}, v_{2} \in V_{o p}$,

$$
\begin{aligned}
& \operatorname{Tr}_{V_{o p}}\left(Y_{o p}\left(\mathcal{U}\left(q_{s_{1}}\right) v_{1}, q_{s_{1}}\right) Y_{o p}\left(\mathcal{U}\left(q_{s_{2}}\right) e^{-2 \pi i L(0)} v_{2}, q_{s_{2}}\right) q_{\tau}^{L(0)-c / 24}\right) \\
& \quad=\left(\left(T_{1}^{L} \otimes T_{1}^{R}\right)^{*} \iota_{c l-o p}^{*}\left(z_{1}, \bar{z}_{1}\right)\left(T_{2} v_{1}\right), q_{-\frac{1}{\tau}}^{-c / 24}\left(T_{3}^{L} \otimes T_{3}^{R}\right)^{*} \iota_{c l-o p}^{*}\left(z_{2}, \bar{z}_{2}\right)\left(T_{4} v_{2}\right)\right)_{c l}
\end{aligned}
$$

converge absolutely when $1>\left|q_{s_{1}}\right|>\left|q_{s_{2}}\right|>\left|q_{\tau}\right|>0$, and the right hand side of (3.30) converge absolutely for all $s_{1}, s_{2} \in \mathbb{H}$ satisfying $\operatorname{Re} s_{1}=0, \operatorname{Re} s_{2}=\frac{1}{2}$. Moreover, Eq. (3.30) holds when $1>\left|q_{s_{1}}\right|>\left|q_{s_{2}}\right|>\left|q_{\tau}\right|>0$.

Remark 3.5. The dependence of $z_{1}, z_{2}, r$ of the right hand side of (3.30) is superficial as required by the independence of $z_{1}, z_{2}, r$ of the left hand side of (3.30). We will see it more explicitly later.

Using the definition of boundary states (2.34), (3.30) can also be written as follows:

$$
\begin{aligned}
& \operatorname{Tr}_{V_{o p}}\left(Y_{o p}\left(\mathcal{U}\left(q_{s_{1}}\right) v_{1}, q_{s_{1}}\right) Y_{o p}\left(\mathcal{U}\left(q_{s_{2}}\right) e^{-2 \pi i L(0)} v_{2}, q_{s_{2}}\right) q_{\tau}^{L(0)-c / 24}\right) \\
& =\left(B_{z_{1}}\left(T_{2} v_{1}\right),\left(\frac{p_{s_{2}}}{-p_{s_{1}}}\right)^{L(0)} \otimes\left(\overline{\frac{p_{s_{2}}}{-p_{s_{1}}}}\right)^{L(0)} q_{-\frac{1}{\tau}}^{-c / 24} B_{z_{2}}\left(T_{4} v_{2}\right)\right)_{c l}
\end{aligned}
$$

3.2. The second version. In this subsection, we rewrite the Cardy condition (3.30) in the framework of the intertwining operator algebra.

Since $V$ satisfies the conditions in Theorem 0.1 , it has only finite number of inequivalent irreducible modules. Let $\mathcal{I}$ be the set of equivalence classes of irreducible $V$-modules. We denote the equivalence class of the adjoint module $V$ as $e$, i.e. $e \in \mathcal{I}$. Let $W_{a}$ be a chosen representative of $a \in \mathcal{I}$. 
For any $V$-module $\left(W, Y_{W}\right)$, we denote the graded dual space of $W$ as $W^{\prime}$, i.e. $W^{\prime}=\oplus_{n \in \mathbb{C}}\left(W_{(n)}\right)^{*}$. There is a contragredient module structure on $W^{\prime}$ [FHL] given by a vertex operator $Y_{W}^{\prime}$, which is defined as follows:

$$
\left\langle Y_{W}^{\prime}(u, x) w^{\prime}, w\right\rangle:=\left\langle w^{\prime}, Y_{W}\left(e^{-x L(1)} x^{-2 L(0)} u,-x^{-1}\right) w\right\rangle
$$

for $u \in V, w \in W, w^{\prime} \in W^{\prime}$. ( $\left.W^{\prime}, Y_{W}^{\prime}\right)$ (or simply $W$ ) is the only module structure on $W^{\prime}$ we use in this work. So we can set $Y_{W^{\prime}}:=Y_{W}^{\prime}$. We denote the equivalent class of $W_{a}^{\prime}$ as $a^{\prime}$. It is harmless to set $W_{a}^{\prime}=W_{a^{\prime}}$. Moreover, $W^{\prime \prime}$ is canonically identified with $W$. Hence $a^{\prime \prime}=a$ for $a \in \mathcal{I}$.

By assumption on $V, V^{\prime} \cong V$, i.e. $e^{\prime}=e$. From [FHL], there is a nondegenerate invariant bilinear form $(\cdot, \cdot)$ on $V$ such that $(\mathbf{1}, \mathbf{1})=1$. This bilinear form specifies a unique isomorphism from $V$ to $V^{\prime}$. In the rest of this work, we identify $V^{\prime}$ with $V$ using this isomorphism without mentioning it explicitly.

For any triple $V$-modules $W_{1}, W_{2}, W_{3}$, we have isomorphisms

$$
\Omega_{r}: \mathcal{V}_{W_{1} W_{2}}^{W_{3}} \rightarrow \mathcal{V}_{W_{2} W_{1}}^{W_{3}}, \quad \forall r \in \mathbb{Z}
$$

given as follows:

$$
\Omega_{r}(\mathcal{Y})\left(w_{2}, z\right) w_{1}=e^{z L(-1)} \mathcal{Y}\left(w_{1}, e^{(2 r+1) \pi i} z\right) w_{2},
$$

for $\mathcal{Y} \in \mathcal{V}_{W_{1} W_{2}}^{W_{3}}$ and $w_{i} \in W_{i}, i=1,2$. The following identity

$$
\Omega_{r} \circ \Omega_{-r-1}=\Omega_{-r-1} \circ \Omega_{r}=\mathrm{id}
$$

is proved in [HL3].

For $\mathcal{Y} \in \mathcal{V}_{W_{1} W_{2}}^{W_{3}}$ and $r \in \mathbb{Z}$, a so-called $r$-contragredient operator $A_{r}(\mathcal{Y})$ was introduced in [HL3]. Here, we use two slightly different operators $\tilde{A}_{r}(\mathcal{Y})$ and $\hat{A}_{r}(\mathcal{Y})$ introduced in [Ko1] and defined as follows:

$$
\begin{aligned}
& \left\langle\tilde{A}_{r}(\mathcal{Y})\left(w_{1}, e^{(2 r+1) \pi i} x\right) w_{3}^{\prime}, w_{2}\right\rangle=\left\langle w_{3}^{\prime}, \mathcal{Y}\left(e^{x L(1)} x^{-2 L(0)} w_{1}, x^{-1}\right) w_{2}\right\rangle, \\
& \left\langle\hat{A}_{r}(\mathcal{Y})\left(w_{1}, x\right) w_{3}^{\prime}, w_{2}\right\rangle=\left\langle w_{3}^{\prime}, \mathcal{Y}\left(e^{-x L(1)} x^{-2 L(0)} w_{1}, e^{(2 r+1) \pi i} x^{-1}\right) w_{2}\right\rangle,
\end{aligned}
$$

for $\mathcal{Y} \in \mathcal{V}_{W_{1} W_{2}}^{W_{3}}$ and $w_{1} \in W_{1}, w_{2} \in W_{2}, w_{3}^{\prime} \in W_{3}^{\prime}$. In particular, when $W_{1}=V$ and $W_{2}=W_{3}=W$, we have $\tilde{A}_{r}\left(Y_{W}\right)=\hat{A}_{r}\left(Y_{W}\right)=Y_{W}^{\prime}=Y_{W^{\prime}}, \forall r \in \mathbb{Z}$. If $\mathcal{Y} \in \mathcal{V}_{W_{1} W_{2}}^{W_{3}}$, then $\tilde{A}_{r}(\mathcal{Y}), \hat{A}_{r}(\mathcal{Y}) \in \mathcal{V}_{W_{1} W_{3}^{\prime}}^{W^{\prime}}$ for $r \in \mathbb{Z}$ and

$$
\tilde{A}_{r} \circ \hat{A}_{r}(\mathcal{Y})=\hat{A}_{r} \circ \tilde{A}_{r}(\mathcal{Y})=\mathcal{Y} .
$$

Let $\mathcal{Y} \in \mathcal{V}_{a_{1} a_{2}}^{a_{3}}$. We define $\sigma_{123}:=\Omega_{r} \circ \tilde{A}_{r}$. It is easy to see that

$$
\begin{aligned}
& \left\langle w_{a_{3}}^{\prime}, \mathcal{Y}\left(w_{a_{1}}, x\right) w_{a_{2}}\right\rangle \\
& \quad=\left\langle e^{-x^{-1} L(-1)} \sigma_{123}(\mathcal{Y})\left(w_{a_{3}}^{\prime}, x^{-1}\right) e^{-x L(1)} x^{-2 L(0)} w_{a_{1}}, w_{a_{2}}\right\rangle
\end{aligned}
$$

for $w_{a_{1}} \in W_{a_{1}}, w_{a_{2}} \in W_{a_{2}}, w_{a_{3}}^{\prime} \in W_{a_{3}}^{\prime}$. It is also clear that $\sigma_{123}$ is independent of $r \in \mathbb{Z}$. It is proved in [Ko1] that $\sigma_{123}^{3}=\mathrm{id}_{\mathcal{V}_{a_{1} a_{2}}^{a_{3}}}$. We also denote $\sigma_{123}^{-1}$ as $\sigma_{132}$. Clearly, we have $\sigma_{132}=\hat{A}_{r} \circ \Omega_{-r-1}$ and

$$
\begin{aligned}
& \left\langle\sigma_{132}(\mathcal{Y})\left(w_{1}, x\right) w_{3}^{\prime}, w_{2}\right\rangle \\
& =\left\langle w_{3}^{\prime}, e^{-x^{-1} L(-1)} \mathcal{Y}\left(w_{2}, x^{-1}\right) e^{-x L(1)} x^{-2 L(0)} w_{1}\right\rangle
\end{aligned}
$$

for $w_{a_{1}} \in W_{a_{1}}, w_{a_{2}} \in W_{a_{2}}, w_{a_{3}}^{\prime} \in W_{a_{3}}^{\prime}$. 
For any $V$-module $W$, we define a $V$-module map $\theta_{W}: W \rightarrow W$ by

$$
\theta_{W}: w \mapsto e^{-2 \pi i L(0)} w
$$

For $W_{a}$, we have $\theta_{W_{a}}=e^{-2 \pi i h_{a}} \mathrm{id}_{W_{a}}$, where $h_{a} \in \mathbb{C}$ is the lowest conformal weight of $W_{a}$.

We denote the graded dual space of $V_{c l}$ and $V_{o p}$ by $V_{c l}^{\prime}$ and $V_{o p}^{\prime}$ respectively. Let $\varphi_{c l}: V_{c l} \rightarrow V_{c l}^{\prime}$ and $\varphi_{o p}: V_{o p} \rightarrow V_{o p}^{\prime}$ be the isomorphisms induced from $(\cdot, \cdot)_{c l}$ and $(\cdot, \cdot)_{o p}$ respectively. Namely, we have

$$
\begin{aligned}
& \left(u_{1}, u_{2}\right)_{c l}=\left\langle\varphi_{c l}\left(u_{1}\right), u_{2}\right\rangle \\
& \left(v_{1}, v_{2}\right)_{o p}=\left\langle\varphi_{o p}\left(v_{1}\right), v_{2}\right\rangle
\end{aligned}
$$

for $u_{1}, u_{2} \in V_{c l}$ and $v_{1}, v_{2} \in V_{o p}$.

$V_{c l}$ as a conformal full field algebra over $V \otimes V$ can be expanded as follows:

$$
V_{c l}=\oplus_{i=1}^{N_{c l}} W_{r_{L}(i)} \otimes W_{r_{R}(i)}
$$

where $r_{L}, r_{R}:\left\{1, \ldots, N_{c l}\right\} \rightarrow \mathcal{I}$. For $a \in \mathcal{I}$, we choose a basis $\left\{e_{a ; \alpha}\right\}_{\alpha \in \mathbb{N}}$ of $W_{a}$ and a dual basis $\left\{e_{a ; \alpha}^{\prime}\right\}_{\alpha \in \mathbb{N}}$ of $W_{a}^{\prime}$. Then

$$
\left\{e_{r_{L}(i), \alpha} \otimes e_{r_{R}(i), \beta}\right\}_{i=1, \ldots, N_{c l}, \alpha, \beta \in \mathbb{N}}
$$

is a basis of $V_{c l}$ and

$$
\left\{\varphi_{c l}^{-1}\left(e_{r_{L}(i) ; \alpha}^{\prime} \otimes e_{r_{R}(i), \beta}^{\prime}\right)\right\}_{i=1, \ldots, N_{c l}, \alpha, \beta \in \mathbb{N}}
$$

is its dual basis with respect to the nondegenerate bilinear form $(\cdot, \cdot)_{c l}$.

Let $T: \mathcal{C}_{V \otimes V} \rightarrow \mathcal{C}_{V}$ be the tensor bifunctor. We showed in [Ko2] that there is a morphism $\iota_{c l-o p}: T\left(V_{c l}\right) \rightarrow V_{o p}$ in $\mathcal{C}_{V}$ (see (3.81),(3.82) in [Ko2] for the definition). We define a morphism $\iota_{c l-o p}^{\prime}: T\left(V_{c l}^{\prime}\right) \rightarrow V_{o p}$ as a composition of maps as follows:

$$
\iota_{c l-o p}^{\prime}: T\left(V_{c l}^{\prime}\right) \stackrel{T\left(\varphi_{c l}^{-1}\right)}{\longrightarrow} T\left(V_{c l}\right) \stackrel{\iota_{c l-o p}}{\longrightarrow} V_{o p}
$$

By the universal property of the tensor product $\otimes[\mathrm{HL} 1-\mathrm{HL} 4], \mathcal{V}_{W_{1} W_{2}}^{W_{3}}$ and $\operatorname{Hom}_{V}\left(W_{1} \otimes\right.$ $\left.W_{2}, W_{3}\right)$ for any three $V$-modules $W_{1}, W_{2}, W_{3}$ are canonically isomorphic. Given a morphism $m \in \operatorname{Hom}_{V}\left(W_{1} \otimes W_{2}, W_{3}\right)$, we denote the corresponding intertwining operator as $\mathcal{Y}_{m}$. Conversely, given an intertwining operator $\mathcal{Y}$, we denote its corresponding morphism as $m_{\mathcal{Y}}$. Therefore, we have two intertwining operators $\mathcal{Y}_{l_{c l-o p}}$ and $\mathcal{Y}_{l_{c l-o p}^{\prime}}$ corresponding to morphisms $\iota_{c l-o p}$ and $\iota_{c l-o p}^{\prime}$ respectively.

Lemma 3.6. For $z \in \mathbb{H}$, we have

$$
\iota_{c l-o p}(z, \bar{z})\left(e_{r_{L}(i), \alpha} \otimes e_{r_{R}(i), \beta}\right)=e^{\bar{z} L(-1)} \mathcal{Y}_{\iota_{c l-o p}}\left(e_{r_{L}(i), \alpha}, z-\bar{z}\right) e_{r_{R}(i), \alpha}
$$


Proof. It is proved in [Ko2] that

$$
m_{\mathbb{Y}_{c l-o p}}=m_{Y_{o p}^{f}} \circ\left(\iota_{c l-o p} \otimes \mathrm{id}_{V_{o p}}\right) .
$$

Using (3.46), when $z \in \mathbb{H}, \zeta \in \overline{\mathbb{H}}$ and $|\zeta|>|z-\zeta|>0$, we have

$$
\begin{aligned}
\iota_{c l-o p}(z, \zeta)\left(e_{r_{L}(i), \alpha} \otimes e_{r_{R}(i), \beta}\right) & =\mathbb{Y}_{c l-o p}\left(e_{r_{L}(i), \alpha} \otimes e_{r_{R}(i), \beta} ; z, \zeta\right) \mathbf{1}_{o p} \\
& =Y_{o p}^{f}\left(\mathcal{Y}_{l_{c l-o p}}\left(e_{r_{L}(i), \alpha}, z-\zeta\right) e_{r_{R}}(i), \beta, \zeta\right) \mathbf{1} \\
& =e^{\zeta L(-1)} \mathcal{Y}_{l_{c l-o p}}\left(e_{r_{L}(i), \alpha}, z-\zeta\right) e_{r_{R}(i), \beta} .
\end{aligned}
$$

By the convergence property of the iterate of two intertwining operators, the right hand side of (3.47) is a power series of $\zeta$ absolutely convergent for $|\zeta|>|z-\zeta|>0$. By the property of power series, the right hand side of (3.47) must converge absolutely for all $z \in \mathbb{H}, \zeta \in \overline{\mathbb{H}}$. Because analytic extension in a simply connected domain is unique, we obtain that the equality (3.47) holds for all $z \in \mathbb{H}, \zeta \in \overline{\mathbb{H}}$. When $\zeta=\bar{z}$, we obtain (3.45).

Now we consider both sides of the Cardy condition (3.30) for an open-closed field algebra over $V$. On the left hand side of (3.30), we have $q_{s_{2}}<0$. Using (1.25) and (3.33), we obtain, $\forall v_{3} \in V_{o p}$,

$$
\begin{aligned}
Y_{o p}\left(\mathcal{U}\left(q_{s_{2}}\right) v_{2}, q_{s_{2}}\right) v_{3} & =e^{-\left|q_{s_{2}}\right| L(-1)} Y_{o p}\left(v_{3},\left|q_{s_{2}}\right|\right) \mathcal{U}\left(q_{s_{2}}\right) v_{2} \\
& =\Omega_{-1}\left(Y_{o p}^{f}\right)\left(\mathcal{U}\left(q_{s_{2}}\right) v_{2}, e^{\pi i}\left|q_{s_{2}}\right|\right) v_{3} .
\end{aligned}
$$

Hence we can rewrite the left hand side of (3.30) as follows:

$$
\operatorname{Tr}_{V_{o p}}\left(Y_{o p}^{f}\left(\mathcal{U}\left(q_{s_{1}}\right) v_{1}, q_{s_{1}}\right) \Omega_{-1}\left(Y_{o p}^{f}\right)\left(\mathcal{U}\left(q_{s_{2}}\right) e^{-2 \pi i L(0)} v_{2}, e^{\pi i}\left|q_{s_{2}}\right|\right) q_{\tau}^{L(0)-c / 24}\right)
$$

for $q_{s_{1}}>\left|q_{s_{2}}\right|>\left|q_{\tau}\right|>0$.

We have the following result for the right hand side of (3.30).

Proposition 3.7. For $s_{1}, s_{2} \in \mathbb{H}, \operatorname{Re} s_{1}=0, \operatorname{Re} s_{2}=0$,

$$
\begin{array}{r}
\left(\left(T_{1}^{L} \otimes T_{1}^{R}\right)^{*} \iota_{c l-o p}^{*}\left(z_{1}, \bar{z}_{1}\right)\left(T_{2} v_{1}\right), q_{-\frac{1}{\tau}}^{-\frac{c}{24}}\left(T_{3}^{L} \otimes T_{3}^{R}\right)^{*} \iota_{c l-o p}^{*}\left(z_{2}, \bar{z}_{2}\right)\left(T_{4} v_{2}\right)\right)_{c l} \\
=\sum_{i=1}^{N_{c l}} \operatorname{Tr}_{W_{r_{R}(i)}} e^{-2 \pi i L(0)} \mathcal{Y}_{1}\left(\mathcal{U}\left(q_{-\frac{1}{\tau} s_{1}}\right)\left(-\frac{1}{\tau}\right)^{L(0)} v_{1}, q_{-\frac{1}{\tau} s_{1}}\right) \\
\mathcal{Y}_{2}\left(\mathcal{U}\left(q_{-\frac{1}{\tau} s_{2}}\right)\left(-\frac{1}{\tau}\right)^{L(0)} v_{2}, q_{-\frac{1}{\tau} s_{2}}\right) q_{-\frac{1}{\tau}}^{L(0)-\frac{c}{24}}
\end{array}
$$

where $\mathcal{Y}_{1}$ and $\mathcal{Y}_{2}$ are intertwining operators of types $\left(\begin{array}{c}W_{r_{R}(i)} \\ V_{o p} W_{r_{L}(i)}^{\prime}\end{array}\right)$ and $\left(\begin{array}{c}W_{r_{L}(i)}^{\prime} \\ V_{o p} W_{r_{R}(i)}\end{array}\right)$ respectively and are given by

$$
\begin{aligned}
& \mathcal{Y}_{1}=\sigma_{123}\left(\mathcal{Y}_{l_{c l-o p}^{\prime}}\right) \circ\left(\varphi_{o p} \otimes \operatorname{id}_{W_{r_{L}(i)}^{\prime}}\right), \\
& \mathcal{Y}_{2}=\Omega_{0}\left(\sigma_{132}\left(\mathcal{Y}_{l_{c l-o p}}\right)\right) \circ\left(\varphi_{o p} \otimes \operatorname{id}_{W_{r_{R}(i)}}\right)
\end{aligned}
$$


Proof. Let $z_{3}:=z_{1}-\bar{z}_{1}$ and $z_{4}:=z_{2}-\bar{z}_{2}$. By (2.28) and (3.45), the left hand side of (3.50) equals

$$
\begin{aligned}
& \left(\left(T_{1}^{L} \otimes T_{1}^{R}\right)^{*} \iota_{c l-o p}^{*}\left(z_{1}, \bar{z}_{1}\right)\left(T_{2} v_{1}\right),\left(T_{3}^{L} \otimes T_{3}^{R}\right)^{*} \iota_{c l-o p}^{*}\left(z_{2}, \bar{z}_{2}\right)\left(T_{4} v_{2}\right)\right)_{c l} \\
& =\sum_{i=1}^{N_{c l}} \sum_{\alpha, \beta}\left(\left(T_{3}^{L} \otimes T_{3}^{R}\right)^{*} \iota_{c l-o p}^{*}\left(z_{2}, \bar{z}_{2}\right)\left(T_{4} v_{2}\right), e_{r_{L}(i), \alpha} \otimes e_{r_{R}(i), \beta}\right)_{c l} \\
& \left(\varphi_{c l}^{-1}\left(e_{r_{L}(i), \alpha}^{\prime} \otimes e_{r_{R}(i), \beta}^{\prime}\right),\left(T_{1}^{L} \otimes T_{1}^{R}\right)^{*} \iota_{c l-o p}^{*}\left(z_{1}, \bar{z}_{1}\right)\left(T_{2} v_{1}\right)\right)_{c l} \\
& =\sum_{i=1}^{N_{c l}} \sum_{\alpha, \beta}\left(T_{4} v_{2}, \iota_{c l-o p}\left(z_{2}, \bar{z}_{2}\right)\left(T_{3}^{L} e_{r_{L}(i), \alpha} \otimes T_{3}^{R} e_{r_{R}(i), \beta}\right)\right)_{o p} \\
& \left(\iota_{c l-o p}\left(z_{1}, \bar{z}_{1}\right)\left(\varphi_{c l}^{-1}\left(T_{1}^{L} e_{r_{L}(i), \alpha}^{\prime} \otimes T_{1}^{R} e_{r_{R}(i), \beta}^{\prime}\right)\right), T_{2} v_{1}\right)_{o p} \\
& =\sum_{i=1}^{N_{c l}} \sum_{\alpha, \beta}\left(T_{4} v_{2}, e^{\bar{z}_{2} L(-1)} \mathcal{Y}_{l_{c l-o p}}\left(T_{3}^{L} e_{r_{L}(i), \alpha}, z_{2}-\bar{z}_{2}\right) T_{3}^{R} e_{r_{R}(i), \beta}\right)_{o p} \\
& \left(e^{\bar{z}_{1} L(-1)} \mathcal{Y}_{l_{c l-o p}^{\prime}}\left(T_{1}^{L} e_{r_{L}(i), \alpha}^{\prime}, z_{1}-\bar{z}_{1}\right) T_{1}^{R} e_{r_{R}(i), \beta}^{\prime}, T_{2} v_{1}\right)_{o p} \\
& =\sum_{i=1}^{N_{c l}} \sum_{\alpha, \beta}\left\langle\varphi_{o p}\left(T_{4} v_{2}\right), e^{\bar{z}_{2} L(-1)} \mathcal{Y}_{l_{c l-o p}}\left(T_{3}^{L} e_{r_{L}(i), \alpha}, z_{2}-\bar{z}_{2}\right) T_{3}^{R} e_{r_{R}(i), \beta}\right\rangle \\
& \left\langle e^{\bar{z}_{1} L(-1)} \mathcal{Y}_{l_{c l-o p}^{\prime}}\left(T_{1}^{L} e_{r_{L}(i), \alpha}^{\prime}, z_{1}-\bar{z}_{1}\right) T_{1}^{R} e_{r_{R}(i), \beta}^{\prime}, \varphi_{o p}\left(T_{2} v_{1}\right)\right\rangle \\
& =\sum_{i=1}^{N_{c l}} \sum_{\alpha, \beta}\left\langle e^{-z_{4}^{-1} L(-1)} \sigma_{123}\left(\mathcal{Y}_{l_{c l-o p}}\right)\left(\varphi_{o p}\left(e^{-\bar{z}_{2} L(1)} T_{4} v_{2}\right), z_{4}^{-1}\right)\right. \\
& \left.z_{4}^{-2 L(0)} e^{-z_{4}^{-1} L(1)} T_{3}^{L} e_{r_{L}(i), \alpha}, T_{3}^{R} e_{r_{R}(i), \beta}\right\rangle \\
& \left\langle T_{1}^{R} e_{r_{R}(i), \beta}^{\prime}, e^{-z_{3}^{-1} L(-1)} \sigma_{123}\left(\mathcal{Y}_{l_{c l-o p}^{\prime}}\right)\left(\varphi_{o p}\left(e^{-\bar{z}_{1} L(1)} T_{2} v_{1}\right), z_{3}^{-1}\right)\right. \\
& \left.z_{3}^{-2 L(0)} e^{-z_{3}^{-1} L(1)} T_{1}^{L} e_{r_{L}(i), \alpha}^{\prime}\right\rangle \\
& =\sum_{i=1}^{N_{c l}} \sum_{\alpha, \beta}\left\langle\sigma_{123}\left(\mathcal{Y}_{l_{c l-o p}}\right)\left(\varphi_{o p}\left(e^{-\bar{z}_{2} L(1)} T_{4} v_{2}\right), z_{4}^{-1}\right) z_{4}^{-2 L(0)} e^{-z_{4}^{-1} L(1)} T_{3}^{L} e_{r_{L}(i), \alpha},\right. \\
& \left.e^{z_{4}^{-1} L(1)} T_{3}^{R} e_{r_{R}(i), \beta}\right\rangle\left\langle e_{r_{R}(i), \beta}^{\prime},\left(T_{1}^{R}\right)^{*} e^{-z_{3}^{-1} L(-1)} .\right. \\
& \left.\sigma_{123}\left(\mathcal{Y}_{i_{c l-o p}^{\prime}}\right)\left(\varphi_{o p}\left(e^{-\bar{z}_{1} L(1)} T_{2} v_{1}\right), z_{3}^{-1}\right) z_{3}^{-2 L(0)} e^{-z_{3}^{-1} L(1)} T_{1}^{L} e_{r_{L}(i), \alpha}^{\prime}\right\rangle \\
& =\sum_{i=1}^{N_{c l}} \sum_{\alpha, \beta}\left\langle e_{r_{L}(i), \alpha},\left(T_{3}^{L}\right)^{*} e^{z_{4}^{-1} L(-1)} z_{4}^{-2 L(0)} \tilde{A}_{0} \circ \sigma_{123}\left(\mathcal{Y}_{l_{c l-o p}}\right)\left(e^{-z_{4}^{-1} L(1)} z_{4}^{2 L(0)}\right. \text {. }\right. \\
& \left.\varphi_{o p}\left(e^{-\bar{z}_{2} L(1)} T_{4} v_{2}\right), e^{\pi i} z_{4}\right) e^{z_{4}^{-1} L(1)} T_{3}^{R} e_{r_{R}(i), \beta}\left\langle e_{r_{R}(i), \beta}^{\prime}, \quad\left(T_{1}^{R}\right)^{*} e^{-z_{3}^{-1} L(-1)} .\right. \\
& \left.\sigma_{123}\left(\mathcal{Y}_{i_{c l-o p}^{\prime}}\right)\left(\varphi_{o p}\left(e^{-\bar{z}_{1} L(1)} T_{2} v_{1}\right), z_{3}^{-1}\right) z_{3}^{-2 L(0)} e^{-z_{3}^{-1} L(1)} T_{1}^{L} e_{r_{L}(i), \alpha}^{\prime}\right\rangle
\end{aligned}
$$


We define two intertwining operators as follows:

$$
\begin{aligned}
& \mathcal{Y}_{1}^{(0)}=\sigma_{123}\left(\mathcal{Y}_{i_{c l-o p}^{\prime}}\right) \circ\left(\varphi_{o p} \otimes \operatorname{id}_{W_{r_{L}(i)}^{\prime}}\right), \\
& \mathcal{Y}_{2}^{(0)}=\tilde{A}_{0}\left(\sigma_{123}\left(\mathcal{Y}_{l_{c l-o p}}\right)\right) \circ\left(\varphi_{o p} \otimes \operatorname{id}_{W_{r_{R}(i)}^{\prime}}\right) .
\end{aligned}
$$

Using (3.21),(3.22),(3.23) and (3.24), we further obtain that the left hand side of (3.53) equals

$$
\begin{aligned}
\sum_{i=1}^{N_{c l}} \sum_{\alpha, \beta}\left\langle e_{r_{L}(i), \alpha},\left(\frac{p_{s_{2}}}{r}\right)^{L(0)}\left(\bar{z}_{2}-z_{2}\right)^{L(0)} z_{4}^{-2 L(0)}\right. \\
\mathcal{Y}_{2}^{(0)}\left(z_{4}^{2 L(0)}\left(\bar{z}_{2}-z_{2}\right)^{-L(0)} \mathcal{U}(1)\left(-\frac{1}{\tau}\right)^{L(0)} v_{2}, e^{\pi i} z_{4}\right) \\
\left.\overline{\bar{z}}_{2}-z_{2} L(0) \overline{\left(\frac{p_{s_{2}}}{r}\right)}{ }^{L(0)} e_{r_{R}(i), \beta}\right\rangle\left\langle e_{r_{R}(i), \beta}^{\prime}, \overline{\left(\frac{p_{s_{1}}}{r}\right)}-L(0) \overline{\left(z_{1}-\bar{z}_{1}\right)} L(0)\right. \\
\left.\mathcal{Y}_{2}^{(0)}\left(z_{3}^{-L(0)} \mathcal{U}(1)\left(-\frac{1}{\tau}\right)^{L(0)} v_{1}, z_{3}^{-1}\right) z_{3}^{-L(0)}\left(\frac{p_{s_{1}}}{r}\right)^{-L(0)} e_{r_{L}(i), \alpha}^{\prime}\right\rangle \\
=\sum_{i=1}^{N_{c l}} \operatorname{Tr}_{W_{r_{L}(i)}^{\prime}} \mathcal{Y}_{2}^{(0)}\left(\mathcal{U}\left(p_{s_{2}}\right)(-1 / \tau)^{L(0)} v_{2}, E_{1}\right) E_{2} \mathcal{Y}_{1}^{(0)}\left(\mathcal{U}\left(p_{s_{1}}\right)(-1 / \tau)^{L(0)} v_{1}, p_{s_{1}}\right),
\end{aligned}
$$

where $E_{1}=p_{s_{2}}\left(\bar{z}_{2}-z_{2}\right) z_{4}^{-2} e^{\pi i} z_{4}$ and

$$
E_{2}=p_{s_{2}}^{L(0)}\left(\bar{z}_{2}-z_{2}\right)^{L(0)} z_{4}^{-2 L(0)}{\overline{\left(\bar{z}_{2}-z_{2}\right)}}^{L(0)}{\overline{p_{s_{2}}}}^{L(0)}{\overline{p_{s_{1}}}}^{-L(0)}{\overline{z_{1}-\bar{z}_{1}}}^{L(0)} z_{3}^{-L(0)} p_{s_{1}}^{-L(0)} .
$$

For $E_{1}$, since $z_{4}^{-1}$ is obtained by operations on the intertwining operator where $z_{4}$ is treated formally, $z_{4}^{-1}$ really means $\left|z_{4}\right|^{-1} e^{-i \frac{\pi i}{2}}$. Therefore, we have

$$
E_{1}=p_{s_{2}} e^{\frac{3 \pi i}{2}} e^{-\pi i} e^{\pi i} e^{\frac{\pi i}{2}}=e^{2 \pi i} p_{s_{2}} .
$$

For $E_{2}$, keep in mind (0.1) and (0.2), we have

$$
\begin{aligned}
E_{2} & =\left|\frac{p_{s_{2}}}{p_{s_{1}}}\right|^{2 L(0)}\left(\bar{z}_{2}-z_{2}\right)^{L(0)}\left(z_{2}-\bar{z}_{2}\right)^{-2 L(0)} \frac{\left.\overline{z_{2}}-z_{2}\right)}{(0)} \frac{\overline{\left(z_{1}-\bar{z}_{1}\right)} L(0)}{\left(z_{1}-\bar{z}_{1}\right)^{-L(0)}} \\
& =q_{-\frac{1}{\tau}}^{L(0)} e^{\frac{3 \pi i}{2} L(0)} e^{-2 \frac{\pi i}{2} L(0)} e^{-\frac{3 \pi i}{2} L(0)} e^{-\frac{\pi i}{2} L(0)} e^{-\frac{\pi i}{2} L(0)} \\
& =q_{-\frac{1}{\tau}}^{L(0)} e^{-2 \pi i L(0)}
\end{aligned}
$$

Therefore, we obtain that the left hand side of (3.53) further equals

$$
\begin{gathered}
\sum_{i=1}^{N_{c l}} \operatorname{Tr}_{W_{r_{L}(i)}^{\prime}} \Omega_{0}^{2}\left(\mathcal{Y}_{2}^{(0)}\right)\left(\mathcal{U}\left(p_{s_{2}}\right)\left(-\frac{1}{\tau}\right)^{L(0)} v_{2}, p_{s_{2}}\right) \\
q_{-\frac{1}{\tau}}^{L(0)} e^{-2 \pi i L(0)} \mathcal{Y}_{1}^{(0)}\left(\mathcal{U}\left(p_{s_{1}}\right)\left(-\frac{1}{\tau}\right)^{L(0)} v_{1}, p_{s_{1}}\right),
\end{gathered}
$$


where we have used the fact that $\mathcal{Y}\left(\cdot, e^{2 \pi i} x\right) \cdot=\Omega_{0}^{2}(\mathcal{Y})(\cdot, x) \cdot$ for any intertwining operator $\mathcal{Y}$. By using the property of trace, it is easy to see that (3.58) multiplying $q_{-\frac{1}{\tau}}^{-\frac{c}{24}}$ is nothing but the right hand side of (3.50).

Remark 3.8. It is easy to check that the absolute convergence of the left hand side of (3.53) by our assumption easily implies the absolute convergence of each step in (3.53). Notice that the absolute convergence of the right side of (3.50) is automatic because $V$ is assumed to satisfy the conditions in Theorem 0.1 . Hence, by tracing back the steps in above the proof, we see that the absolute convergence of the left hand side of (3.50) is also automatic.

Now we recall some results in $[\mathrm{H} 9, \mathrm{H} 10]$. We follow the notations in [HKo3]. We denote the unique analytic extension of

$$
\operatorname{Tr}_{W_{a_{1}}} \mathcal{Y}_{a a_{1} ; i}^{a_{1} ;(1)}\left(\mathcal{U}\left(e^{2 \pi i z}\right) w_{a}, e^{2 \pi i z}\right) q_{\tau}^{L(0)-\frac{c}{24}}
$$

in the universal covering space of $1>\left|q_{\tau}\right|>0$ as

$$
E\left(\operatorname{Tr}_{W_{a_{1}}} \mathcal{Y}_{a a_{1} ; i}^{a_{1} ;(1)}\left(\mathcal{U}\left(e^{2 \pi i z}\right) w_{a}, e^{2 \pi i z}\right) q_{\tau}^{L(0)-\frac{c}{24}}\right) .
$$

By [Mi2,H9], above formula is independent of $z$. Consider the map: for $w_{a} \in W_{a}$,

$$
\Psi_{1}\left(\mathcal{Y}_{a a_{1} ; i}^{a_{1} ;(1)}\right): w_{a} \rightarrow E\left(\operatorname{Tr}_{W_{a_{1}}} \mathcal{Y}_{a a_{1} ; i}^{a_{1} ;(1)}\left(\mathcal{U}\left(e^{2 \pi i z}\right) w_{a}, e^{2 \pi i z}\right) q_{\tau}^{L(0)-\frac{c}{24}}\right)
$$

We denote the right hand side of (3.59) as $\Psi_{1}\left(\mathcal{Y}_{a a_{1} ; i}^{a_{1} ;(1)}\right)\left(w_{a} ; z, \tau\right)$. Notice that we choose to add $z$ in the notation even though it is independent of $z$. We define an action of $S L(2, \mathbb{Z})$ on the map (3.59) as follows:

$$
\begin{aligned}
& \left(\left(\begin{array}{ll}
a & b \\
c & d
\end{array}\right)\left(\Psi_{1}\left(\mathcal{Y}_{a a_{1} ; i}^{a_{1} ;(1)}\right)\right)\right)\left(w_{a} ; z, \tau\right) \\
& =E\left(\operatorname{Tr}_{W_{a_{1}}} \mathcal{Y}_{a a_{1} ; i}^{a_{1} ;(1)}\left(\mathcal{U}\left(e^{2 \pi i z^{\prime}}\right)\left(\frac{1}{c \tau+d}\right)^{L(0)} w_{a}, e^{2 \pi i z^{\prime}}\right) q_{\tau^{\prime}}^{L(0)-\frac{c}{24}}\right),
\end{aligned}
$$

where $\tau^{\prime}=\frac{a \tau+b}{c \tau+d}$ and $z^{\prime}=\frac{z}{c \tau+d}$, for $\left(\begin{array}{ll}a & b \\ c & d\end{array}\right) \in S L(2, \mathbb{Z})$ and $w_{a} \in W_{a}$. The following theorem is proved in [Mi2, $\mathrm{H} 7]$.

Theorem 3.9. There exists a unique $A_{a_{2} a_{3}}^{i j} \in \mathbb{C}$ for $a_{2}, a_{3} \in \mathcal{I}$ such that, for $w_{a} \in W_{a}$,

$$
\begin{aligned}
& E\left(\operatorname{Tr}_{W_{a_{1}}} \mathcal{Y}_{a a_{1} ; i}^{a_{1} ;(1)}\left(\mathcal{U}\left(e^{2 \pi i z^{\prime}}\right)\left(\frac{1}{c \tau+d}\right)^{L(0)} w_{a}, e^{2 \pi i z^{\prime}}\right) q_{\tau^{\prime}}^{L(0)-\frac{c}{24}}\right) \\
& =\sum_{a_{3} \in \mathcal{I}} A_{a_{1} a_{2}}^{i j} E\left(\operatorname{Tr}_{W_{a_{1}}} \mathcal{Y}_{a a_{2} ; j}^{a_{2} ;(2)}\left(\mathcal{U}\left(e^{2 \pi i z}\right) w_{a}, e^{2 \pi i z}\right) q_{\tau}^{L(0)-\frac{c}{24}}\right),
\end{aligned}
$$

where $\tau^{\prime}=\frac{a \tau+b}{c \tau+d}$ and $z^{\prime}=\frac{z}{c \tau+d}$. 
In particular, the action of $S=\left(\begin{array}{cc}0 & 1 \\ -1 & 0\end{array}\right)$ on (3.59) induces, for each $a \in \mathcal{I}$, an automorphism on $\oplus_{a_{1} \in \mathcal{I}} \mathcal{V}_{a a_{1}}^{a_{1}}$, denoted as $S(a)$. Namely, we have

$$
S\left(\Psi_{1}\left(\mathcal{Y}_{a a_{1} ; i}^{a_{1} ;(1)}\right)\right)=\Psi_{1}\left(S(a)\left(\mathcal{Y}_{a a_{1} ; i}^{a_{1} ;(1)}\right)\right) .
$$

Combining all such $S(a)$, we obtain an automorphism on $\oplus_{a, a_{1} \in \mathcal{I}} \mathcal{V}_{a a_{1}}^{a_{1}}$. We still denote it as $S$, i.e. $S=\oplus_{a \in \mathcal{I}} S(a)$. Then $S$ can be further extended to a map on $\oplus_{a, a_{3} \in \mathcal{I}} \mathcal{V}_{a a_{3}}^{a_{3}} \otimes \mathcal{V}_{a_{1} a_{2}}^{a}$ given as follows:

$$
S\left(\mathcal{Y}_{a a_{3} ; i}^{a_{3} ;(1)} \otimes \mathcal{Y}_{a_{1} a_{2} ; j}^{a ;(2)}\right):=S\left(\mathcal{Y}_{a a_{3} ; i}^{a_{3} ;(1)}\right) \otimes \mathcal{Y}_{a_{1} a_{2} ; j}^{a ;(2)} .
$$

There is a fusing isomorphism map [H4]:

$$
\mathcal{F}: \oplus_{a \in \mathcal{I}} \mathcal{V}_{a_{1} a}^{a_{4}} \otimes \mathcal{V}_{a_{2} a_{3}}^{a} \stackrel{\cong}{\rightarrow} \oplus_{b \in \mathcal{I}} \mathcal{V}_{b a_{3}}^{a_{4}} \otimes \mathcal{V}_{a_{1} a_{2}}^{b}
$$

for $a_{1}, a_{2}, a_{3}, a_{4} \in \mathcal{I}$. Using the isomorphism $\mathcal{F}$, we obtain a natural action of $S$ on $\oplus_{b, a_{3}} \mathcal{V}_{a_{1} b}^{a_{3}} \otimes \mathcal{V}_{a_{2} a_{3}}^{b}$

It is shown in [H7] that the following 2-points genus-one correlation function, for $a_{1}, a_{2}, a_{3}, a_{4} \in \mathcal{I}, i=1, \ldots, N_{a a_{1}}^{a_{1}}, j=1, \ldots, N_{a_{2} a_{3}}^{a}$ and $w_{a_{k}} \in W_{a_{k}}, k=2,3$,

$$
\operatorname{Tr}_{W_{a_{1}}} \mathcal{Y}_{a a_{1} ; i}^{a_{1} ;(1)}\left(\mathcal{U}\left(q_{z_{2}}\right) \mathcal{Y}_{a_{2} a_{3} ; j}^{a ;(2)}\left(w_{a_{2}}, z_{1}-z_{2}\right) w_{a_{3}}, q_{z_{2}}\right) q_{\tau}^{L(0)-\frac{c}{24}}
$$

is absolutely convergent when $1>\left|e^{2 \pi i z_{2}}\right|>\left|q_{\tau}\right|>0$ and $1>\left|e^{2 \pi i\left(z_{1}-z_{2}\right)}\right|>0$ and single-valued in the chosen branch. It can be extended uniquely to a single-valued analytic function on the universal covering space of

$$
M_{1}^{2}=\left\{\left(z_{1}, z_{2}, \tau\right) \in \mathbb{C}^{3} \mid z_{1} \neq z_{2}+p \tau+q, \forall p, q \in Z, \tau \in \mathbb{H}\right\} .
$$

This universal covering space is denoted by $\tilde{M}_{1}^{2}$. We denote this single-valued analytic function on $\tilde{M}_{1}^{2}$ as

$$
E\left(\operatorname{Tr}_{W_{a_{1}}} \mathcal{Y}_{a a_{1} ; i}^{a_{1} ;(1)}\left(\mathcal{U}\left(q_{z_{2}}\right) \mathcal{Y}_{a_{2} a_{3} ; j}^{a ;(2)}\left(w_{a_{2}}, z_{1}-z_{2}\right) w_{a_{3}}, q_{z_{2}}\right) q_{\tau}^{L(0)-\frac{c}{24}}\right) .
$$

We denote the space spanned by such functions on $\tilde{M}_{1}^{2}$ by $\mathbb{G}_{1 ; 2}$.

For $\mathcal{Y}_{a a_{1} ; i}^{a_{1} ;(1)} \in \mathcal{V}_{a a_{1}}^{a_{1}}$ and $\mathcal{Y}_{a_{2} a_{3} ; j}^{a ;(2)} \in \mathcal{V}_{a_{2} a_{3}}^{a}$, we now define the following linear map:

$$
\Psi_{2}\left(\mathcal{Y}_{a a_{1} ; i}^{a_{1} ;(1)} \otimes \mathcal{Y}_{a_{2} a_{3} ; j}^{a ;(2)}\right): \quad \oplus_{b_{2}, b_{3} \in \mathcal{I}} W_{b_{2}} \otimes W_{b_{3}} \rightarrow \mathbb{G}_{1 ; 2}
$$

as follows: the map restricted on $W_{b_{2}} \otimes W_{b_{3}}$ is defined by 0 for $b_{2} \neq a_{2}$ or $b_{3} \neq a_{3}$, and by

$$
E\left(\operatorname{Tr}_{W_{a_{1}}} \mathcal{Y}_{a a_{1} ; i}^{a_{1} ;(1)}\left(\mathcal{U}\left(q_{z_{2}}\right) \mathcal{Y}_{a_{2} a_{3} ; j}^{a ;(2)}\left(w_{a_{2}}, z_{1}-z_{2}\right) w_{a_{3}}, q_{z_{2}}\right) q_{\tau}^{L(0)-\frac{c}{24}}\right),
$$

for all $w_{a_{k}} \in W_{a_{k}}, k=2,3$. The following identity was proved in [H9]:

$$
\begin{aligned}
& \left(\Psi_{2}\left(\mathcal{Y}_{a a_{1} ; i}^{a_{1} ;(1)} \otimes \mathcal{Y}_{a_{2} a_{3} ; j}^{a ;(2)}\right)\left(\left(-\frac{1}{\tau}\right)^{L(0)} w_{a_{2}} \otimes\left(-\frac{1}{\tau}\right)^{L(0)} w_{a_{3}}\right)\right)\left(-\frac{1}{\tau} z_{1},-\frac{1}{\tau} z_{2} ;-\frac{1}{\tau}\right) \\
& =\left(\Psi_{2}\left(S\left(\mathcal{Y}_{a a_{1} ; i}^{a_{1} ;(1)} \otimes \mathcal{Y}_{a_{2} a_{3} ; j}^{a ;(2)}\right)\right)\left(w_{a_{2}} \otimes w_{a_{3}}\right)\right)\left(z_{1}, z_{2}, \tau\right) .
\end{aligned}
$$


One can also produce 2-point genus-one correlation functions from a product of two intertwining operators. It is proved in [H7] that $\forall w_{a_{k}} \in W_{a_{k}}, k=1,2$,

$$
\operatorname{Tr}_{W_{a_{4}}} \mathcal{Y}_{a_{1} a_{3} ; i}^{a_{4} ;(1)}\left(\mathcal{U}\left(q_{z_{1}}\right) w_{a_{1}}, q_{z_{1}}\right) \mathcal{Y}_{a_{2} a_{4} ; j}^{a_{3} ;(2)}\left(\mathcal{U}\left(q_{z_{2}}\right) w_{a_{2}}, q_{z_{2}}\right) q_{\tau}^{L(0)-\frac{c}{24}},
$$

is absolutely convergent when $1>\left|q_{z_{1}}\right|>\left|q_{z_{2}}\right|>\left|q_{\tau}\right|>0$. Equation (3.68) has a unique extension to the universal covering space $\tilde{M}_{1}^{2}$, denoted as

$$
E\left(\operatorname{Tr}_{W_{a_{4}}} \mathcal{Y}_{a_{1} a_{3} ; i}^{a_{4} ;(1)}\left(\mathcal{U}\left(q_{z_{1}}\right) w_{a_{1}}, q_{z_{1}}\right) \mathcal{Y}_{a_{2} a_{4} ; j}^{a_{3} ;(2)}\left(\mathcal{U}\left(q_{z_{2}}\right) w_{a_{2}}, q_{z_{2}}\right) q_{\tau}^{L(0)-\frac{c}{24}}\right)
$$

Such functions on $\tilde{M}_{1}^{2}$ also span $\mathbb{G}_{1 ; 2}$. We define a map

$$
\Psi_{2}\left(\mathcal{Y}_{a_{1} a_{3} ; i}^{a_{4} ;(1)} \otimes \mathcal{Y}_{a_{2} a_{4} ; j}^{a_{3} ;(2)}\right): \oplus_{b_{1}, b_{2} \in \mathcal{I}} W_{b_{1}} \otimes W_{b_{2}} \rightarrow \mathbb{G}_{1 ; 2}
$$

as follows: the map restricted on $W_{b_{1}} \otimes W_{b_{2}}$ is defined by 0 for $b_{1} \neq a_{1}, b_{2} \neq a_{2}$, and by (3.69) for $w_{a_{1}} \in W_{a_{1}}, w_{a_{2}} \in W_{a_{2}}$.

It was proved by Huang in [H7] that the fusing isomorphism (3.64) gives the following associativity:

$$
\begin{aligned}
E( & \left.\operatorname{Tr}_{W_{a_{4}}} \mathcal{Y}_{a_{1} a_{3} ; i}^{a_{4} ;(1)}\left(\mathcal{U}\left(q_{z_{1}}\right) w_{a_{1}}, q_{z_{1}}\right) \mathcal{Y}_{a_{2} a_{4} ; j}^{a_{3} ;(2)}\left(\mathcal{U}\left(q_{z_{2}}\right) w_{a_{2}}, q_{z_{2}}\right) q_{\tau}^{L(0)-\frac{c}{24}}\right) \\
= & \sum_{a_{5} \in \mathcal{I}} \sum_{k, l} F\left(\mathcal{Y}_{a_{1} a_{3} ; i}^{a_{4} ;(1)} \otimes \mathcal{Y}_{a_{2} a_{4} ; j}^{a_{3} ;(2)}, \mathcal{Y}_{a_{5} a_{4} ; k}^{a_{4} ;(3)} \otimes \mathcal{Y}_{a_{1} a_{2} ; l}^{a_{5} ;(4)}\right) \\
& E\left(\operatorname{Tr}_{W_{a_{4}}} \mathcal{Y}_{a_{5} a_{4} ; k}^{a_{4} ;(3)}\left(\mathcal{U}\left(q_{z_{2}}\right) \mathcal{Y}_{a_{1} a_{2} ; l}^{a_{5} ;(4)}\left(w_{a_{1}}, z_{1}-z_{2}\right) w_{a_{2}}, q_{z_{2}}\right) q_{\tau}^{L(0)-\frac{c}{24}}\right),
\end{aligned}
$$

where $F\left(\mathcal{Y}_{a_{1} a_{3} ; i}^{a_{4} ;(1)} \otimes \mathcal{Y}_{a_{2} a_{4} ; j}^{a_{3} ;(2)}, \mathcal{Y}_{a_{5} a_{4} ; k}^{a_{4} ;(3)} \otimes \mathcal{Y}_{a_{1} a_{2} ; l}^{a_{5} ;(4)}\right)$ is the matrix representation of $\mathcal{F}$ in the basis $\left\{\mathcal{Y}_{a_{1} a_{3} ; i}^{a_{4} ;(1)} \otimes \mathcal{Y}_{a_{2} a_{4} ; j}^{a_{3} ;(2)}\right\}_{i, j},\left\{\mathcal{Y}_{a_{5} a_{4} ; k}^{a_{4} ;(3)} \otimes \mathcal{Y}_{a_{1} a_{2} ; l}^{a_{5} ;(4)}\right\}_{k, l}$. Therefore, $\forall w_{a_{k}} \in W_{a_{k}}, k=1,2$, we obtain

$$
\begin{gathered}
\left(\Psi_{2}\left(\mathcal{Y}_{a_{1} a ; i}^{a_{3} ;(1)} \otimes \mathcal{Y}_{a_{2} a_{3} ; j}^{a ;(2)}\right)\left(\left(-\frac{1}{\tau}\right)^{L(0)} w_{a_{1}} \otimes\left(-\frac{1}{\tau}\right)^{L(0)} w_{a_{2}}\right)\right)\left(-\frac{1}{\tau} z_{1},-\frac{1}{\tau} z_{2} ;-\frac{1}{\tau}\right) \\
=\left(\Psi_{2}\left(S\left(\mathcal{Y}_{a_{1} a ; i}^{a_{3} ;(1)} \otimes \mathcal{Y}_{a_{2} a_{3} ; j}^{a ;(2)}\right)\right)\left(w_{a_{1}} \otimes w_{a_{2}}\right)\right)\left(z_{1}, z_{2}, \tau\right) .
\end{gathered}
$$

Combining (3.49), (3.50), (3.51), (3.52) and (3.72) and Remark 3.8, we obtain a simpler version of the Cardy condition.

Theorem 3.10. The Cardy condition can be rewritten as follows:

$$
\begin{gathered}
\left(\theta_{W_{r_{R}(i)}} \circ \sigma_{123}\left(\mathcal{Y}_{i_{c l-o p}^{\prime}}\right) \circ\left(\varphi_{o p} \otimes \operatorname{id}_{W_{r_{L}(i)}^{\prime}}\right)\right) \otimes\left(\Omega_{0}\left(\sigma_{132}\left(\mathcal{Y}_{l_{c l-o p}}\right)\right) \circ\left(\varphi_{o p} \otimes \operatorname{id}_{W_{r_{R}(i)}}\right)\right) \\
=S^{-1}\left(Y_{o p}^{f} \otimes\left(\Omega_{-1}\left(Y_{o p}^{f}\right) \circ\left(\theta_{V_{o p}} \otimes \operatorname{id}_{V_{o p}}\right)\right)\right) .
\end{gathered}
$$

\section{Modular Tensor Categories}

This section is independent of the rest of this work. The tensor product theory of modules over a vertex operator algebra has been developed by Huang and Lepowsky [HL1][HL4,H2]. In particular, the notion of vertex tensor category is introduced in [HL1]. Huang later proved in [H11] that $\mathcal{C}_{V}$ is a modular tensor category for $V$ satisfying conditions in Theorem 0.1. In Sect. 4.1, we review some basic ingredients of the modular tensor category $\mathcal{C}_{V}$. In Sect. 4.2, we show how to find in $\mathcal{C}_{V}$ a graphical representation of the modular transformation $S: \tau \mapsto-\frac{1}{\tau}$ discussed in Sect. 3.2. 
4.1. Preliminaries. We recall some ingredients of vertex tensor category $\mathcal{C}_{V}$ and those of modular tensor category structure on $\mathcal{C}_{V}$ constructed in [H11].

There is an associativity isomorphism $\mathcal{A}$,

$$
\mathcal{A}: W_{1} \otimes\left(W_{2} \otimes W_{3}\right) \rightarrow\left(W_{1} \otimes W_{2}\right) \otimes W_{3},
$$

for each triple of $V$-modules $W_{1}, W_{2}, W_{3}$. The relation between the fusing isomorphism $\mathcal{F}$ (recall (3.64)) and the associativity isomorphism $\mathcal{A}$ in $\mathcal{C}_{V}$ is described by the following commutative diagram:

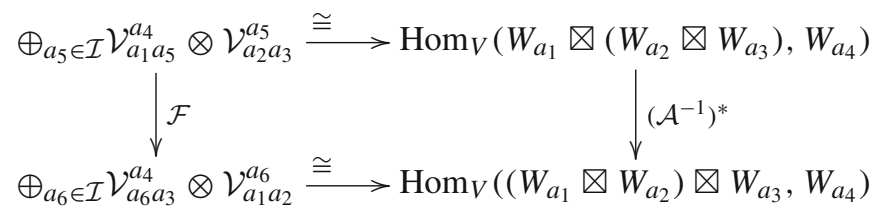

where the two horizontal maps are canonical isomorphisms induced from the universal property of $\otimes$.

We recall the braiding structure on $\mathcal{C}_{V}$. For each pair of $V$-modules $W_{1}, W_{2}$, there is also a natural isomorphism, for $z>0, \mathcal{R}_{+}^{P(z)}: W_{1} \otimes_{P(z)} W_{2} \rightarrow W_{2} \bigotimes_{P(z)} W_{1}$, defined by

$$
\overline{\mathcal{R}_{+}^{P(z)}}\left(w_{1} \otimes_{P(z)} w_{2}\right)=e^{L(-1)} \overline{\mathcal{T}}_{\gamma_{+}}\left(w_{2} \otimes_{P(-z)} w_{1}\right),
$$

where $\gamma_{+}$is a path from $-z$ to $z$ inside the lower half plane as shown in the following graph

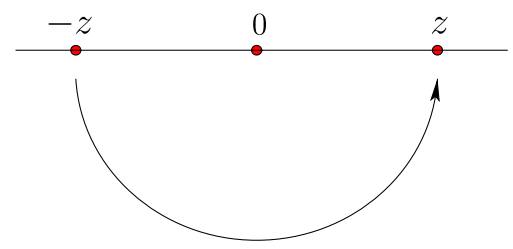

The inverse of $\mathcal{R}_{+}^{P(z)}$ is denoted by $\mathcal{R}_{-}^{P(z)}$, which is characterized by

$$
\overline{\mathcal{R}_{-}^{P(z)}}\left(w_{2} \otimes_{P(z)} w_{1}\right)=e^{L(-1)} \overline{\mathcal{T}}_{\gamma_{-}}\left(w_{1} \otimes_{P(-z)} w_{2}\right),
$$

where $\gamma_{-}$is a path in the upper half plane as shown in the following graph

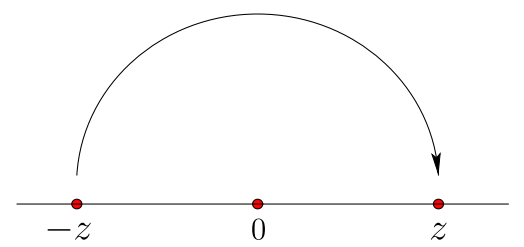

We denote $\mathcal{R}_{ \pm}^{P(1)}$ simply as $\mathcal{R}_{ \pm}$. The natural isomorphisms $\mathcal{R}_{ \pm}$give $\mathcal{C}_{V}$ two different braiding structures. We choose $\mathcal{R}_{+}$as the default braiding structure on $\mathcal{C}_{V}$. Sometimes, we will denote it by $\left(\mathcal{C}_{V}, \mathcal{R}_{+}\right)$to emphasize our choice of braiding isomorphisms.

Notice that our choice of $\mathcal{R}_{ \pm}$follows that in [Ko2], which is different from that in $[\mathrm{H} 8, \mathrm{H} 11, \mathrm{Ko} 1]$. For each $V$-module $W,(3.39)$ defines an automorphism $\theta_{W}: W \rightarrow W$ 
called a twist. A $V$-module $W$ is said to have a trivial twist if $\theta_{W}=\operatorname{id}_{W}$. The twist $\theta$ and braiding $\mathcal{R}_{+}$satisfy the following three balancing axioms

$$
\begin{aligned}
\theta_{W_{1}} \nabla_{W_{2}} & =\mathcal{R}_{+} \circ \mathcal{R}_{+} \circ\left(\theta_{W_{1}} \otimes \theta_{W_{2}}\right), \\
\theta_{V} & =\operatorname{id}_{V}, \\
\theta_{W^{\prime}} & =\left(\theta_{W}\right)^{*},
\end{aligned}
$$

for any pair of $V$-modules $W_{1}, W_{2}$.

Let $\left\{\mathcal{Y}_{e a}^{a}\right\}$ be a basis of $\mathcal{V}_{e a}^{a}$ for all $a \in \mathcal{I}$ such that it coincides with the vertex operator $Y_{W_{a}}$, which defines the $V$-module structure on $W_{a}$, i.e. $\mathcal{Y}_{e a}^{a}=Y_{W_{a}}$. We choose a basis $\left\{\mathcal{Y}_{a e}^{a}\right\}$ of $\mathcal{V}_{a e}^{a}$ as follows:

$$
\mathcal{Y}_{a e}^{a}=\Omega_{-1}\left(\mathcal{Y}_{e a}^{a}\right)
$$

We also choose a basis $\left\{\mathcal{Y}_{a a^{\prime}}^{e}\right\}$ of $\mathcal{V}_{a a^{\prime}}^{e}$ as

$$
\mathcal{Y}_{a a^{\prime}}^{e}=\mathcal{Y}_{a a^{\prime}}^{e^{\prime}}=\hat{A}_{0}\left(\mathcal{Y}_{a e}^{a}\right)=\sigma_{132}\left(\mathcal{Y}_{e a}^{a}\right) \text {. }
$$

Notice that these choices are made for all $a \in \mathcal{I}$. In particular, we have

$$
\mathcal{Y}_{a^{\prime} e}^{a^{\prime}}=\Omega_{-1}\left(\mathcal{Y}_{e a^{\prime}}^{a^{\prime}}\right), \quad \mathcal{Y}_{a^{\prime} a}^{e}=\mathcal{Y}_{a^{\prime} a}^{e^{\prime}}=\hat{A}_{0}\left(\mathcal{Y}_{a^{\prime} e}^{a^{\prime}}\right)
$$

The following relation was proved in [Ko1].

$$
\mathcal{Y}_{a^{\prime} a}^{e}=e^{2 \pi i h_{a}} \Omega_{0}\left(\mathcal{Y}_{a a^{\prime}}^{e}\right)=e^{-2 \pi i h_{a}} \Omega_{-1}\left(\mathcal{Y}_{a a^{\prime}}^{e}\right) \text {. }
$$

For any $V$-modules $W_{1}, W_{2}, W_{3}$ and $\mathcal{Y} \in \mathcal{V}_{W_{1} W_{2}}^{W_{3}}$, we denote by $m_{\mathcal{Y}}$ the morphism in $\operatorname{Hom}_{V}\left(W_{1} \otimes W_{2}, W_{3}\right)$ associated to $\mathcal{Y}$ under the identification of two spaces induced by the universal property of $\otimes$.

Now we recall the construction of duality maps [H11]. We will follow the convention in [Ko1]. Since $\mathcal{C}_{V}$ is semisimple, we only need to discuss irreducible modules. For $a \in \mathcal{I}$, the right duality maps $e_{a}: W_{a}^{\prime} \otimes W_{a} \rightarrow V$ and $i_{a}: V \rightarrow W_{a} \otimes W_{a}^{\prime}$ for $a \in \mathcal{I}$ are given by

$$
e_{a}=m_{\mathcal{Y}_{a^{\prime} a}^{e}}^{e}, \quad m_{\mathcal{Y}_{a a^{\prime}}^{e}}^{e} \circ i_{a}=\operatorname{dim} a \operatorname{id}_{V}
$$

where $\operatorname{dim} a \neq 0$ for $a \in \mathcal{I}$ (proved by Huang in [H10]). The left duality maps $e_{a}^{\prime}$ : $W^{a} \otimes W_{a}^{\prime} \rightarrow V$ and $i_{a}^{\prime}: V \rightarrow W_{a}^{\prime} \otimes W^{a}$ are given by

$$
e_{a}^{\prime}=m_{\mathcal{Y}_{a a^{\prime}}^{e}}, \quad m_{\mathcal{Y}_{a^{\prime} a}^{e}} \circ i_{a}^{\prime}=\operatorname{dim} a \operatorname{id}_{V} .
$$

In a ribbon category, there is a powerful tool called graphic calculus. One can express various morphisms in terms of graphs. In particular, the right duality maps $i_{a}$ and $e_{a}$ are denoted by the following graphs:

$$
i_{a}=\int^{a} \int_{a}^{a^{\prime}} \quad e_{a}=\overbrace{a^{\prime},}
$$


the left duality maps are denoted by

$$
i_{a}^{\prime}=\operatorname{a}^{\prime}\left(\left.e_{a}^{a} \quad e_{a}^{\prime}\right|_{a}\right.
$$

and the twist and its inverse, for any object $W$, are denoted by

$$
\theta_{W}=\left.\right|_{W} ^{W} \quad \theta_{W}^{-1}=b_{W}^{W}
$$

The identity (4.11) proved in [Ko2] is nothing but the following identity:

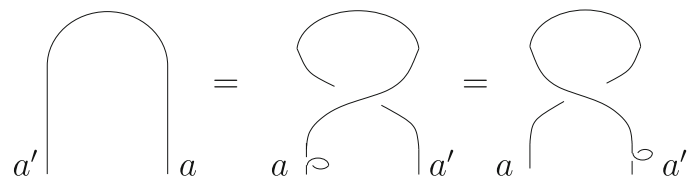

This formula (4.12) is implicitly used in many graphic calculations in this work.

A basis $\left\{\mathcal{Y}_{a_{1} a_{2} ; i}^{a_{3} ;(1)}\right\}_{i=1}^{N_{a_{1} a_{2}}^{a_{3}}}$ of $\mathcal{V}_{a_{1} a_{2}}^{a_{3}}$ for $a_{1}, a_{2}, a_{3} \in \mathcal{I}$ induces a basis $\left\{e_{a_{1} a_{2} ; i}^{a_{3}}\right\}$ of Hom $\left(W_{a_{1}} \otimes W_{a_{2}}, W_{a_{3}}\right)$. One can also denote $e_{a_{1} a_{2} ; i}^{a_{3}}$ as the following graph:

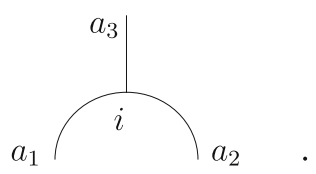

Note that we will always use $a$ to represent $W_{a}$ and $a^{\prime}$ to represent $W_{a}^{\prime}$ in graphs for simplicity. By the universal property of $\otimes_{P(z)}$, the map $\Omega_{0}: \mathcal{V}_{a_{1} a_{2}}^{a_{3}} \rightarrow \mathcal{V}_{a_{2} a_{1}}^{a_{3}}$ induces a linear map $\Omega_{0}: \operatorname{Hom}_{V}\left(W_{a_{1}} \otimes W_{a_{2}}, W_{a_{3}}\right) \rightarrow \operatorname{Hom}_{V}\left(W_{a_{2}} \otimes W_{a_{1}}, W_{a_{3}}\right)$ given as follows:



Let us choose a basis $\left\{f_{a_{3} ; j}^{a_{1} a_{2}}\right\}_{j=1}^{N_{a_{1} a_{2}}^{a_{3}}}$ of $\operatorname{Hom}_{V}\left(W_{a_{3}}, W_{a_{1}} \otimes W_{a_{2}}\right)$, denoted by

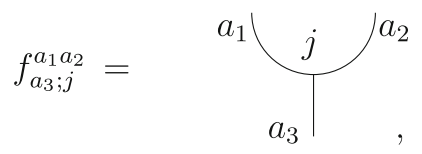

such that

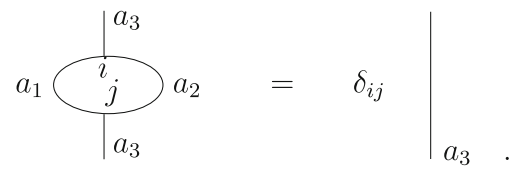


The following identity is proved in [Ko1]

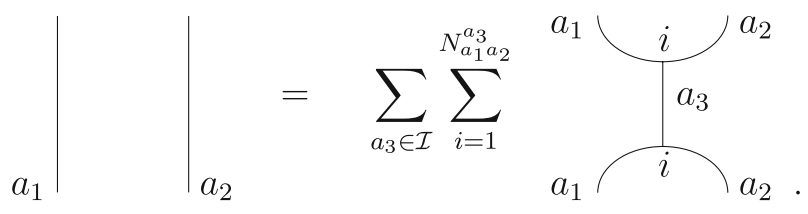

We prove a similar identity below.

\section{Lemma 4.1.}

$$
\sum_{a_{4} \in \mathcal{I}} \sum_{l} \frac{\operatorname{dim} a_{4}}{\operatorname{dim} b} \overbrace{a_{3}^{\prime}}^{a_{3}^{\prime}} \overbrace{\frac{k}{k}}^{\frac{b}{k}} a_{4}=\left.\left.\right|_{a_{3}^{\prime}}\right|_{b}
$$

Proof. Using the first balancing axiom (4.6), we have



Then the lemma follows from the following relations:

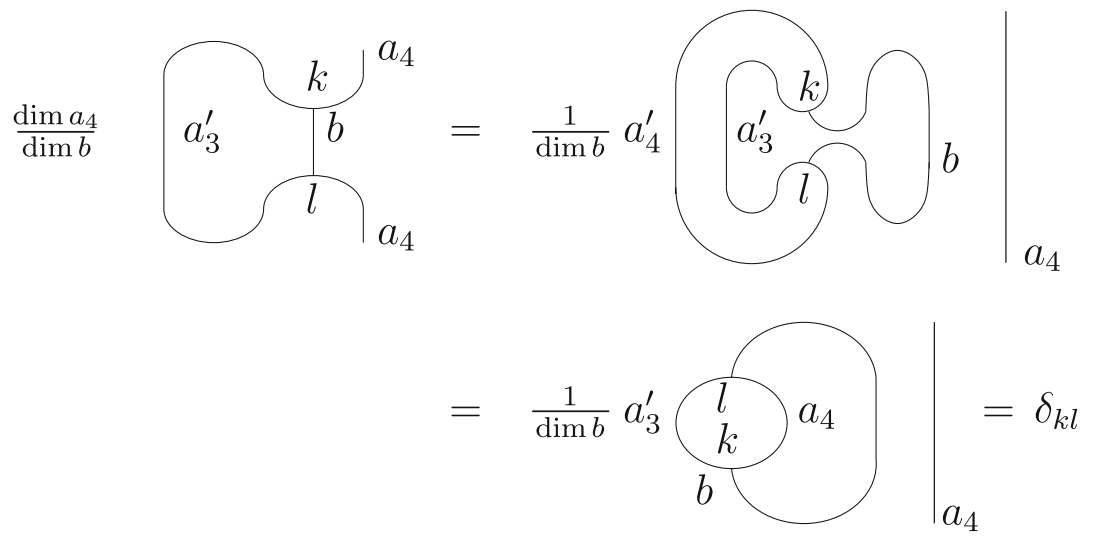

Similar to $\Omega_{0}, \tilde{A}_{0}, \sigma_{123}$ and $\sigma_{132}$ can also be described graphically as proved in [Ko1]. We recall these results below. 


\section{Proposition 4.2.}

$\tilde{A}_{0}:$<smiles>CCC(C)CC</smiles>
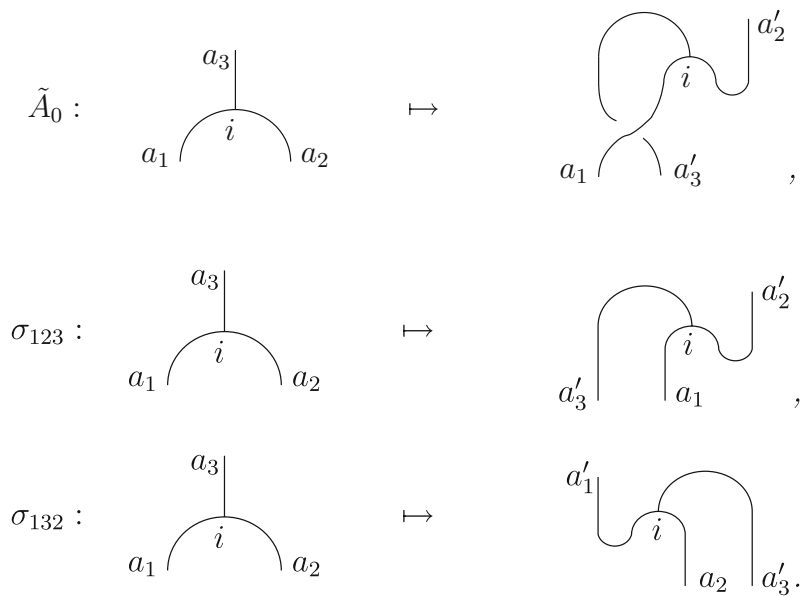

4.2. Graphical representation of $S: \tau \mapsto-\frac{1}{\tau}$. In [HKo3], we defined an action of $\alpha, \beta$ on $\Psi_{2}\left(\mathcal{Y}_{a a_{1} ; p}^{a_{1} ;(1)} \otimes \mathcal{Y}_{a_{2} a_{3} ; q}^{a ;(2)}\right)$. More precisely,

$$
\begin{aligned}
& \alpha\left(\Psi_{2}\left(\mathcal{Y}_{a a_{1} ; p}^{a_{1} ;(1)} \otimes \mathcal{Y}_{a_{2} a_{3} ; q}^{a ;(2)}\right)\right): \oplus_{a_{2}, a_{3} \in \mathcal{I}} W_{a_{2}} \otimes W_{a_{3}} \rightarrow \mathbb{G}_{1 ; 2}, \\
& \beta\left(\Psi_{2}\left(\mathcal{Y}_{a a_{1} ; p}^{a_{1} ;(1)} \otimes \mathcal{Y}_{a_{2} a_{3} ; q}^{a ;(2)}\right)\right): \bigoplus_{a_{2}, a_{3} \in \mathcal{I}} W_{a_{2}} \otimes W_{a_{3}} \rightarrow \mathbb{G}_{1 ; 2}
\end{aligned}
$$

are defined by

$$
\begin{aligned}
(\alpha & \left(\Psi_{2}\left(\mathcal{Y}_{a a_{1} ; p}^{a_{1} ;(1)} \otimes \mathcal{Y}_{a_{2} a_{3} ; q}^{a ;(2)}\right)\right)\left(w_{2} \otimes w_{3}\right) \\
= & \left(\Psi_{2}\left(\mathcal{Y}_{a a_{1} ; p}^{a_{1} ;(1)} \otimes \mathcal{Y}_{a_{2} a_{2}^{\prime} ; q}^{a ;(2)}\right)\right)\left(w_{2} \otimes w_{3} ; z_{1}, z_{2}-1 ; \tau\right), \\
& \left(\beta\left(\Psi\left(\mathcal{Y}_{a a_{1} ; p}^{a_{1} ;(1)} \otimes \mathcal{Y}_{a_{2} ;(2)}^{a ;(2)}\right)\right)\right)\left(w_{2} \otimes w_{3}\right) \\
= & \left(\Psi_{2}\left(\mathcal{Y}_{a a_{1} ; p}^{a_{1} ;(1)} \otimes \mathcal{Y}_{a_{2} a_{3} ; q}^{a ;(2)}\right)\right)\left(w_{2} \otimes w_{3} ; z_{1}, z_{2}+\tau ; \tau\right)
\end{aligned}
$$

if $w_{2} \otimes w_{3} \in W_{a_{2}} \otimes W_{a_{3}}$, and by 0 if otherwise.

We also showed in [HKo3] that $\alpha$ induces an automorphism on $\oplus_{a \in \mathcal{I}} \mathcal{V}_{a a_{1}}^{a_{1}} \otimes \mathcal{V}_{a_{2} a_{3}}^{a}$ given as follows:

$$
\begin{aligned}
\mathcal{Y}_{a a_{1} ; i}^{a_{1} ;(1)} \otimes \mathcal{Y}_{a_{2} a_{3} ; j}^{a ;(2)} \mapsto & \sum_{b, c \in \mathcal{A}} \sum_{k, l, p, q} e^{-2 \pi i h_{a_{3}} F^{-1}}\left(\mathcal{Y}_{a a_{1} ; i}^{a_{1} ;(1)} \otimes \mathcal{Y}_{a_{2} a_{3} ; j}^{a ;(2)} ; \mathcal{Y}_{a_{2} b ; k}^{a_{1} ;(3)} \otimes \mathcal{Y}_{a_{3} a_{1} ; l}^{b ;(4)}\right) \\
& F\left(\mathcal{Y}_{a_{2} b ; k}^{a_{1} ;(3)} \otimes \Omega_{-1}^{2}\left(\mathcal{Y}_{a_{3} a_{1} ; l}^{b ;(4)}\right) ; \mathcal{Y}_{c a_{1} ; p}^{a_{1} ;(5)} \otimes \mathcal{Y}_{a_{2} a_{3} ; q}^{c ;(6)}\right) \\
& \mathcal{Y}_{c a_{1} ; p}^{a_{1} ;(5)} \otimes \mathcal{Y}_{a_{2} a_{3} ; q}^{c ;(6)}
\end{aligned}
$$

We still denote this automorphism and its natural extension on $\oplus_{a, a_{1} \in \mathcal{I}} \mathcal{V}_{a a_{1}}^{a_{1}} \otimes \mathcal{V}_{a_{2} a_{3}}^{a}$ by $\alpha$. The following lemma follows immediately from (4.28). 
Lemma 4.3. $\alpha$ can also be expressed graphically as follows:
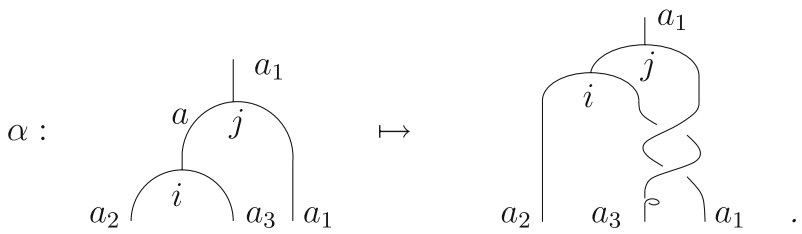

For $\beta$, we prefer to use maps $\tilde{A}_{0}$ and $\hat{A}_{0}$ defined in (3.35) instead of the map $A_{0}$ used in [HKo3]. We obtain the following lemma, which is proved in the Appendix.

Lemma 4.4. $\beta$ also induces an automorphism on $\oplus_{a, a_{1} \in \mathcal{I}} \mathcal{V}_{a a_{1}}^{a_{1}} \otimes \mathcal{V}_{a_{2} a_{3}}^{a}$ given by

$$
\begin{aligned}
\mathcal{Y}_{a a_{1} ; i}^{a_{1} ;(1)} \otimes \mathcal{Y}_{a_{2} a_{3} ; j}^{a ;(2)} \mapsto & \sum_{b \in \mathcal{I}} \sum_{k, l} \sum_{c \in \mathcal{I}} \sum_{p, q} F^{-1}\left(\mathcal{Y}_{a a_{1} ; i}^{a_{1} ;(1)} \otimes \mathcal{Y}_{a_{2} a_{3} ; j}^{a ;(2)} ; \mathcal{Y}_{a_{2} b ; k}^{a_{1} ;(3)} \otimes \mathcal{Y}_{a_{3} a_{1} ; l}^{b ;(4)}\right) \\
& F\left(\tilde{A}_{0}\left(\mathcal{Y}_{a_{2} b ; k}^{a_{1} ;(3)}\right) \otimes \tilde{A}_{0}\left(\mathcal{Y}_{a_{3} a_{1} ; l}^{b ;(4)}\right), \mathcal{Y}_{c b^{\prime} ; p}^{b^{\prime} ;(5)} \otimes \mathcal{Y}_{a_{2} a_{3} ; q}^{c ;(6)}\right) \\
& \hat{A}_{0}\left(\mathcal{Y}_{c b^{\prime} ; p}^{b^{\prime} ;(5)}\right) \otimes \Omega_{0}^{2}\left(\mathcal{Y}_{a_{2} a_{3} ; q}^{c ;(6)}\right)
\end{aligned}
$$

We still denote this automorphism by $\beta$.

Lemma 4.5. $\beta$ can be expressed graphically as follows:
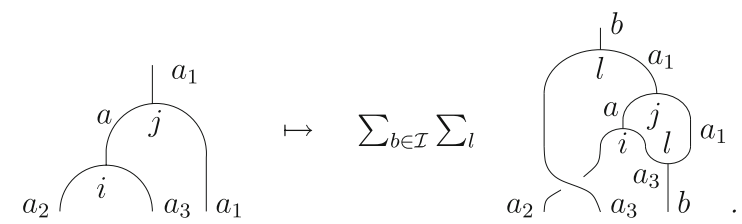

Proof. Using (4.30), we can see that $\beta$ is the composition of following maps

$$
\begin{aligned}
\oplus_{a, a_{1} \in \mathcal{I}} \mathcal{V}_{a a_{1}}^{a_{1}} \otimes \mathcal{V}_{a_{2} a_{3}}^{a} \stackrel{\mathcal{F}^{-1}}{\longrightarrow} \oplus_{b, a_{1} \in \mathcal{I}} \mathcal{V}_{a_{2} b}^{a_{1}} \otimes \mathcal{V}_{a_{3} a_{1}}^{b} \stackrel{\tilde{A}_{0} \otimes \tilde{A}_{0}}{\longrightarrow} \oplus_{b, a_{1} \in \mathcal{I}} \mathcal{V}_{a_{2} a_{1}^{\prime}}^{b^{\prime}} \otimes \mathcal{V}_{a_{3} b^{\prime}}^{a_{1}^{\prime}} \\
\bigoplus_{b, c \in \mathcal{I}} \mathcal{V}_{c b}^{b} \otimes \mathcal{V}_{a_{2} a_{3}}^{c} \stackrel{\mathcal{F}}{\stackrel{\hat{A}_{0} \otimes \Omega_{0}^{2}}{r}} \oplus_{b, c \in \mathcal{I}} \mathcal{V}_{c b^{\prime}}^{b^{\prime}} \otimes \mathcal{V}_{a_{2} a_{3}}^{c}
\end{aligned}
$$


By the commutative diagram (4.1), (4.32) can be rewritten graphically as follows:

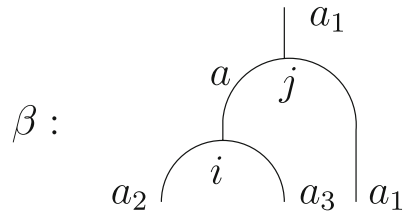

$$
=\sum_{b \in T} \sum_{k}
$$

$\mapsto \sum_{b \in T} \sum_{k}$

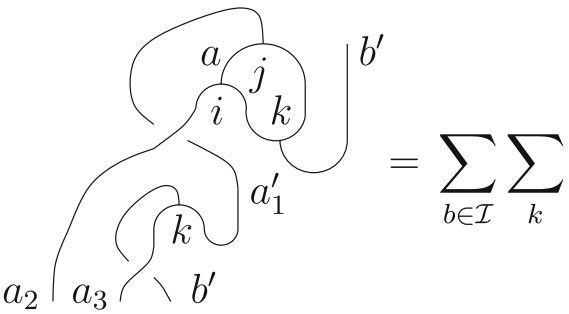

$\mapsto \sum_{b \in \mathcal{I}} \sum_{k}$
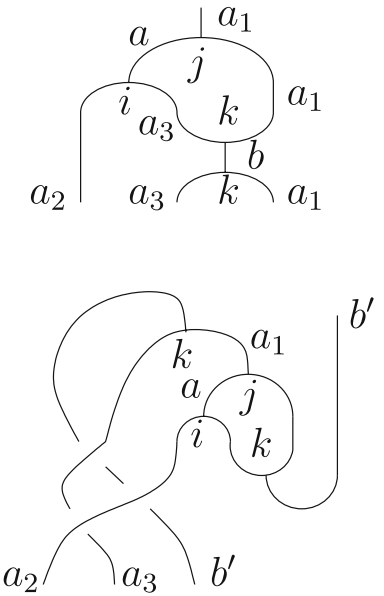

We have introduced $S, \alpha, \beta$ all as isomorphisms on $\oplus_{a, a_{1} \in \mathcal{I}} \mathcal{V}_{a a_{1}}^{a_{1}} \otimes \mathcal{V}_{a_{2} a_{3}}^{a}$. They satisfy the following well-known equation [MSei1,MSei3,H10,HKo3]:

$$
S \alpha=\beta S \text {. }
$$

We proved in [HKo3] that $S$ is determined by the identity (4.34) up to a constant $S_{e}^{e}$. We will solve Eq. (4.34) for $S$ graphically below.

\section{Proposition 4.6.}
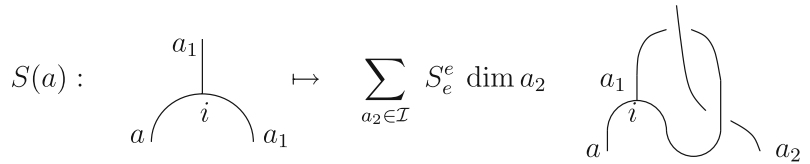

Proof. Since we know that Eq. (4.34) determines $S$ up to an overall constant $S_{e}^{e}$, we only need to check that (4.35) gives a solution to (4.34). 
Combining (4.31) with (4.35), we obtain that

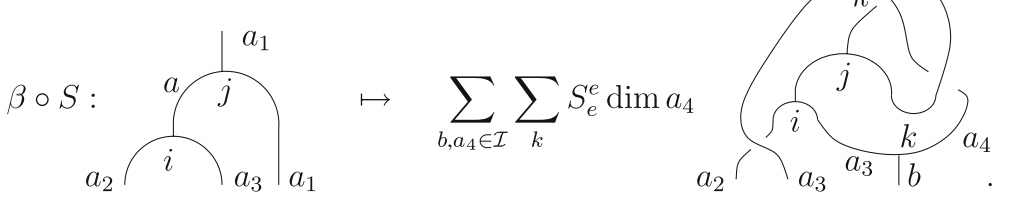

The diagram in the right hand side of (4.36) can be deformed as follows:
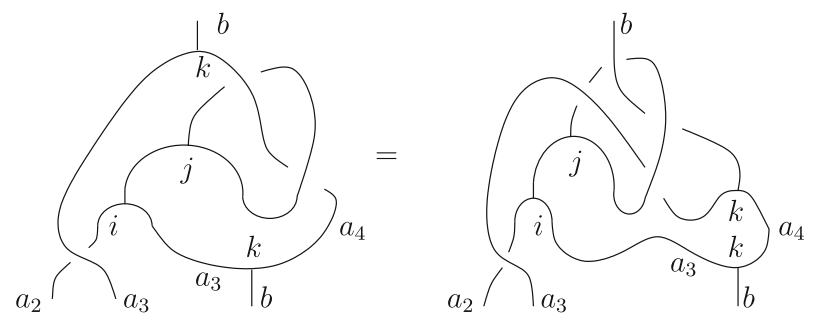

By (4.18), we have

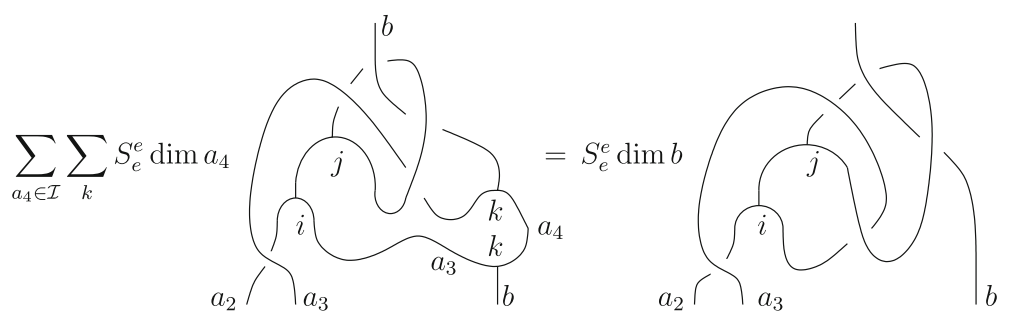

On the other hand, combining (4.29) with (4.35), we obtain<smiles>CCC(CC(C)C)CC(C)C</smiles><smiles>CCC[As]</smiles>



Notice the diagram on the right hand side of (4.39) is exactly a deformation of the diagram on the right hand side of (4.38). Hence we obtain that the map defined as (4.35) gives a solution to the equation $S \alpha=\beta S$.

To determine $S$ completely, we need to determine $S_{e}^{e}$. This can be done by using other identities satisfied by $S$.

\section{Proposition 4.7.}

$$
S^{2}(a)\left(\mathcal{Y}_{a a_{1} ; i}^{a_{1} ;(1)}\right)=\tilde{A}_{0}\left(\mathcal{Y}_{a a_{1} ; i}^{a_{1} ;(1)}\right)
$$


Proof. By the definition of $S$-cation on $\mathcal{Y}_{a a_{1} ; i}^{a_{1} ;(1)} \in \mathcal{V}_{a a_{1}}^{a_{1}}$, we have

$$
\Psi_{1}\left(S^{2}(a)\left(\mathcal{Y}_{a a_{1} ; i}^{a_{1} ;(1)}\right)\right)\left(w_{a} ; z, \tau\right)=\Psi_{1}\left(\mathcal{Y}_{a a_{1} ; i}^{a_{1} ;(1)}\right)\left(\tau^{L(0)}\left(\frac{-1}{\tau}\right)^{L(0)} w_{a} ;-z, \tau\right)
$$

for $w_{a} \in W_{a}$. Keep in mind our convention on the branch cut for a logarithm. We have

$$
\tau^{L(0)}\left(\frac{-1}{\tau}\right)^{L(0)} w_{a}=e^{\pi i L(0)} w_{a}
$$

Hence we obtain

$$
\Psi_{1}\left(S^{2}(a)\left(\mathcal{Y}_{a a_{1} ; i}^{a_{1} ;(1)}\right)\right)\left(w_{a} ; z, \tau\right)=\Psi_{1}\left(\mathcal{Y}_{a a_{1} ; i}^{a_{1} ;(1)}\right)\left(e^{\pi i L(0)} w_{a} ;-z, \tau\right) .
$$

By (A.47), we also have

$$
\begin{aligned}
& \Psi_{1}\left(\mathcal{Y}_{a a_{1} ; i}^{a_{1} ;(1)}\right)\left(e^{\pi i L(0)} w_{a} ;-z, \tau\right) \\
& =E\left(\operatorname{Tr}_{W_{a_{1}}} \mathcal{Y}_{a a_{1} ; i}^{a_{1} ;(1)}\left(\mathcal{U}\left(e^{-2 \pi i z}\right) e^{\pi i L(0)} w_{a}, e^{-2 \pi i z}\right) q_{\tau}^{L(0)-\frac{c}{24}}\right) \\
& =E\left(\operatorname{Tr}_{\left(W_{a_{1}}\right)}, \tilde{A}_{0}\left(\mathcal{Y}_{a a_{1} ; i}^{a_{1} ;(1)}\right)\left(e^{\pi i L(0)} \mathcal{U}\left(e^{2 \pi i z}\right) w_{a}, e^{\pi i} e^{2 \pi i z}\right) q_{\tau}^{L(0)-\frac{c}{24}}\right) \\
& \left.=E\left(\operatorname{Tr}_{\left(W_{a_{1}}\right)}\right)^{\prime} \tilde{A}_{0}\left(\mathcal{Y}_{a a_{1} ; i}^{a_{1} ;(1)}\right)\left(\mathcal{U}\left(e^{2 \pi i z}\right) w_{a}, e^{2 \pi i z}\right) q_{\tau}^{L(0)-\frac{c}{24}}\right) \\
& =\Psi_{1}\left(\tilde{A}_{0}\left(\mathcal{Y}_{a a_{1} ; i}^{a_{1} ;(1)}\right)\right)\left(w_{a} ; z, \tau\right) \text {. }
\end{aligned}
$$

Therefore, combining (4.42) and (4.43), we obtain (4.40).

The following lemma is proved in [BK2].

Lemma 4.8. Let $D^{2}=\sum_{a \in \mathcal{I}} \operatorname{dim}^{2} a$. Then $D \neq 0$ and we have

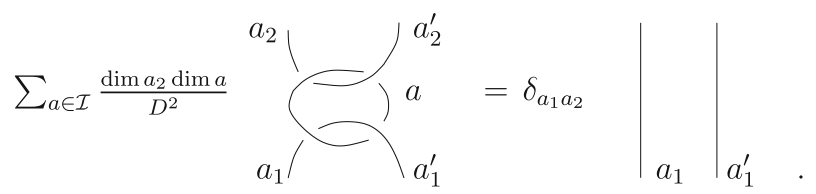

\section{Proposition 4.9.}

$$
\left(S_{e}^{e}\right)^{2}=\frac{1}{D^{2}}
$$

Proof. By (4.35), we have

$$
S^{2}(a): \overbrace{i}^{a_{1}} \mapsto \sum_{a_{2}, a_{3} \in \mathcal{I}}\left(S_{e}^{e}\right)^{2} \operatorname{dim} a_{2} \operatorname{dim} a_{3}
$$

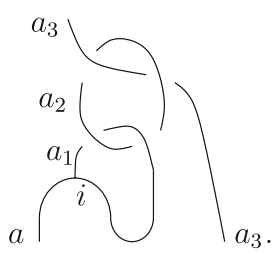


Apply (4.12) to the graph in the right hand side of (4.46), we obtain

$$
\sum_{a_{2} \in \mathcal{I}} \frac{\operatorname{dim} a_{2} \operatorname{dim} a_{3}}{D^{2}}
$$

By (4.44), the right-hand side of (4.47) equals

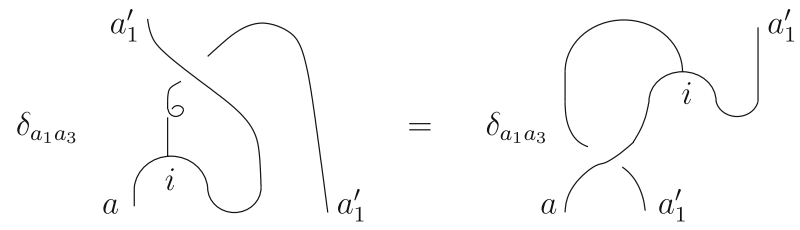

By (4.40) and (4.22), we obtain (4.45).

So far, we have determined $S_{e}^{e}$ up to a sign. Now we consider the relation between $S$ and another generator of the modular group $T: \tau \mapsto \tau+1$. We define a $T$-action on $\Psi_{1}\left(\mathcal{Y}_{a a_{1} ; i}^{a_{1}}\right)$ as follows:

$$
T\left(\Psi_{1}\left(\mathcal{Y}_{a a_{1} ; i}^{a_{1}}\right)\right)\left(w_{a} ; z, \tau\right):=\Psi_{1}\left(\mathcal{Y}_{a a_{1} ; i}^{a_{1}}\right)\left(w_{a} ; z, \tau+1\right) .
$$

It is clear that this action induces an action of $T$ on $\mathcal{V}_{a a_{1}}^{a_{1}}$ for all $a, a_{1} \in \mathcal{I}$, given by

$$
\left.T\right|_{\mathcal{V}_{a a_{1}}^{a_{1}}}=e^{2 \pi i\left(h_{a}-\frac{c}{24}\right)},
$$

where $h_{a}$ is the lowest conformal weight of $W_{a}$.

Lemma 4.10. $S$ and $T$ satisfy the following relation:

$$
\left(T^{-1} S\right)^{3}=S^{2}=T^{-1} S^{2} T .
$$

Proof. Let $w_{a} \in W_{a}$. We have

$$
\begin{aligned}
& \left(T^{-1} S\right)^{3}\left(\left(\Psi_{1}\left(\mathcal{Y}_{a a_{1} ; i}^{a_{1} ;(1)}\right)\right)\left(w_{a} ; z, \tau\right)\right. \\
& =\Psi_{1}\left(\mathcal{Y}_{a a_{1} ; i}^{a_{1} ;(1)}\right)\left(\tau^{L(0)}\left(\frac{-1}{\frac{-1}{\tau-1}-1}\right)^{L(0)}\left(\frac{-1}{\tau-1}\right)^{L(0)} w_{a},-z, \tau\right) .
\end{aligned}
$$

Keeping in mind our choice of branch cut, then it is easy to show that

$$
\tau^{L(0)}\left(\frac{-1}{\frac{-1}{\tau-1}-1}\right)^{L(0)}\left(\frac{-1}{\tau-1}\right)^{L(0)} w_{a}=e^{\pi i L(0)} w_{a} .
$$

By (4.42), we obtain the first equality of (4.51). The proof of the second equality (4.51) is similar. 
Proposition 4.11. Let $p_{ \pm}=\sum_{a \in \mathcal{I}} e^{ \pm 2 \pi i h_{a}} \operatorname{dim}^{2} a$. Then we have

$$
S_{e}^{e}=\frac{1}{p_{-}} e^{2 \pi i c / 8}=\frac{1}{p_{+}} e^{-2 \pi i c / 8} .
$$

Proof. In the proof of Theorem 3.1.16 in [BK2], Bakalov and Kirillov proved an identity, which, in our own notation, can be written as follows:

$$
\frac{1}{\left(S_{e}^{e}\right)^{2} D^{2}} e^{-2 \pi i \frac{c}{24}} S T^{-1} S=\frac{1}{S_{e}^{e}} \frac{p_{+}}{D^{2}} e^{2 \pi i \frac{2 c}{24}} T S T .
$$

By (4.51) and the fact $p_{-} p_{+}=D^{2}$ which is proved in [BK2], we simply obtain that

$$
S_{e}^{e}=\frac{1}{p_{-}} e^{2 \pi i c / 8} .
$$

Using (4.45) and $p_{+} p_{-}=D^{2}$, we also obtain the second equality.

We thus define

$$
D:=p_{-} e^{-2 \pi i c / 8}=p_{+} e^{2 \pi i c / 8} .
$$

Notice that this notation is compatible with the definition of $D^{2}$. Then the action of modular transformation $S(a)$ on $\oplus_{a_{1} \in \mathcal{I}} \operatorname{Hom}_{V}\left(W_{a} \otimes W_{a_{1}}, W_{a_{1}}\right)$ can be expressed graphically as follows:

$$
S(a): \quad \overbrace{i}^{a_{1}} \mapsto \sum_{a_{1} \in \mathcal{I}} \frac{\operatorname{dim} a_{2}}{D} a_{a}^{a_{1}}
$$

Proposition 4.12.

$$
S^{-1}(a): \overbrace{i}^{a_{1}} \mapsto \sum_{a_{2} \in \mathcal{I}} \frac{\operatorname{dim} a_{2}}{D}
$$

Proof. Composing the map (4.55) with (4.56), we obtain a map given as follows:



Apply (4.44) to the graph in (4.57), it is easy to see that the above map is the identity map.

Remark 4.13. Bakalov and Kirillov obtained the same formula (4.55) in [BK2] by directly working with the modular tensor category and solving equations obtained in the so-called Lego-Teichmüller game [BK1]. In our approach, we see the direct link between the modular transformations of $q$-traces of the product (or iterate) of intertwining operators and their graphic representations in a modular tensor category. 


\section{Proposition 4.14.}

$$
(S(a))^{*}: a \bigcup_{j}^{a_{1}} \mapsto \sum_{a_{2} \in \mathcal{I}} \frac{\operatorname{dim} a_{2}}{D}
$$

Proof. We only prove (4.58). The proof of (4.59) is analogous to that of (4.58).

It is enough to show that the pairing between the image of (4.35) and that of (4.58) still gives $\delta_{i j}$. This can be proved as follows:

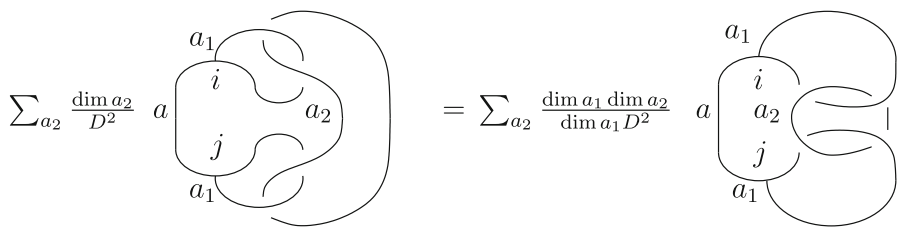

By (4.44), the right hand side of (4.60) equals

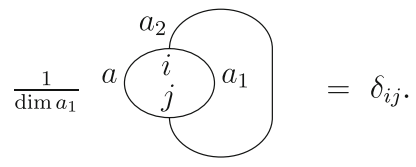

\section{Categorical Formulations and Constructions}

In this section, we give a categorical formulation of modular invariant conformal full field algebra over $V^{L} \otimes V^{L}$, open-string vertex operator algebra over $V$ equipped with nondegenerate invariant bilinear forms and Cardy condition. Then we introduce a notion called Cardy $\mathcal{C}_{V} \mid \mathcal{C}_{V \otimes V}$-algebra. In the end, we give a categorical construction of such an algebra in the Cardy case [FFFS2].

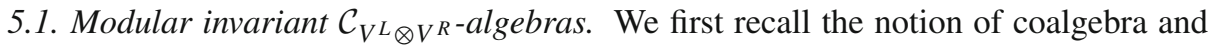
Frobenius algebra ([FS]) in a tensor category.

Definition 5.1. A coalgebra $A$ in a tensor category $\mathcal{C}$ is an object with a coproduct $\Delta \in \operatorname{Mor}(A, A \otimes A)$ and a counit $\epsilon \in \operatorname{Mor}\left(A, \mathbf{1}_{\mathcal{C}}\right)$ such that

$$
\left(\Delta \otimes \mathrm{id}_{A}\right) \circ \Delta=\left(\mathrm{id}_{A} \otimes \Delta\right) \circ \Delta, \quad\left(\epsilon \otimes \mathrm{id}_{A}\right) \circ \Delta=\mathrm{id}_{A}=\left(\operatorname{id}_{A} \otimes \epsilon\right) \circ \Delta,
$$

which can also be expressed in term of the following graphic equations:
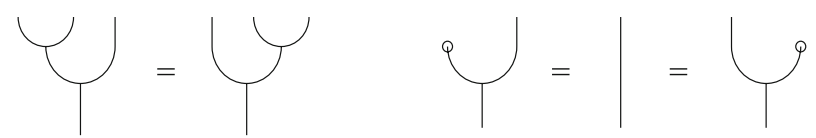
Definition 5.2. Frobenius algebra in $\mathcal{C}$ is an object that is both an algebra and a coalgebra and for which the product and coproduct are related by

$$
\left(\mathrm{id}_{A} \otimes m\right) \circ\left(\Delta \otimes \mathrm{id}_{A}\right)=\Delta \circ m=\left(m \otimes \mathrm{id}_{A}\right) \circ\left(\mathrm{id}_{A} \otimes \Delta\right),
$$

or as the following graphic equations:
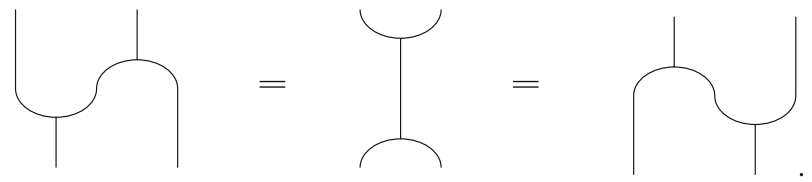

A Frobenius algebra is called symmetric if the following condition is satisfied:
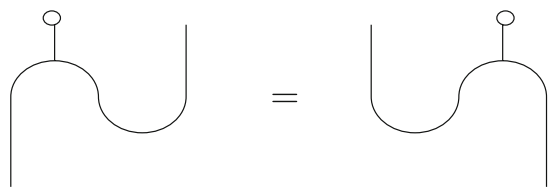

Let $V^{L}$ and $V^{R}$ be vertex operator algebras satisfying the conditions in Theorem 0.1 . Then the vertex operator algebra $V^{L} \otimes V^{R}$ also satisfies the conditions in Theorem 0.1 [HKo1]. Thus $\mathcal{C}_{V^{L} \otimes V^{R}}$ also has a structure of modular tensor category. In particular, we choose the braiding structure on $\mathcal{C}_{V^{L} \otimes V^{R}}$ to be $\mathcal{R}_{+-}$which is defined in [Ko2]. The twist $\theta_{+-}$for each $V^{L} \otimes V^{R}$-module is defined by

$$
\theta_{+-}=e^{-2 \pi i L^{L}(0)} \otimes e^{2 \pi i L^{R}(0)}
$$

Duality maps are naturally induced from those of $\mathcal{C}_{V^{L}}$ and $\mathcal{C}_{V^{R}}$.

The following theorem is proved in [Ko1].

Theorem 5.3. The category of conformal full field algebras over $V^{L} \otimes V^{R}$ equipped with nondegenerate invariant bilinear forms is isomorphic to the category of commutative Frobenius algebra in $\mathcal{C}_{V^{L} \otimes V^{R}}$ with a trivial twist.

Remark 5.4. In a ribbon category, it was proved in [FFRS] that a commutative Frobenius algebra with a trivial twist is equivalent to a commutative symmetric Frobenius algebra.

Let $\mathcal{I}^{L}$ and $\mathcal{I}^{R}$ denote the set of equivalent class of irreducible $V^{L}$-modules and $V^{R}$-modules respectively. We use $a$ and $a_{i}$ for $i \in \mathbb{N}$ to denote elements in $\mathcal{I}^{L}$ and we use $e$ to denote the equivalent class of $V^{L}$. We use $\bar{a}$ and $\bar{a}_{i}$ for $i \in \mathbb{N}$ to denote elements in $\mathcal{I}^{R}$, and $\bar{e}$ to denote the equivalent class of $V^{R}$. For each $a \in \mathcal{I}^{L}\left(\bar{a} \in \mathcal{I}^{R}\right)$, we choose a representative $W_{a}\left(W_{\bar{a}}\right)$. We denote the vector space of intertwining operators of type $\left(\begin{array}{c}W_{a_{3}} \\ W_{a_{1} W_{a_{2}}}\end{array}\right)$ and $\left(\begin{array}{c}W_{\bar{a}_{3}} \\ W_{\bar{a}_{1} W_{\bar{a}_{2}}}\end{array}\right)$ as $\mathcal{V}_{a_{1} a_{2}}^{a_{3}}$ and $\overline{\mathcal{V}}_{\bar{a}_{1} \bar{a}_{2}}^{\bar{a}_{3}}$ respectively, the fusion rule as $N_{a_{1} a_{2}}^{a_{3}}$ and $N_{\bar{a}_{1} \bar{a}_{2}}^{\bar{a}_{3}}$ respectively.

A conformal full field algebra over $V^{L} \otimes V^{R}$, denoted as $A_{c l}$, is a direct sum of irreducible modules of $V^{L} \otimes V^{R}$, i.e.

$$
A_{c l}=\oplus_{\alpha=1}^{N} W_{r(\alpha)} \otimes W_{\bar{r}(\alpha)},
$$


where $r:\{1, \ldots, N\} \rightarrow \mathcal{I}^{L}$ and $\bar{r}:\{1, \ldots, N\} \rightarrow \mathcal{I}^{R}$, for some $N \in \mathbb{Z}_{+}$. Let $\left\{e_{a b ; i}^{c}\right\}$ and $\left\{\bar{e}_{\bar{a} \bar{b} ; j}^{\bar{c}}\right\}$ be basis for $\mathcal{V}_{a b}^{c}$ and $\overline{\mathcal{V}}_{\bar{a} \bar{b}}^{\bar{c}}$, and $\left\{f_{c ; i}^{a b}\right\}$ and $\left\{\bar{f}_{\bar{c} ; j}^{\bar{a} \bar{b}}\right\}$ be the dual basis respectively. Then the vertex operator $\mathbb{Y}$ can also be expanded as follows:

$$
\mathbb{Y}=\sum_{\alpha, \beta, \gamma} \sum_{i, j} d_{\alpha \beta}^{\gamma}\left(f_{r(\gamma) ; i}^{r(\alpha) r(\beta)}, \bar{f}_{\bar{r}(\gamma) ; j}^{\bar{r}(\alpha) \bar{r}(\beta)}\right) e_{r(\alpha) r(\beta)}^{r(\gamma) ; i} \otimes \bar{e}_{\bar{r}(\alpha) ; \bar{r}(\beta)}^{\bar{r}(\gamma),},
$$

where $d_{\alpha \beta}^{\gamma}$ defines a bilinear map

$$
\left(\mathcal{V}_{r(\alpha) r(\beta)}^{r(\gamma)}\right)^{*} \otimes\left(\overline{\mathcal{V}}_{\bar{r}(\alpha) \bar{r}(\beta)}^{\bar{r}(\gamma)}\right)^{*} \rightarrow \mathbb{C}
$$

for all $\alpha, \beta, \gamma=1, \ldots, N$ for some $N \in \mathbb{N}$.

Since the trace function picks out $\gamma=\beta$ terms, we define $\mathbb{Y}_{\alpha}^{\text {diag }}$ by

$$
\mathbb{Y}_{\alpha}^{\text {diag }}:=\sum_{\beta} \sum_{i, j} d_{\alpha \beta}^{\beta}\left(f_{r(\beta) ; i}^{r(\alpha) r(\beta)}, \bar{f}_{\bar{r}(\beta) ; j}^{\bar{r}(\alpha) \bar{r}(\beta)}\right) e_{r(\alpha) r(\beta)}^{r(\beta) ; i} \otimes \bar{e}_{\bar{r}(\alpha) ; \bar{r}(\beta)}^{\bar{r}(\beta) ; j} .
$$

Let $\mathbb{Y}^{\text {diag }}:=\sum_{\alpha} \mathbb{Y}_{\alpha}^{\text {diag }}$. Of course, it is obvious to see that such defined $\mathbb{Y}^{\text {diag }}$ is independent of the choice of basis. We denote the representation of the modular transformation $S: \tau \mapsto \frac{-1}{\tau}$ on $\oplus_{b \in \mathcal{I}^{L}} \mathcal{V}_{a b}^{b}$ and $\oplus_{\bar{b} \in \mathcal{I}^{R}} \mathcal{V}_{\bar{a} \bar{b}}^{\bar{b}}$, by $S^{L}(a)$ and $S^{R}(\bar{a})$ respectively.

In [HKo3], we defined the notion of modular invariant conformal full field algebra over $V^{L} \otimes V^{R}$ (see [HKo3] for the precise definition). It basically means that the $n$-point genus-one correlation functions built out of $q-\bar{q}$-traces are invariant under the action of modular group $S L(2, \mathbb{Z})$ for all $n \in \mathbb{N}$. Moreover, we proved the following results in [HKo3].

Proposition 5.5. $A_{c l}$, a conformal full field algebra over $V^{L} \otimes V^{R}$, is modular invariant if it satisfies $c^{L}-c^{R}=0 \bmod 24$ and

$$
S^{L}(r(\alpha)) \otimes\left(S^{R}(\bar{r}(\alpha))\right)^{-1} \mathbb{Y}_{\alpha}^{\text {diag }}=\mathbb{Y}_{\alpha}^{\text {diag }}
$$

for all $\alpha=1, \ldots, N$.

We denote the morphism in

$$
\operatorname{Hom}_{C_{V} L_{\otimes V} R}\left(\left(W_{r(\alpha)} \otimes W_{\bar{r}(\alpha)}\right) \otimes\left(W_{r(\beta)} \otimes W_{\bar{r}(\beta)}\right), W_{r(\beta)} \otimes W_{\bar{r}(\beta)}\right)
$$

which corresponds to $\mathbb{Y}_{\alpha}^{\text {diag }}$ by $m_{\mathbb{Y}_{\alpha}^{\text {diag }}}$. Then (5.9) is equivalent to the following categorical condition:

$$
S^{L}(r(\alpha)) \otimes\left(S^{R}(\bar{r}(\alpha))\right)^{-1} m_{\mathbb{Y}_{\alpha}^{\text {diag }}}=m_{\mathbb{Y}_{\alpha}^{\text {diag }}}
$$

for all $\alpha=1, \ldots, N$.

Now we choose a basis $\left\{b_{a \otimes \bar{a} ; i}^{A_{c l}}\right\}$ of $\operatorname{Hom}_{C_{V} L_{\otimes V}}\left(W_{a} \otimes W_{\bar{a}}, A_{c l}\right)$ and a basis $\left\{b_{A_{c l}}^{a \otimes \bar{a} ; i}\right\}$ of $\operatorname{Hom}_{C_{V} L_{\otimes V}}\left(A_{c l}, W_{a} \otimes W_{\bar{a}}\right)$ as follows:

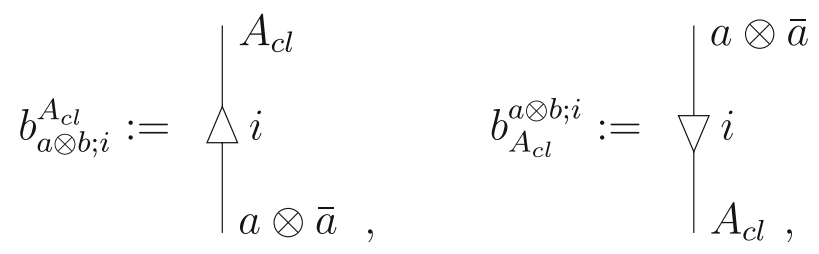


satisfying the following conditions: for all $a \in \mathcal{I}^{L}, \bar{a} \in \mathcal{I}^{R}$,

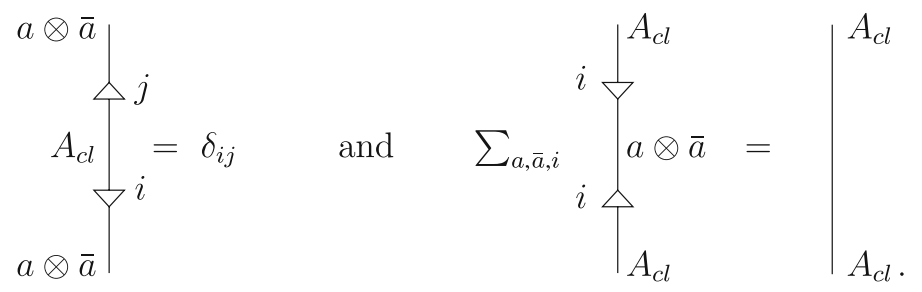

Then the condition (5.10) can be further expressed graphically as follows:

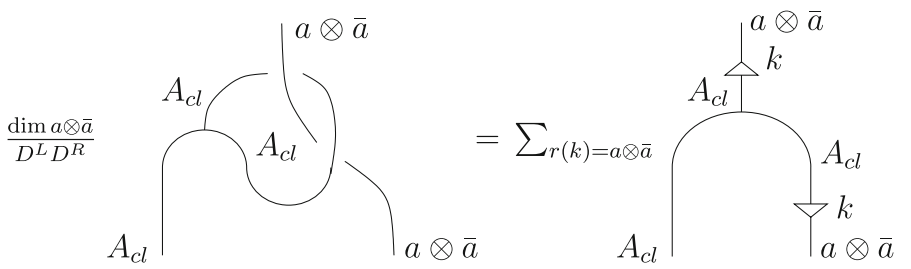

for all $a \in \mathcal{I}^{L}, \bar{a} \in \mathcal{I}^{R}$, where $D^{L}$ and $D^{R}$ are the $D$ defined by (4.54) in $\mathcal{C}_{V^{L}}$ and $\mathcal{C}_{V^{R}}$ respectively.

Definition 5.6. Let $V^{L}$ and $V^{R}$ be so that $c^{L}-c^{R}=0 \bmod 24$. A modular invariant

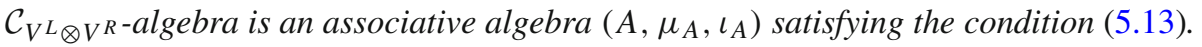

Some properties of the modular invariant algebra follow immediately from the above definition, such as the following famous condition [MSei3]:

$$
\sum_{a \in \mathcal{I}^{L}, \bar{a} \in \mathcal{I}^{R}}\left(S^{L}(e)\right)_{a b} N_{b \bar{b}}\left(S^{R}(\bar{e})\right)_{\bar{b} \bar{a}}^{-1}=N_{a \bar{a}}
$$

where $N_{a \bar{a}}$ is the multiplicity of $W_{a} \otimes W_{\bar{a}}$ in $A_{c l}$ for $a \in \mathcal{I}^{L}, \bar{a} \in \mathcal{I}^{R}$. We leave a more systematic study of modular invariant $\mathcal{C}_{V^{L} \otimes V^{R}}$-algebras to elsewhere.

Theorem 5.7. Let $V^{L}$ and $V^{R}$ be so that $c^{L}-c^{R}=0 \bmod 24$. The following two notions are equivalent:

1. modular invariant conformal full field algebra over $V^{L} \otimes V^{R}$ equipped with a nondegenerate invariant bilinear form,

2. modular invariant commutative Frobenius algebra with a trivial twist.

Proof. The theorem follows from Theorem 5.3 and the equivalence between (5.9) and (5.13) immediately. 
Remark 5.8. In the case $V^{L} \cong \mathbb{C} \cong V^{R}$, a modular invariant commutative Frobenius algebra with a trivial twist in $\mathcal{C}_{V^{L} \otimes V^{R}}$ is simply a commutative Frobenius algebra over $\mathbb{C}$, which is equivalent to a 2-dimensional topological field theory (see for example [BK2]). In this case, the modular invariance condition holds automatically.

5.2. $\operatorname{Cardy} \mathcal{C}_{V} \mid \mathcal{C}_{V \otimes V}$-algebras. For an open-string vertex operator algebra $V_{\text {op }}$ over $V$ equipped with a nondegenerate invariant bilinear form $(\cdot, \cdot)_{o p}$, there is an isomorphism $\varphi_{o p}: V_{o p} \rightarrow V_{o p}$ induced from $(\cdot, \cdot)_{o p}$ (recall (3.40)).

In this case, $V_{o p}$ is a $V$-module and $Y_{o p}^{f}$ is an intertwining operator. By comparing (1.26) with (3.37), and (1.27) with (3.38), we see that the conditions (1.26) and (1.27) can be rewritten as

$$
\begin{aligned}
Y_{o p}^{f} & =\varphi_{o p}^{-1} \circ \sigma_{123}\left(Y_{o p}^{f}\right) \circ\left(\varphi_{o p} \otimes \operatorname{id}_{V_{o p}}\right) \\
& =\varphi_{o p}^{-1} \circ \sigma_{132}\left(Y_{o p}^{f}\right) \circ\left(\operatorname{id}_{o p} \otimes \varphi_{o p}\right) .
\end{aligned}
$$

Remark 5.9. The representation theory of the open-string vertex operator algebra can be developed. In that context, $\sigma_{123}\left(Y_{o p}^{f}\right)$ gives $V_{o p}^{\prime}$ a right $V_{o p}$-module structure and Eq. (5.15) is equivalent to the statement that $\varphi_{o p}$ is an isomorphism between two right $V_{o p}$-modules. Similarly, $\sigma_{132}\left(Y_{o p}^{f}\right)$ gives $V_{o p}^{\prime}$ a left $V_{o p}$-module structure and Eq. (5.16) is equivalent to the statement that $\varphi_{o p}$ is an isomorphism between two left $V_{o p}$-modules. But we do not need it in this work.

Theorem 5.10. The category of open-string vertex operator algebras over $V$ equipped with a nondegenerate invariant bilinear form is isomorphic to the category of symmetric Frobenius algebras in $\mathcal{C}_{V}$.

Proof. We have already shown in [HKo1] that an open-string vertex operator algebra over $V$ is equivalent to an associative algebra in $\mathcal{C}_{V}$.

Let $V_{o p}$ be an open-string vertex operator algebra over $V$. Giving a nondegenerate invariant bilinear form (recall (1.26) and (1.27)) on $V_{o p}$ is equivalent to giving an isomorphism $\varphi_{o p}: V_{o p} \rightarrow V_{o p}^{\prime}$ satisfying the conditions (5.15) and (5.16). If we define




then (5.15) and (5.16) can be rewritten as
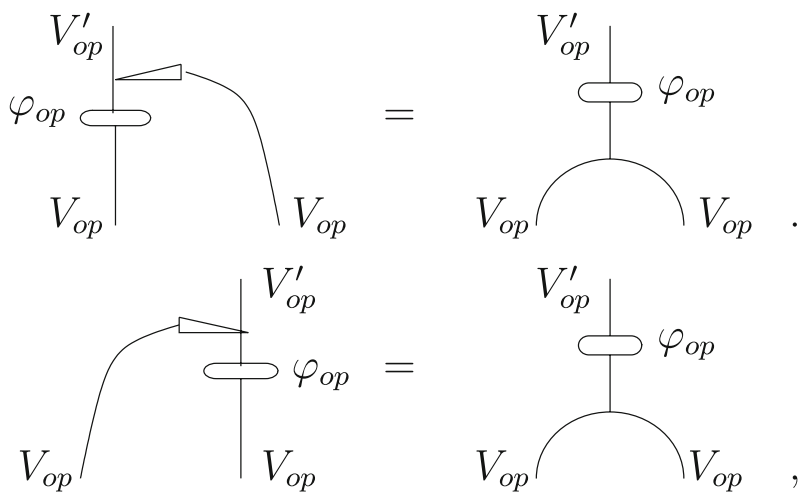

Using the map $\varphi_{o p}$ and its inverse, we can obtain a natural coalgebra structure on $F$ defined as follows:

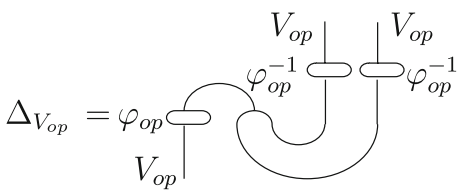

$$
\epsilon_{V_{o p}}=\overbrace{V_{o p}}^{0} \varphi_{o p}
$$

Similar to the proof of [Ko1, thm.4.15], (5.19) and (5.20) imply that such defined $\Delta_{V_{o p}}$ and $\epsilon_{V_{o p}}$ give $V_{o p}$ a Frobenius algebra structure. Moreover, we also showed in [Ko1] that (5.19) implies the equality between $\varphi_{o p}$ and the left hand side of (5.4). Similarly, using (5.20), we can show that the right hand side of (5.4) also equals $\varphi_{o p}$. Thus $V_{o p}$ has a structure of symmetric Frobenius algebra.

We thus obtain a functor from the first category to the second category.

Conversely, given a symmetric Frobenius algebra in $\mathcal{C}_{V}$, in [HKo1], we showed that it gives an open-string vertex operator algebra over $V$. It is shown in [FRS2] that either side of (5.4) is an isomorphism. Take $\varphi_{o p}$ to be either side of (5.4). Then (5.19) and (5.20) follow automatically from the definition (5.17) and (5.18). They are nothing but the invariance properties (recall $(5.15)(5.16)$ ) of the bilinear form associated with $\varphi_{o p}$. Thus we obtain a functor from the second category to the first category.

It is routine to check that these two functors are inverse to each other.

Now we consider an open-closed field algebra over $V$ given in (2.12) equipped with nondegenerate invariant bilinear forms $(\cdot, \cdot)_{o p}$ and $(\cdot, \cdot)_{c l}$. We assume that $V_{c l}$ and $V_{o p}$ have the following decompositions:

$$
V_{c l}=\oplus_{i=1}^{N_{c l}} W_{r_{L}(i)} \otimes W_{r_{R}(i)}, \quad V_{o p}=\oplus_{i=1}^{N_{o p}} W_{r(i)},
$$

where $r_{L}, r_{R}:\left\{1, \ldots, N_{c l}\right\} \rightarrow \mathcal{I}$ and $r:\left\{1, \ldots, N_{o p}\right\} \rightarrow \mathcal{I}$. We denote the embedding $b_{(i)}^{V_{o p}}: W_{r(i)} \hookrightarrow V_{o p}$, the projection $b_{V_{o p}}^{(i)}: V_{o p} \rightarrow W_{r(i)}$ and $b_{(i)}^{V_{c l}}: W_{r_{L}(i)} \otimes W_{r_{R}(i)} \hookrightarrow$ 
$T\left(V_{c l}\right)$ by the following graphs:

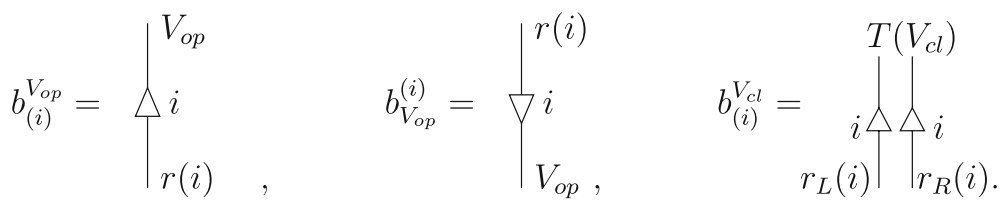

We denote the map $\iota_{c l-o p}: T\left(V_{c l}\right) \rightarrow V_{o p}[\mathrm{Ko} 2]$ by the following graph:

$$
\iota_{c l-o p}:=\overbrace{T\left(V_{c l}\right)}^{V_{o p}} .
$$

Now we can express the Cardy condition (3.73) in graphs. The left hand side of (3.73) can be expressed by:

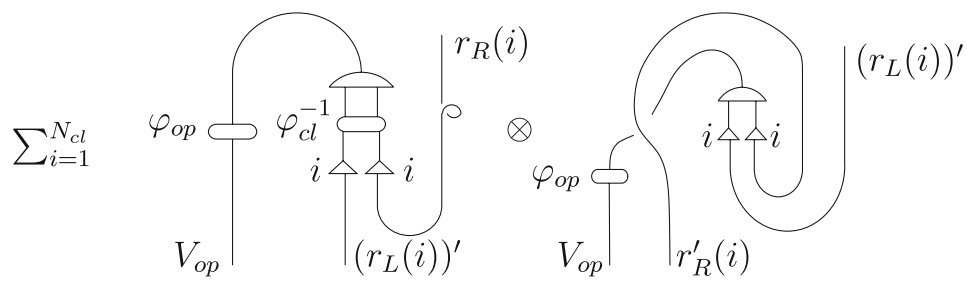

By the universal property of tensor product, for $a_{i} \in \mathcal{I}, i=1, \ldots, 6$, we have a canonical isomorphism:

$$
\begin{aligned}
\oplus_{a \in \mathcal{I}} \mathcal{V}_{a_{1} a}^{a_{4}} \otimes \mathcal{V}_{a_{2} a_{3}}^{a} \stackrel{\cong}{\rightarrow} \operatorname{Hom}_{V}\left(W_{a_{1}} \otimes\left(W_{a_{2}} \otimes W_{a_{3}}\right), W_{a_{4}}\right) \\
\mathcal{Y}_{1} \otimes \mathcal{Y}_{2} \mapsto m_{\mathcal{Y}_{1}} \circ\left(\operatorname{id}_{W_{a_{1}}} \otimes m_{\mathcal{Y}_{2}}\right)
\end{aligned}
$$

Under this canonical isomorphism, the Cardy condition (3.73) can be viewed as a condition on two morphisms in $\operatorname{Hom}_{V}\left(V_{o p} \otimes\left(V_{o p} \otimes W_{r_{R}(i)}\right), W_{r_{R}(i)}\right)$. In particular, the left hand side of (3.73) viewed as a morphism in $\operatorname{Hom}_{V}\left(V_{o p} \otimes\left(V_{o p} \otimes W_{r_{R}(i)}\right), W_{r_{R}(i)}\right)$ can be expressed as follows:

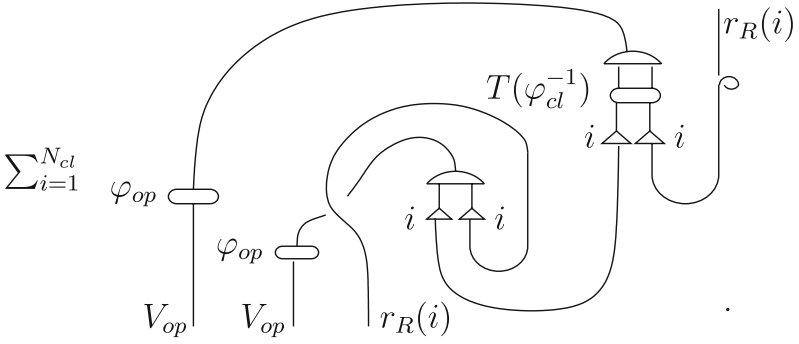


We define a morphism $\iota_{c l-o p}^{*}: V_{o p} \rightarrow T\left(V_{c l}\right)$ by

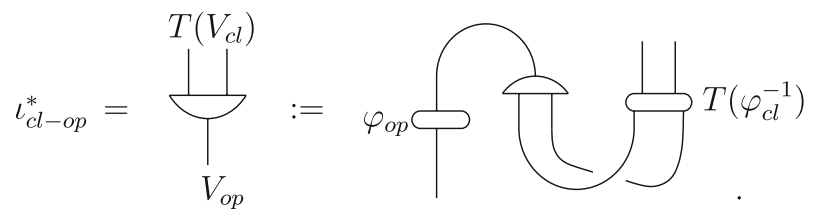

Then using the morphism $\iota_{c l-o p}^{*}$, we can rewrite the graph in (5.26) as follows:


Using (4.55), (5.26) and (5.28), we obtain a graphic version of the Cardy condition (3.73) as follows:

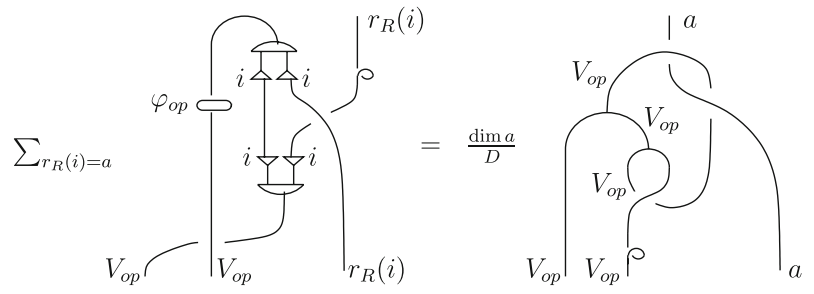

Using the Frobenius properties of $V_{o p}$, one can show that (5.29) is equivalent to the following condition:

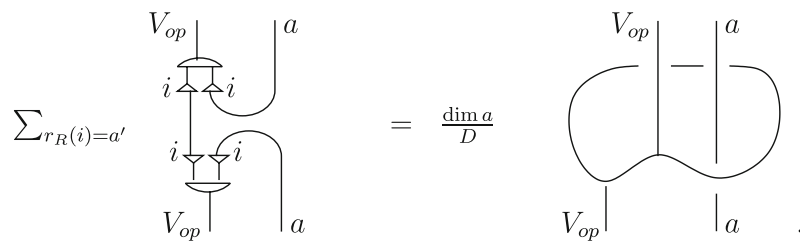

The asymmetry between chiral and antichiral parts in (5.29)(5.30) is superficial. Using (4.44), one can show that (5.30) is further equivalent to the following condition:

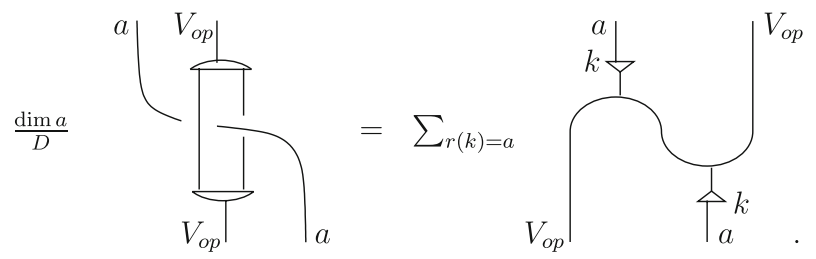

We recall a definition in [Ko2]. 
Definition 5.11. An open-closed $\mathcal{C}_{V} \mid \mathcal{C}_{V \otimes V}$-algebra, denoted as $\left(A_{o p} \mid A_{c l}, \iota_{c l-o p}\right)$, consists of a commutative symmetric associative algebra $A_{c l}$ in $\mathcal{C}_{V \otimes V}$, an associative algebra $A_{o p}$ in $\mathcal{C}_{V}$ and an associative algebra morphism $\iota_{c l-o p}: T\left(V_{c l}\right) \rightarrow V_{o p}$, satisfying the following condition:

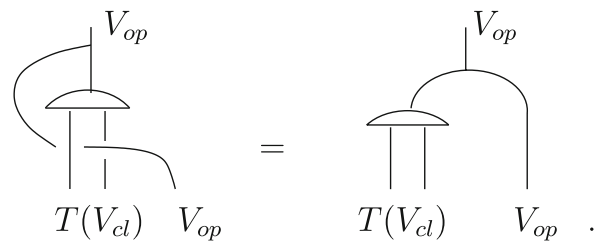

The following theorem is proved in [Ko2].

Theorem 5.12. The category of open-closed field algebras over $V$ is isomorphic to the category of open-closed $\mathcal{C}_{V} \mid \mathcal{C}_{V \otimes V}$-algebras.

Definition 5.13. $A$ Cardy $\mathcal{C}_{V} \mid \mathcal{C}_{V \otimes V}$-algebra is an open-closed $\mathcal{C}_{V} \mid \mathcal{C}_{V \otimes V}$-algebra $\left(A_{o p} \mid A_{c l}, \iota_{c l-o p}\right)$ such that $A_{c l}$ is modular invariant commutative symmetric Frobenius algebra in $\mathcal{C}_{V \otimes V}$ and $A_{\text {op }}$ a symmetric Frobenius algebra in $\mathcal{C}_{V}$ and the Cardy condition (5.30) or (5.31) hold.

Remark 5.14. Notice that, in the case $V=\mathbb{C}$, the Cardy $\mathcal{C}_{V} \mid \mathcal{C}_{V \otimes V}$-algebra (using (5.31)) exactly coincides with the usual algebraic formulation of 2-dimensional open-closed topological field theory [La,Mo1, Mo2, Se2, MSeg, AN,LP]. As we discussed in the introduction, we believe that open-closed partial conformal field theories of all genus satisfying the $V$-invariant boundary condition [Ko2] are classified by Cardy $\mathcal{C}_{V} \mid \mathcal{C}_{V \otimes V^{-}}$ algebras.

The following result is clear.

Theorem 5.15. The category of open-closed field algebras over $V$ equipped with nondegenerate invariant bilinear forms and satisfying the modular invariance condition and the Cardy condition is isomorphic to the category of Cardy $\mathcal{C}_{V} \mid \mathcal{C}_{V \otimes V}$-algebras.

Definition 5.16. $A V$-invariant D-brane associated to a closed algebra $A_{c l}$ in $\mathcal{C}_{V \otimes V}$ is a pair $\left(A_{o p}, \iota_{c l-o p}\right)$ such that the triple $\left(A_{o p} \mid A_{c l}, \iota_{c l-o p}\right)$ gives a Cardy $\mathcal{C}_{V} \mid \mathcal{C}_{V \otimes V^{-}}$ algebra.

D-branes usually form a category as we will see in an example in the next subsection.

5.3. Constructions. In this section, we give a categorical construction of Cardy $\mathcal{C}_{V} \mid \mathcal{C}_{V \otimes V}$-algebra. This construction is called the Cardy case in the physics literature [FFFS2].

Let us first recall the diagonal construction of the close algebra $V_{c l}$ [FFRS,HKo2, Ko1,HKo3]. We will follow the categorical construction given in [Ko1].

Let $V_{c l}$ be the object in $\mathcal{C}_{V \otimes V}$ given as follows:

$$
V_{c l}=\oplus_{a \in \mathcal{I}} W_{a} \otimes W_{a}^{\prime} .
$$

The decomposition of $V_{c l}$ as a direct sum gives a natural embedding $V \otimes V \hookrightarrow V_{c l}$. We denote this embedding as $\iota_{c l}$. We define a morphism $\mu_{c l} \in \operatorname{Hom}_{V \otimes V}\left(V_{c l} \otimes V_{c l}, V_{c l}\right)$ by 


$$
\mu_{c l}=\sum_{a_{1}, a_{2}, a_{3} \in \mathcal{A}} \sum_{i, j=1}^{N_{a_{1} a_{2}}^{a_{3}}}\left\langle f_{a_{3} ; i}^{a_{1} a_{2}}, f_{a_{3}^{\prime} ; j}^{a_{1}^{\prime} a_{2}^{\prime}}\right\rangle e_{a_{1} a_{2} ; i}^{a_{3}} \otimes e_{a_{1}^{\prime} a_{2}^{\prime} ; j}^{a_{3}^{\prime}},
$$

where $e_{a_{1} a_{2} ; i}^{a_{3}}$ and $f_{a_{3}}^{a_{1} a_{2} ; j}$ are basis vectors given in (4.13) and (4.15) and $\langle\cdot, \cdot\rangle$ is a bilinear pairing given by

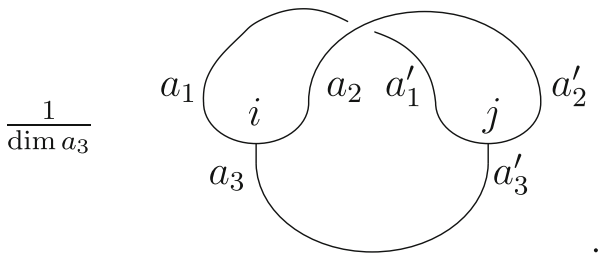

Notice that $V_{c l}^{\prime}$ has the same decomposition as $V_{c l}$ in (5.33). They are isomorphic as $V \otimes V$-modules. There is, however, no canonical isomorphism. Now we choose a particular isomorphism $\varphi_{c l}: V_{c l} \rightarrow V_{c l}^{\prime}$ given by

$$
\varphi_{c l}=\oplus_{a \in \mathcal{I}} \frac{D}{\operatorname{dim} a} e^{-2 \pi i h_{a}} \operatorname{id}_{W_{a} \otimes W_{a}^{\prime}}
$$

The isomorphism $\varphi_{c l}$ induces a nondegenerate invariant bilinear form on $V_{c l}$ viewed as $V \otimes V$-module.

The following theorem is a categorical version of Theorem 5.1 in [HKo3]. We give a categorical proof here.

Theorem 5.17. $\left(V_{c l}, \mu_{c l}, \iota_{c l}\right)$ together with the isomorphism $\varphi_{c l}$ gives a modular invariant commutative Frobenius algebra in $\mathcal{C}_{V \otimes V}$ with a trivial twist.

Proof. It was proved in [Ko1] that $\left(V_{c l}, \mu_{c l}, \iota_{c l}\right)$ together with the isomorphism $\varphi_{c l}$ gives a commutative Frobenius algebra with a trivial twist. It remains to show the modular invariance.

First, the bilinear pairing $\langle\cdot, \cdot\rangle$ given in $(5.35)$ can be naturally extended to a bilinear form, still denoted as $\langle\cdot, \cdot\rangle$, on $\oplus_{a, a_{1} \in \mathcal{I}}\left(W_{a_{1}}, W_{a} \otimes W_{a_{1}}\right)$ as follows:

$$
\left\langle f_{a_{1} ; i}^{a a_{1}}, f_{b_{1} ; j}^{b b_{1}}\right\rangle:=\delta_{a b^{\prime}} \delta_{a_{1} b_{1}^{\prime}}\left\langle f_{a_{1} ; i}^{a a_{1}}, f_{a_{1}^{\prime} ; j}^{a^{\prime} a_{1}^{\prime}}\right\rangle
$$

Then it is easy to see that to prove the modular invariance of $V_{c l}$ is equivalent to prove that the bilinear form $\langle\cdot, \cdot\rangle$ on $\bigoplus_{a, a_{1} \in \mathcal{I}}\left(W_{a_{1}}, W_{a} \otimes W_{a_{1}}\right)$ defined above is invariant under the action of $\left(S^{-1}\right)^{*} \otimes S^{*}$. Clearly, when $b \neq a^{\prime},\left\langle\left(S^{-1}(a)\right)^{*} f_{a_{1} ; i}^{a a_{1}},(S(b))^{*} f_{b_{1} ; j}^{b b_{1}}\right\rangle=0$. When $b=a^{\prime}$, we have (using (4.58), (4.59) and (4.44)) 
$\left\langle\left(S^{-1}(a)\right)^{*} f_{a_{1} ; i}^{a a_{1},}\left(S\left(a^{\prime}\right)\right)^{*} f_{b_{1} ; j}^{a^{\prime} b_{1}}\right\rangle=\sum_{a_{3} \in \mathcal{I}} \frac{\operatorname{dim} a_{3}}{D^{2}}$
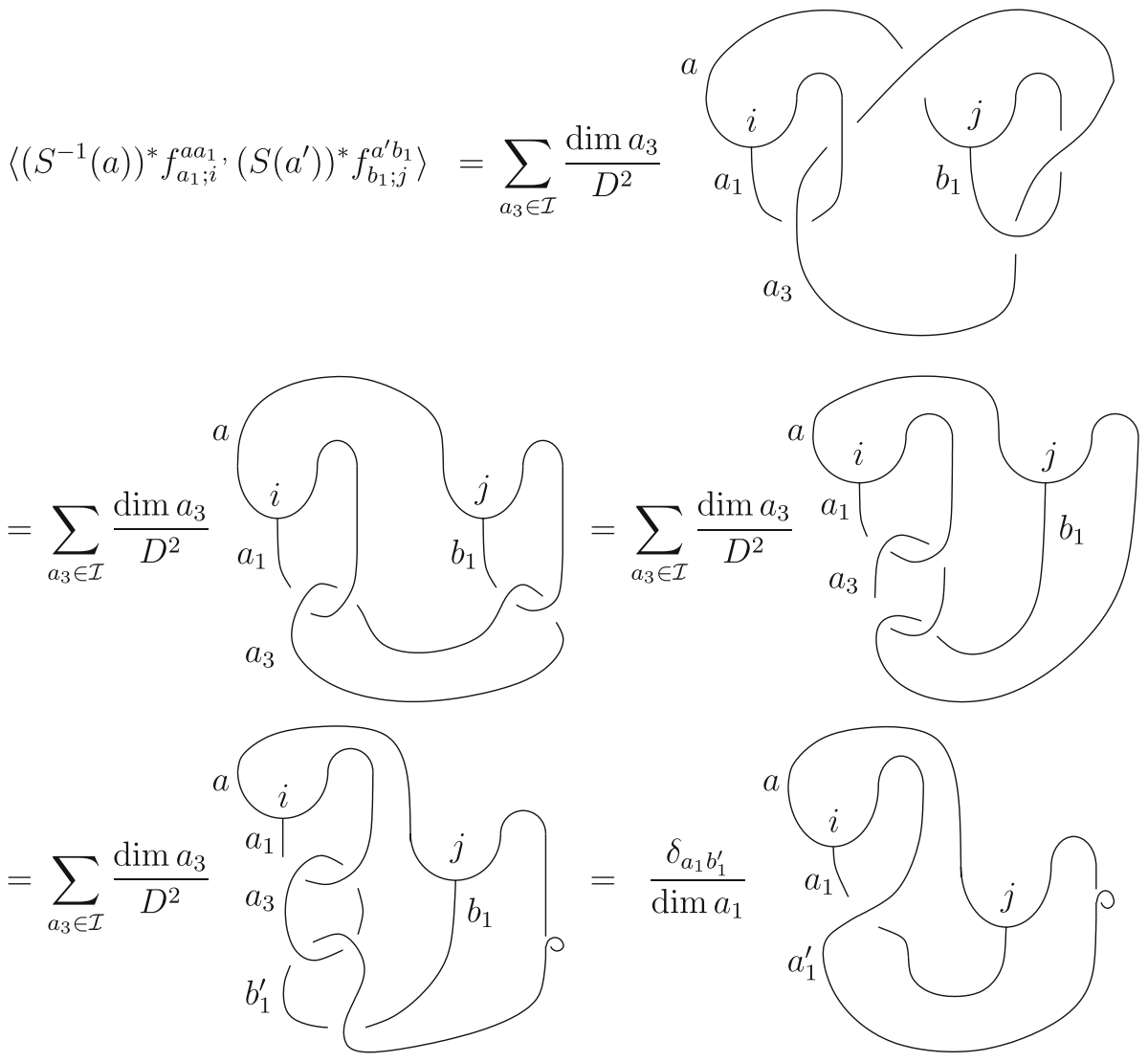

$=\frac{\delta_{a_{1} b_{1}^{\prime}}}{\operatorname{dim} a_{1}}$

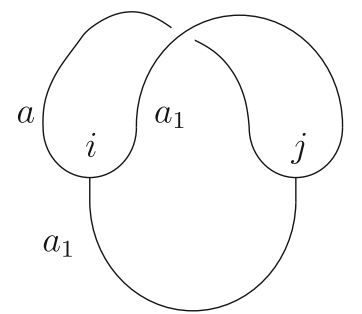

Now we define $V_{o p}$. Let $X$ be a $V$-module. Let $e_{X}: X^{\prime} \otimes X \rightarrow V$ and $i_{X}: V \rightarrow$ $X \otimes X^{\prime}$ be the duality maps defined in [Ko1]. $V_{o p}:=X \otimes X^{\prime}$ has a natural structure of symmetric Frobenius algebra [FS] with $\iota_{o p}:=i_{X}, \mu_{o p}:=\mathrm{id}_{X} \otimes e_{X} \otimes \operatorname{id}_{X^{\prime}}, \epsilon_{o p}:=e_{X^{\prime}}$ and $\Delta_{o p}:=\operatorname{id}_{X} \otimes i_{X} \otimes \operatorname{id}_{X^{\prime}}$.

Now we define a map $\iota_{c l-o p}: T\left(V_{c l}\right) \rightarrow V_{o p}$ by

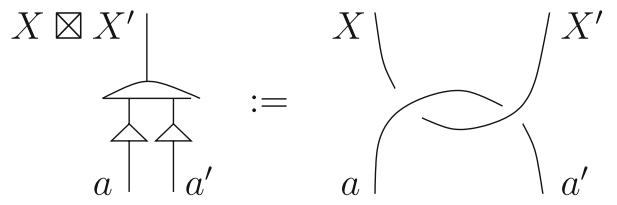


Lemma 5.18.


Proof. By (5.27) and (5.38), we have
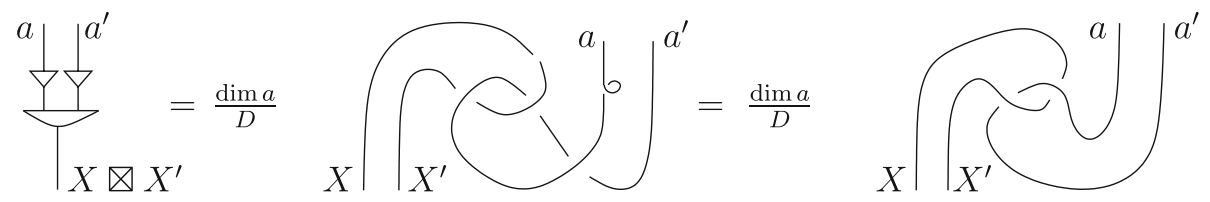

It is easy to see that the last figure in (5.40) can be deformed to that on the right hand side of (5.39).

Theorem 5.19. $\left(V_{c l}, V_{o p}, \iota_{c l-o p}\right)$ is a Cardy $\mathcal{C}_{V} \mid \mathcal{C}_{V \otimes V}$-algebra.

Proof. Recall that $T\left(V_{c l}\right)$ together with the multiplication morphism $\mu_{T\left(V_{c l}\right)}=T\left(\mu_{c l}\right) \circ$ $\varphi_{2}$ and the morphism $\iota_{T\left(V_{c l}\right)}=T\left(\iota_{c l}\right) \circ \varphi_{0}$ is an associative algebra. We first prove that $\iota_{c l-o p}$ is an algebra morphism. It is clear that $\iota_{c l-o p} \circ \iota_{T\left(V_{c l}\right)}=\iota_{o p}$. It remains to show the following identity

$$
\iota_{c l-o p} \circ \mu_{T\left(V_{c l}\right)}=\mu_{o p} \circ\left(\iota_{c l-o p} \bigotimes \iota_{c l-o p}\right) .
$$

By the definition of $\varphi_{2}$, that of $\mu_{c l}$ and (5.38), we obtain

$$
\iota_{c l-o p} \circ \mu_{T\left(V_{c l}\right)}=\sum_{a, b, c \in \mathcal{I}} \sum_{i, j} \frac{1}{\operatorname{dim} c}
$$


It is easy to see that the right hand side of (5.42) equals
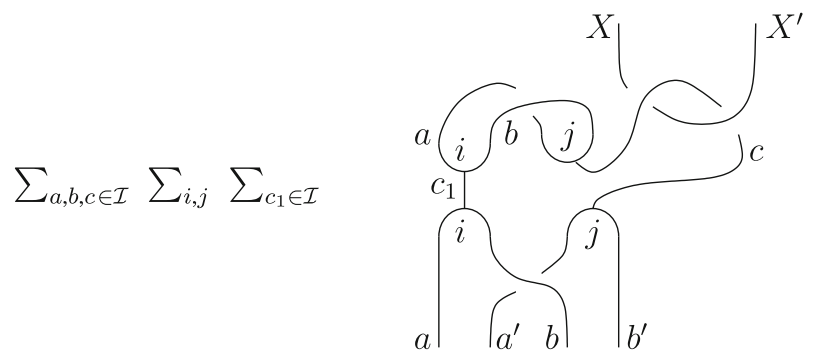
Using (4.17) to sum up the indices $c_{1}$ and $i$, we obtain that (5.43) further equals

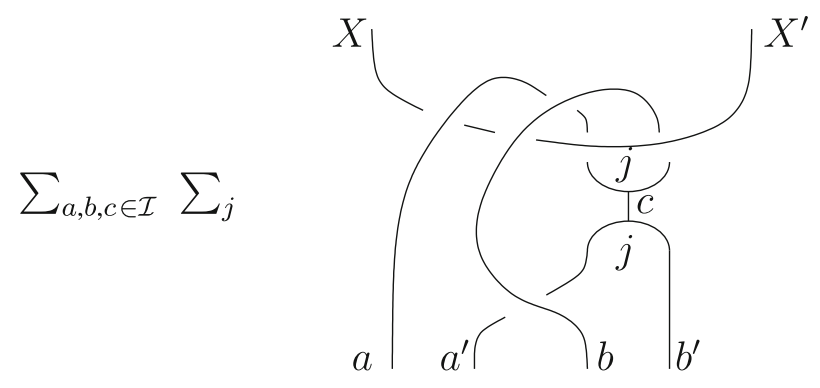

Using (4.17) again, we obtain

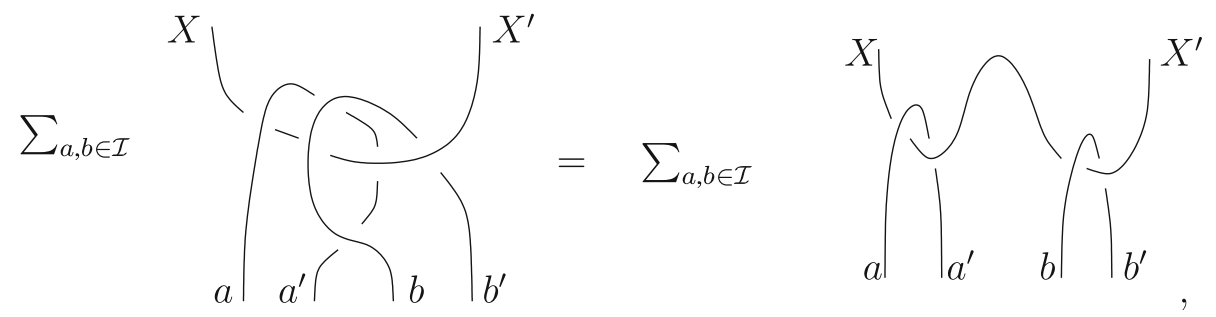

the right hand side of which is nothing but $\mu_{o p} \circ\left(\iota_{c l-o p} \otimes \iota_{c l-o p}\right)$.

The commutativity (5.32) follows from the following identity:

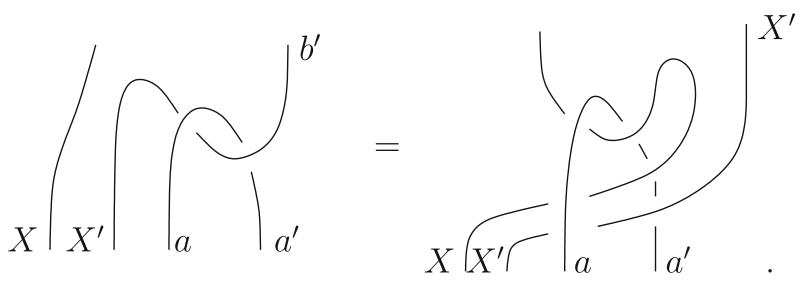

In summary, we have proved that the triple $\left(V_{c l}, V_{o p}, \iota_{c l-o p}\right)$ is an open-closed $\mathcal{C}_{V} \mid \mathcal{C}_{V \otimes V}$-algebra. It remains to show that the Cardy condition (5.30) holds. We use (5.38), (5.39) and the definition of $\mu_{o p}$ and $\Delta_{o p}$ to express both sides of (5.30) graphically. Then it is easy to see that both sides are the deformations of each other.

Theorem 5.19 reflects the general fact that consistent open theories (or D-branes) for a given closed theory are not unique. Instead they form a category. There are many good questions one can ask about Cardy $\mathcal{C}_{V} \mid \mathcal{C}_{V \otimes V}$-algebra, for example its relation to the works of Fuchs, Runkel, Schweigert and Fjelstad [FS,FRS1-FRS4,FjFRS1,FjFRS2]. We leave such topics to $[\mathrm{KR}]$ and future publications.

\section{A. The Proof of Lemma 4.30}

Lemma A.1. For $w_{1} \in W_{a_{1}}, w_{a} \in W_{a_{2}}$ and $w_{a_{3}}^{\prime} \in\left(W_{a_{3}}\right)^{\prime}$ and $\mathcal{Y}_{a_{1} a_{2}}^{a_{3}} \in \mathcal{V}_{a_{1} a_{2}}^{a_{3}}$, we have

$$
\begin{aligned}
& \left\langle w_{a_{3}}^{\prime}, \mathcal{Y}\left(\mathcal{U}(x) w_{a_{1}}, x\right) w_{a_{2}}\right\rangle \\
& =\left\langle\tilde{A}_{r}(\mathcal{Y})\left(\mathcal{U}\left(e^{(2 r+1) \pi i} x^{-1}\right) e^{-(2 r+1) \pi i L(0)} w_{a_{1}}, e^{(2 r+1) \pi i} x^{-1}\right) w_{a_{3}}^{\prime}, w_{a_{2}}\right\rangle
\end{aligned}
$$


Proof. Using the definition of $\hat{A}_{r}$, we see that the left hand side of (A.47) equals

$$
\left\langle\tilde{A}_{r}(\mathcal{Y})\left(e^{-x L(1)} x^{-2 L(0)} \mathcal{U}(x) w_{a_{1}}, e^{(2 r+1) \pi i} x^{-1}\right) w_{a_{3}}^{\prime}, w_{a_{2}}\right\rangle
$$

In [H8], the following formula

$$
e^{x L(1)} x^{-2 L(0)} e^{(2 r+1) \pi i L(0)} \mathcal{U}(x) e^{-(2 r+1) \pi i L(0)}=\mathcal{U}\left(x^{-1}\right)
$$

is proved. Applying (A.49) to (A.48), we obtain (A.47) immediately.

Now we are ready to give a proof of Lemma 4.4 .

Proof. We have, for $w_{a_{2}} \in W_{a_{2}}, w_{a_{3}} \in W_{a_{3}}$,

$$
\begin{aligned}
\left(\left(\Psi_{2}\left(\mathcal{Y}_{a a_{1} ; i}^{a_{1} ;(1)} \otimes \mathcal{Y}_{a_{2} a_{3} ; j}^{a ;(2)}\right)\right)\left(w_{a_{2}} \otimes w_{a_{3}}\right)\right)\left(z_{1}, z_{2}+\tau, \tau\right) \\
=E\left(\operatorname { T r } _ { W _ { a _ { 1 } } } \mathcal { Y } _ { a a _ { 1 } ; i } ^ { a _ { 1 } ; ( 1 ) } \left(\mathcal{U}\left(e^{2 \pi i\left(z_{2}+\tau\right)}\right) .\right.\right. \\
\left.\left.\cdot \mathcal{Y}_{a_{2} a_{3} ; j}^{a ;(2)}\left(w_{a_{2}}, z_{1}-\left(z_{2}+\tau\right)\right) w_{a_{3}}, e^{2 \pi i\left(z_{2}+\tau\right)}\right) q_{\tau}^{L(0)-\frac{c}{24}}\right) \\
=\sum_{b \in \mathcal{I}} \sum_{k, l} F^{-1}\left(\mathcal{Y}_{a a_{1} ; i}^{a_{1} ;(1)} \otimes \mathcal{Y}_{a_{2} a_{3} ; j}^{a ;(2)} ; \mathcal{Y}_{a_{2} b ; k}^{a_{1} ;(3)} \otimes \mathcal{Y}_{a_{3} a_{1} ; l}^{b ;(4)}\right) \\
E\left(\operatorname{Tr}_{W_{a_{1}}} \mathcal{Y}_{a_{2} b ; k}^{a_{1} ;(3)}\left(\mathcal{U}\left(e^{2 \pi i z_{1}}\right) w_{a_{2}}, e^{2 \pi i z_{1}}\right)\right. \\
\left.\quad \mathcal{Y}_{a_{3} a_{1} ; l}^{b ;(4)}\left(\mathcal{U}\left(e^{2 \pi i\left(z_{2}+\tau\right)}\right) w_{a_{3}}, e^{2 \pi i\left(z_{2}+\tau\right)}\right) q_{\tau}^{L(0)-\frac{c}{24}}\right) .
\end{aligned}
$$

Using the $L(0)$-conjugation formula, we can move $q_{\tau}$ from the right side of $\mathcal{Y}_{a_{3} a_{1} ; l}^{b ;(4)}$ to the left side of $\mathcal{Y}_{a_{3} a_{1} ; l}^{b ;(4)}$. Then using the following property of trace:

$$
\operatorname{Tr}_{W_{a_{1}}}(A B)=\operatorname{Tr}_{W_{b}}(B A)
$$

for all $A: W_{b} \rightarrow \overline{W_{a_{1}}}, B: W_{a_{1}} \rightarrow \overline{W_{b}}$ whenever the multiple sums in either side of (A.51) converge absolutely, we obtain that the left hand side of (A.50) equals

$$
\begin{aligned}
& \sum_{b \in \mathcal{I}} \sum_{k, l} F^{-1}\left(\mathcal{Y}_{a a_{1} ; i}^{a_{1} ;(1)} \otimes \mathcal{Y}_{a_{2} a_{3} ; j}^{a ;(2)} ; \mathcal{Y}_{a_{2} b ; k}^{a_{1} ;(3)} \otimes \mathcal{Y}_{a_{3} a_{1} ; l}^{b ;(4)}\right) \\
& \left.E\left(\operatorname{Tr}_{W_{b}} \mathcal{Y}_{a_{3} a_{1} ; l}^{b ;()} \mathcal{U}\left(e^{2 \pi i z_{2}}\right) w_{a_{3}}, e^{2 \pi i z_{2}}\right) \mathcal{Y}_{a_{2} b ; k}^{a_{1} ;(3)}\left(\mathcal{U}\left(e^{2 \pi i z_{1}}\right) w_{a_{2}}, e^{2 \pi i z_{1}}\right) q_{\tau}^{L(0)-\frac{c}{24}}\right)
\end{aligned}
$$

Now apply (A.47) to (A.52). We then obtain that (A.52) equals

$$
\begin{aligned}
\sum_{b \in \mathcal{I}} & \sum_{k, l} F^{-1}\left(\mathcal{Y}_{a a_{1} ; i}^{a_{1} ;(1)} \otimes \mathcal{Y}_{a_{2} a_{3} ; j}^{a ;(2)} ; \mathcal{Y}_{a_{2} b ; k}^{a_{1} ;(3)} \otimes \mathcal{Y}_{a_{3} a_{1} ; l}^{b ;(4)}\right) \\
E & \left(\operatorname{Tr}_{\left(W_{b}\right)^{\prime}} \tilde{A}_{r}\left(\mathcal{Y}_{a_{2} b ; k}^{a_{1} ;(3)}\right)\left(\mathcal{U}\left(e^{(2 r+1) \pi i} e^{-2 \pi i z_{1}}\right) e^{-(2 r+1) \pi i L(0)} w_{a_{2}}, e^{(2 r+1) \pi i} e^{-2 \pi i z_{1}}\right)\right. \\
& \left.\tilde{A}_{r}\left(\mathcal{Y}_{a_{3} a_{1} ; l}^{b ;(4)}\right)\left(\mathcal{U}\left(e^{(2 r+1) \pi i} e^{-2 \pi i z_{2}}\right) e^{-(2 r+1) \pi i L(0)} w_{a_{3}}, e^{(2 r+1) \pi i} e^{-2 \pi i z_{2}}\right) q_{\tau}^{L(0)-\frac{c}{24}}\right) .
\end{aligned}
$$


Now apply the associativity again and be careful about the branch cut as in [H8], then the left hand side of (A.50) further equals to

$$
\begin{aligned}
& \sum_{b \in \mathcal{I}} \sum_{k, l} \sum_{c \in \mathcal{I}} \sum_{p, q} F^{-1}\left(\mathcal{Y}_{a a_{1} ; i}^{a_{1} ;(1)} \otimes \mathcal{Y}_{a_{2} a_{3} ; j}^{a ;(2)} ; \mathcal{Y}_{a_{2} b ; k}^{a_{1} ;(3)} \otimes \mathcal{Y}_{a_{3} a_{1} ; l}^{b ;(4)}\right) \\
& \times F\left(\tilde{A}_{r}\left(\mathcal{Y}_{a_{2} b ; k}^{a_{1} ;(3)}\right) \otimes \tilde{A}_{r}\left(\mathcal{Y}_{a_{3} a_{1} ; l}^{b ;(4)}\right), \mathcal{Y}_{c b^{\prime} ; p}^{b^{\prime} ;(5)} \otimes \mathcal{Y}_{a_{2} a_{3} ; q}^{c ;(6)}\right) \\
& \times E\left(\operatorname{Tr}_{\left(W_{b}\right)^{\prime}} q_{\tau}^{L(0)-\frac{c}{24}} \mathcal{Y}_{c b^{\prime} ; p}^{b^{\prime} ;(5)} \mathcal{U}\left(e^{(2 r+1) \pi i} e^{-2 \pi i z_{2}}\right)\right. \\
& \left.\left.\cdot \mathcal{Y}_{a_{2} a_{3} ; q}^{c ;(6)}\left(e^{-(2 r+1) \pi i L(0)} w_{a_{2}}, e^{\pi i}\left(z_{1}-z_{2}\right)\right) e^{-(2 r+1) \pi i L(0)} w_{a_{3}}, e^{(2 r+1) \pi i} e^{-2 \pi i z_{2}}\right)\right) \text {. } \\
& =\sum_{b \in \mathcal{I}} \sum_{k, l} \sum_{c \in \mathcal{I}} \sum_{p, q} F^{-1}\left(\mathcal{Y}_{a a_{1} ; i}^{a_{1} ;(1)} \otimes \mathcal{Y}_{a_{2} a_{3} ; j}^{a ;(2)} ; \mathcal{Y}_{a_{2} b ; k}^{a_{1} ;(3)} \otimes \mathcal{Y}_{a_{3} a_{1} ; l}^{b ;(4)}\right) \\
& \times F\left(\tilde{A}_{r}\left(\mathcal{Y}_{a_{2} b ; k}^{a_{1} ;(3)}\right) \otimes \tilde{A}_{r}\left(\mathcal{Y}_{a_{3} a_{1} ; l}^{b ;(4)}\right), \mathcal{Y}_{c b^{\prime} ; p}^{b^{\prime} ;(5)} \otimes \mathcal{Y}_{a_{2} a_{3} ; q}^{c ;(6)}\right) \\
& \times E\left(\operatorname { T r } _ { W _ { b } } q _ { \tau } ^ { L ( 0 ) - \frac { c } { 2 4 } } \hat { A } _ { r } ( \mathcal { Y } _ { c b ^ { \prime } ; p } ^ { b ^ { \prime } ; ( 5 ) } ) \left(e^{(2 r+1) \pi i L(0)}\right.\right. \\
& \left.\left.\times \mathcal{Y}_{a_{2} a_{3} ; q}^{c ;(6)}\left(e^{-(2 r+1) \pi i L(0)} w_{a_{2}}, e^{\pi i}\left(z_{1}-z_{2}\right)\right) e^{-(2 r+1) \pi i L(0)} w_{a_{3}}, e^{2 \pi i z_{2}}\right)\right) \text {. }
\end{aligned}
$$

Choosing $r=0$ and using $\mathcal{Y}\left(\cdot, e^{2 \pi i} x\right) \cdot=\Omega_{0}^{2}(\mathcal{Y})(\cdot, x) \cdot$, we obtain

$$
\begin{aligned}
& \left(\left(\Psi_{2}\left(\mathcal{Y}_{a a_{1} ; i}^{a_{1} ;(1)} \otimes \mathcal{Y}_{a_{2} a_{3} ; j}^{a ;(2)}\right)\right)\left(w_{a_{2}} \otimes w_{a_{3}}\right)\right)\left(z_{1}, z_{2}+\tau, \tau\right) \\
& =\sum_{b \in \mathcal{I}} \sum_{k, l} \sum_{c \in \mathcal{I}} \sum_{p, q} F^{-1}\left(\mathcal{Y}_{a a_{1} ; i}^{a_{1} ;(1)} \otimes \mathcal{Y}_{a_{2} a_{3} ; j}^{a ;(2)} ; \mathcal{Y}_{a_{2} b ; k}^{a_{1} ;(3)} \otimes \mathcal{Y}_{a_{3} a_{1} ; l}^{b ;(4)}\right) \\
& \quad \times F\left(\tilde{A}_{0}\left(\mathcal{Y}_{a_{2} b ; k}^{a_{1} ;(3)}\right) \otimes \tilde{A}_{0}\left(\mathcal{Y}_{a_{3} a_{1} ; l}^{b ;(4)}\right), \mathcal{Y}_{c b^{\prime} ; p}^{b^{\prime} ;(5)} \otimes \mathcal{Y}_{a_{2} a_{3} ; q}^{c ;(6)}\right) \\
& \quad \times E\left(\operatorname{Tr}_{W_{b}} \hat{A}_{0}\left(\mathcal{Y}_{c b^{\prime} ; p}^{b^{\prime} ;(5)}\right)\left(\Omega_{0}^{2}\left(\mathcal{Y}_{a_{2} a_{3} ; q}^{c ;(6)}\right)\left(w_{a_{2}}, z_{1}-z_{2}\right) w_{a_{3}}, e^{2 \pi i z_{2}}\right) q_{\tau}^{L(0)-\frac{c}{24}}\right) .
\end{aligned}
$$

By the linear independency proved in [H10] of the last factor in each term of the above sum, it is clear that $\beta$ induces a map given by (4.30).

Acknowledgement. The results in Sect. 2.4 and 3.1 are included in author's thesis. I want to thank my advisor Yi-Zhi Huang for introducing me to this interesting field and for his constant support and many important suggestions for improvement. I thank C. Schweigert for telling me the meaning of boundary states from a physical point of view. I also want to thank I. Frenkel, J. Fuchs, A. Kirillov, Jr. and C. Schweigert for some inspiring conversations on the subject of Sect. 4.2.

\section{References}

[AN] Alexeevski, A., Natanzon, S.M.: Noncommutative two-dimensional topological field theories and hurwitz numbers for real algebraic curves. Sel. Math. 12(3-4), 377-397 (2006)

[BHL] Barron, K., Huang, Y.-Z., Lepowsky, J.: Factorization of formal exponential and uniformization. J. Alg. 228, 551-579 (2000)

[BK1] Bakalov, B., Kirillov, Jr., A.: On the lego-teichmüller game. Transform. Groups 5, 207 (2000)

[BK2] Bakalov, B., Kirillov, Jr., A.: Lectures on Tensor Categories and Modular Functors. University Lecture Series, Vol. 21, Providence, RI: Amer. Math. Soc., 2001

[C1] Cardy, J.L.: Conformal invariance and surface critical behavior. Nucl. Phys. B 240, 514-532 (1984) 
[C2] Cardy, J.L.: Effect of boundary conditions on the operator content of two-dimensional conformally invariant theories. Nucl. Phys. B275, 200-218 (1986)

[C3] Cardy, J.L.: Operator content of two-dimensional conformal invariant theories. Nucl. Phys. B270, 186-204 (1986)

[C4] Cardy, J.L.: Boundary conditions, fusion rules and the verlinde formula. Nucl. Phys. B324, 581-596 (1989)

[DLM] Dong, C.-Y., Li, H.-S., Mason, G.: Modular-invariance of trace functions in orbifold theory and generalized moonshine. Commun. Math. Phys. 214, 1-56 (2000)

[FFFS1] Felder, G., Fröhlich, J., Fuchs, J., Schweigert, C.: The geometry of wzw branes. J. Geom. Phys. 34, 162-190 (2000)

[FFFS2] Felder, G., Fröhlich, J., Fuchs, J., Schweigert, C.: Correlation functions and boundary conditions in rational conformal field theory and three-dimensional topology. Compositio Math. 131, 189-237 (2002)

[FFRS] Fröhlich, J., Fuchs, J., Runkel, I., Schweigert, C.: Correspondences of ribbon categories. Adv. Math. 199(1), 192-329 (2006)

[FjFRS1] Fjelstad, J., Fuchs, J., Runkel, I., Schweigert, C.: Tft construction of rcft correlators v: proof of modular invariance and factorisation. Theory and Appl. of Categ 16, 392-433 (2006)

[FjFRS2] Fjelstad, J., Fuchs, J., Runkel, I., Schweigert, C.: Uniqueness of open/closed rational CFT with given algebra of open states. http://arXiv.org/listhep-th/0612306, 2006

[FHL] Frenkel, I.B., Huang, Y.-Z., Lepowsky, J.: On axiomatic approaches to vertex operator algebras and modules. Memoirs Amer. Math. Soc. 104, 1993

[FRS1] Fuchs, J., Runkel, I., Schweigert, C.: Conformal correlation functions, frobenius algebras and triangulations. Nucl. Phys. B624, 452-468 (2002)

[FRS2] Fuchs, J., Runkel, I., Schweigert, C.: Tft construction of rcft correlators I: partition functions. Nucl. Phys. B678, 511 (2004)

[FRS3] Fuchs, J., Runkel, I., Schweigert, C.: Tft construction of rcft correlators III: simple currents. Nucl. Phys. B694, 277 (2004)

[FRS4] Fuchs, J., Runkel, I., Schweigert, C.: Tft construction of rcft correlators IV: structure constants and correlation functions. Nucl. Phys. B 715(3), 539-638 (2005)

[FS] Fuchs, J., Schweigert, C.: Category theory for conformal boundary conditions. Fields Institute Commun. 39, 25 (2003)

[G] Gan, W.L.: Koszul duality for dioperads. Math. Res. Lett. 10(1), 109-124 (2003)

[H1] Huang, Y.-Z.: Geometric interpretation of vertex operator algebras. Proc. Natl. Acad. Sci. USA 88, 9964-9968 (1991)

[H2] Huang, Y.-Z.: A theory of tensor products for module categories for a vertex operator algebra, iv. J. Pure Appl. Alg. 100, 173-216 (1995)

[H3] Huang, Y.-Z.: Two-dimensional conformal geometry and vertex operator algebras. Progress in Mathematics, Vol. 148, Boston: Birkhäuser, 1997

[H4] Huang, Y.-Z.: Generalized rationality and a "jacobi identity" for intertwining operator algebras. Selecta Math. (N. S.) 6, 225-267 (2000)

[H5] Huang, Y.-Z.: A functional-analytic theory of vertex (operator) algebras. I. Commun. Math. Phys. 204(1), 61-84 (1999)

[H6] Huang, Y.-Z.: A functional-analytic theory of vertex (operator) algebras. II. Commun. Math. Phys. 242(3), 425-444 (2003)

[H7] Huang, Y.-Z.: Differential equations and intertwining operators. Commun. Contemp. Math. 7, 375-400 (2005)

[H8] Huang, Y.-Z.: Riemann surfaces with boundaries and the theory of vertex operator algebras. Fields Institute Commun. 39, 109 (2003)

[H9] Huang, Y.-Z.: Differential equations, duality and modular invariance. Commun. Contemp. Math. 7, 649-706 (2005)

[H10] Huang, Y.-Z.: Vertex operator algebras and the verlinde conjecture. Commun. Contemp. Math. 10(1), 103-154 (2008)

[H11] Huang, Y.-Z.: Rigidity and modularity of vertex tensor categories. math.QA/0502533, 2005

[HKo1] Huang, Y.-Z., Kong, L.: Open-string vertex algebra, category and operad. Commun. Math. Phys. 250, 433-471 (2004)

[HKo2] Huang, Y.-Z., Kong, L.: Full field algebras. Commun. Math. Phys. 272, 345-396 (2007)

[HKo3] Huang, Y.-Z., Kong, L.: Modular invariance for conformal full field algebras, math.QA/0609570, 2006

[HL1] Huang, Y.-Z., Lepowsky, J.: Tensor products of modules for a vertex operator algebra and vertex tensor categories, In: Lie Theory and Geometry, in honor of Bertram Kostant, R. Brylinski, J.-L. Brylinski, V. Guillemin, V. Kac (Eds.) Boston: Birkhäuser, 1994, pp. 349-383 
[HL2] Huang, Y.-Z., Lepowsky, J.: A theory of tensor products for module categories for a vertex operator algebra, I. Selecta Math. (N.S.) 1, 699-756 (1995)

[HL3] Huang, Y.-Z., Lepowsky, J.: A theory of tensor products for module categories for a vertex operator algebra, II. Selecta Math. (N.S.) 1, 757-786 (1995)

[HL4] Huang, Y.-Z., Lepowsky, J.: A theory of tensor products for module categories for a vertex operator algebra, III. J. Pure Appl. Alg. 100, 141-171 (1995)

[I] Ishibashi, N.: The boundary and crosscap states in conformal field theories. Mod. Phys. Lett. A4, 251 (1989)

[Ko1] Kong, L.: Full field algebras, operads and tensor categories. Adv. Math. 213, 271-340 (2007)

[Ko2] Kong, L.: Open-closed field algebras. Commun. Math. Phys. 280, 207-261 (2008)

[Kont] Kontsevich, M.: Operads and motives in deformation quantization. Lett. Math. Phys. 48, 35-72 (1999)

[Ki] Kirillov,, Jr. A.: On an inner product in modular tensor categories. J. Amer. Math. Soc 9(4), 1135-1169 (1996)

[Kr] Kriz, I.: On spin and modularity of conformal field theory. Ann. Sci. École Norm. Sup. (4) 36(1), 57-112 (2003)

[KR] Kong, L., Runkel, I.: Cardy algebras and sewing constraints, I, II, in preparation

[La] Lazaroiu, C.I.: On the structure of open-closed topological field theory in two dimensions. Nucl. Phys. B603, 497-530 (2001)

[Le] Lewellen, D.C.: Sewing constraints for conformal field theories on surfaces with boundaries. Nucl. Phys. B372, 654 (1992)

[Ly] Lyubashenko, V.: Modular transformations for tensor categories. J. Pure Appl. Alg. 98(3), 297-327 (1995)

[LL] Lepowsky, J., Li, H.-S.: Introduction to vertex operator algebras and their representations. Progress in Mathematics, 227, Boston, MA: Birkhäuser Boston, Inc. 2004

[LP] Lauda, A., Pfeiffer, H.: Open-closed strings: two-dimensional extended tqfts and frobenius algebras. Topology Appl 155(7), 623-666 (2008)

[Mi1] Miyamoto, M.: Modular invariance of vertex operator algebras satisfying $c_{2}$-cofiniteness. Duke Math. J. 122(1), 51-91 (2004)

[Mi2] Miyamoto, M.: Intertwining operators and modular invariance. math.QA/0010180, 2000

[Mo1] Moore, G.: Some comments on branes, $g$-flux, and $k$-theory. Internat. J. Mod. Phys. A16, 936-944 (2001)

[Mo2] Moore, G.: D-branes, RR-Fields and K-Theory, I, II, III, VI. Lecture notes for the ITP miniprogram: The duality workshop: a Math/Physics collaboration, June, 2001; http://online.itp.ucsb. edu/online/mp01/moore1

[MSei1] Moore, G., Seiberg, N.: Classical and quantum conformal field theory. Commun. Math. Phys. 123, 177-254 (1989)

[MSei2] Moore, G., Seiberg, N.: Naturality in conformal field theory. Nucl. Phys. B313, 16-40 (1989)

[MSei3] Moore, G., Seiberg, N.: Lecture on RCFT. In: Physics Geometry and Topology. Edited by H.C. Lee, New York: Plenum Press, 1990

[MSeg] Moore, G., Segal, G.: D-branes and K-theory in 2D topological field theory. hep-th/0609042, 2006

[RT] Reshetikhin, N., Turaev, V.G.: Invariants of 3-manifolds via link polynomials of quantum groups. Invent. Math. 103(3), 547-597 (1991)

[Se1] Segal, G.: The definition of conformal field theory, Preprint, 1988; also In: Topology, geometry and quantum field theory. ed. U. Tillmann, London Math. Soc. Lect. Note Ser., Vol. 308. Cambridge: Cambridge University Press, 2004, 421-577

[Se2] Segal, G.: Topological structures in string theory. R. Soc. Lond. Philos. Trans. A359, 1389-1398 (2001)

[So] Sonoda, H.: Sewing conformal field theories, I, II. Nucl. Phys. B311, 401-416, 417-432 (1988)

[T] Turaev.: Quantum invariant of knots and 3-manifolds, de Gruyter Studies in Mathematics, Vol. 18, Berlin: Walter de Gruyter, 1994

[V] Voronov, A.A.: The Swiss-cheese operad. In: Homotopy invariant algebraic structures, in honor of J. Michael Boardman, Proc. of the AMS Special Session on Homotopy Theory, Baltimore, 1998, ed. J.-P. Meyer, J. Morava, W. S. Wilson, Contemporary Math., Vol. 239, pp. 365-373 Amer. Math. Soc., Providence, RI, 1999

[Z] Zhu, Y.-C.: Modular invariance of vertex operator algebras. J. Amer. Math. Soc. 9, 237-302 (1996) 